\title{
Accurate positions and finding charts of known planetary nebulae in the Large Magellanic Cloud ${ }^{\star, \star \star, \star \star \star}$
}

\author{
P. Leisy ${ }^{1}$, M. Dennefeld ${ }^{1,2}$, C. Alard ${ }^{3}$, and J. Guibert ${ }^{3}$ \\ 1 Institut d'Astrophysique de Paris 98bis, B ${ }^{\mathrm{d}}$ Arago 75014 Paris, France \\ 2 Université Pierre et Marie Curie, Paris, France \\ 3 Centre d'Analyse des Images and Observatoire de Paris, DEMIRM/URA 337 du CNRS, 77 Avenue Denfert Rochereau 75014 \\ Paris, France
}

Received July 28, 1995; accepted June 10, 1996

\begin{abstract}
We present identifications and accurate positions for about 280 planetary nebulae $(\mathrm{PNe})$ in the Large Magellanic Cloud (LMC). These positions are needed for follow-up ground or space observations. Entire Schmidt plates have been scanned with the MAMA microdensitometer, and PPM stars used for the astrometric reduction. The precision achieved is always better than $0 . \prime 5$, and better than 0.13 for most of the objects not located close to the border of the plates. Photometry in the $B$ band is provided with an accuracy of about 0.2 magnitude, except in too crowded zones where the background cannot be determined. Cross-identifications with IRAS sources have also been tabulated and discussed.
\end{abstract}

Key words: planetary nebulae: general - galaxies: Magellanic Clouds - galaxies: ISM; stellar content astrometry

\section{Introduction}

The study of physical properties of planetary nebulae $(\mathrm{PNe})$, and , in particular, the determination of their luminosity function is hampered in our Galaxy by the poor knowledge of their distances. This problem can be overcome by going to external galaxies, like the Magellanic Clouds, where the distance is known with a reasonable accuracy and where, in first approximation, all PNe can be taken to be located at the same distance. Several photographic surveys, either by direct imaging or through objective-prism, have been devoted in the past to the

Send offprint requests to: $\mathrm{P}$. Leisy

* Plate scanning done with MAMA, Paris Observatory.

** Based on observations made at the European Southern Observatory, La Silla, Chile.

${ }^{\star \star \star}$ Tables also available in electronic form at CDS. search of emission-line objects in the Clouds, but their limited sensitivity did not allow the detection of the faintest objects even at these nearby distances. Progress in detector technologies however open the prospect to get complete samples of such objects in Local Group Galaxies in the near future.

The possibilities of photographic plates can however still be further exploited in the meantime. Sky Survey Schmidt plates are ideal tools to measure a large number of objects spread over a large area like the Magellanic Clouds. They allow easily a determination of positions with an accuracy better than $0.5^{\prime \prime}$, a significant improvement over available coordinates. Such accurate coordinates are nowadays indispensable for follow-up work, with ground-based facilities like multi-object spectrographs or for centering with space instruments. They are further necessary for cross-identification with other surveys, devoted specifically to $\mathrm{PNe}$ or more general ones, like the Deep European Near IR Sky Survey (DENIS). At the same time, these Schmidt plates provide finding charts still necessary for detailed study in crowded regions like the Bar, charts often missing in earlier surveys. Finally, reasonable magnitudes (to $0.1-0.2 \mathrm{~m}$ accuracy) can be provided in an homogeneous set for a large sample, allowing a first sight at the luminosity function.

We have therefore embarked on a program of systematic measure of positions and magnitudes of the known PNe in both Clouds by digitizing Survey Plates (blue and red) over the whole Cloud area, with pixels of 10 microns (or 0"'67). This work had to be supplemented by CCD observations, to recover some of the cataloged, but unidentified PNe and provide photometric zero points.

This first paper describes the methods and first results in the Large Magellanic Cloud. Section 2 describes the main PNe catalogues used as input. We explain the observations and reduction techniques in Sect. 3, while 
Sect. 4 gives the results. We finally work out, as an example of possibilities opened by these results, the crossidentifications with IRAS data in the last section.

\section{Existing surveys, confirmed and rejected candidates}

\subsection{First surveys}

Early work on $\mathrm{H} \alpha$ emission-line stars and nebulae was published by Henize in 1956. This survey, based on objective-prism photographs taken with a 10-inch camera, yielded 415 objects in the LMC, and among them 97 point-like emission sources, with no visible continuum, which could thus be considered as candidates PNe. Finding charts were provided. Lindsay \& Mullan (1963) and Lindsay (1963), using the same instrument, selected, out of a sample of about 1000 emission objects, 109 pointlike $\mathrm{H} \alpha$ emission sources with no continuum. 65 of these were classified as candidate PN because emission lines other than $\mathrm{H} \alpha$ were also seen, while the remaining $44 \mathrm{ob}-$ jects showed only $\mathrm{H} \alpha$. This later group however potentially also contained PNe, because it consisted of fainter objects where other emission lines needed more sensitive material to be revealed. Unfortunately, no finding charts were ever published for this sample, so that recovery of all the PNe is necessary.

In 1964, Westerlund and Smith used a blue objectiveprism with a 20/26 inch Schmidt telescope to survey 100 square degrees of the LMC and produced a catalogue of 42 LMC PNe with approximate coordinates and manually drawn finding charts. Only point sources were retained.

\subsection{Sanduleak et al. (1978), (1984)}

Sanduleak et al. (1978) obtained objective prism plates with the Curtis Schmidt telescope at Cerro Tololo over a large fraction of the LMC. They presented a list (SMP) of $102 \mathrm{PNe}$, with cross-references to previous work. Objects were selected with no evidence of a continuum, and simultaneously with unresolved structure on direct plates (with the possible exception of SMP007). Unfortunately the coordinates are only approximate and no finding charts are provided, which makes the identification difficult. For further studies, these PNe have therefore to be "re-discovered". This was the most complete list of confirmed PNe at that time and therefore their designation is preferred to older ones. Some objects, believed to be of to low excitation to represent classical PNe (and denominated Very Low Excitation (VLE) objects) were presented in an independent list (Sanduleak \& Philipp 1977). A few of the objects initially rejected by SMP, have been detected again in later surveys.

Sanduleak (1984) added $26 \mathrm{PNe}$ (denoted Sa in our table, from Sa104 to Sa126) candidates to this list thanks to deeper plates obtained in the central region of the LMC.
Nine of those had already been found by Jacoby (1980), but have been labeled with the Sanduleak denomination in our table.

Five objects were later rejected. The candidate Sa103 is a galaxy, with $z=0.035$, see Dennefeld (1986). Four objects (108-119-125-127) could not be found again: we took $\mathrm{ON}$ and OFF line CCD images in $\mathrm{H} \alpha$ and couldn't detect any emission object inside the 4 fields (from $3.2^{\prime} \times 2.5^{\prime}$ for Sa108 and Sa119, to $4.3^{\prime} \times 3^{\prime}$ for Sa125, and up to $7^{\prime} \times 7^{\prime}$ for Sa127).

We note that almost all the objects observed with the smaller field (0.90 m Dutch telescope + CCD camera, with a field of $2.5^{\prime} \times 3.2^{\prime}$ ) fall on the edges of the field. As an example, Sa104 was hard to recovered because the position was erroneous by about $2^{\prime}$. However for Sa125 and Sa127 the field is large enough to conclude that no PNe are present in these 2 regions. This has been independently confirmed by Morgan \& Good (1992) who suggest in addition that Sa108 and Sa119 are carbon stars.

Sa104a was first discovered by Savage et al. (1982) and is a true PNe (with particularly low abundances, Leisy \& Dennefeld 1995).

Later on, while this work was in progress, Meatheringham et al. (1991a,b) published, with their spectroscopic observations, better positions for 60 out of the 102 SMP PNe, while 10 others were measured by Vassiliadis et al. (1992). However the R.A. of these 70 $\mathrm{PNe}$ are rounded-up to the nearest second of time!

\subsection{Jacoby (1980)}

Jacoby (1980) used the Cerro Tololo $4 \mathrm{~m}$ telescope at the prime focus with a direct imaging technique (on-line/offline narrow filters photography at [O III] and $\mathrm{H} \alpha$ ) in 4 central regions of LMC (small area of the Bar). This is a much deeper survey which produced 41 faint $\mathrm{PNe}, 5-6$ mag fainter than the brightest ones. Later on, Boroson \& Liebert (1989) rejected 14 objects: 9 were early type stars, $3 \mathrm{M}$ giant stars, 2 objects were not found at all. Furthermore, 8 PNe candidates were already known and confirmed by previous surveys. The coordinates are good to $2^{\prime \prime}$ but the finding charts are difficult to use at the telescope because they are taken in narrow-band emission line filters: the object is then difficult to recognize in a typical telescope acquisition camera or a broad band CCD image.

In 1990, Jacoby et al. published the [OIII] photometry and interference filter images of the fields of the $102 \mathrm{PNe}$ from the first Sanduleak list (1978). These images suffer from the same shortcomings at the telescope as only the brightest stars are visible. 


\subsection{Morgan and Good (1992), (1994)}

Recently Morgan \& Good (1992) and Morgan (1994) published respectively 86 and 54, new PNe candidates from plates taken with the $1.2 \mathrm{~m}$ UKST and objective prism, covering the entire LMC system. The errors on coordinates are "typically $\pm 2-3$ arcsec". Finding charts are provided, but the first series is hardly usable, specially in crowded areas, because of the small dynamics. These objects have also been included here for completeness and homogeneity.

The various lists discussed above provide the basic set of candidate PNe used here. No attempt has been made to be exhaustive. It should be further noted that these are only candidates, and only follow-up slit spectroscopy can confirm the nature of the object. When this was available (from our or published work), it is noted in Table 4 . To date, 139 only out of the 277 candidates have been observed with slit spectroscopy.

\section{Observations and reductions}

To obtain identifications and accurate coordinates, two types of follow-up observations were obtained. For those objects were no finding chart was available at the time, CCD images were obtained with interference filters centered $\mathrm{ON}$ and $\mathrm{OFF}$ the $\mathrm{H} \alpha$ emission line, the images being centered on the published coordinates (see Sect. 3.1). This allowed to recover one (or more) emission-line objects in the field. For other PNe, the identification was taken from the literature. For all the identified $\mathrm{PNe}$ candidates, entire Schmidt plates were scanned, covering the whole LMC, to determine accurate coordinates and a rough broad-band magnitude.

\section{1. $C C D$ imaging}

Images of about $50 \mathrm{PN}$ candidates have been taken, from 1990 to 1991, with several telescopes in ESO La Silla (NTT, $2.2 \mathrm{~m}$ and the Dutch $90 \mathrm{~cm}$ ) with respective fields of view of $7^{\prime} \times 7^{\prime}, 4.3^{\prime} \times 3^{\prime}$ and $3.2^{\prime} \times 2.5^{\prime}$. A few objects turned out to be resolved in the $\mathrm{H} \alpha$ images (SMP011, SMP017, SMP024, SMP030, SMP060, SMP086, J26). All candidates in these fields could be recovered, with the exception of Sa108, Sa119, Sa125 and Sa127 which are therefore rejected as PNe candidates (see Sect. 2.2). Many broad band images were also recorded, just before spectroscopy, to allow a proper positioning of the objects on the slit. These images also provide high quality finding charts.

\subsection{Scanning of Schmidt plates}

We have scanned 5 SERC J (Blue) and 3 ESO (Red) Schmidt plates (to cover a maximum area of the LMC) with the MAMA (Machine à Mesurer Automatique) at
Paris Observatory. MAMA is a linear scanner of 1024 diodes mounted on a stable marble table in a cooled room and provides excellent stability and accuracy (see Berger et al. 1991). The dynamics is 12 bits, therefore with very deep exposures all the stars of $M_{B}=16-17$ or brighter are already saturated. For stars brighter than $M_{B}=12$ the saturation is too strong, and then no precise centering can be done. This dynamics is however perfectly adequate for copy Survey Plates such as the ones we used, where the intrinsic dynamics is anyway limited. The $\mathrm{J}$ plates, with a field about $6^{\circ}$ by $6^{\circ}$, are mapped in a mosaic of 33 by $33,1024 \times 1024$ pixel images, while the mosaic is 28 by 28 in the case of the red plates. The total $\mathrm{J}$ image is $33792 \times 33792$ pixels and occupies about 2.2 Gbytes of disk space. The pixel size is $10 \mu \mathrm{m}$ or $00^{\prime \prime} 67$. The main characteristics of these plates are shown in Table 1 . The coordinates are the $(\alpha, \delta)$ of the plate center in J2000 equinox derived from our astrometry. The date of observation is also given. The last column presents respectively the initial and the final number of PPM (Röser \& Bastian 1988) stars taken into account for the astrometric reduction (see below).

Table 1. Parameters of the used Schmidt plates

\begin{tabular}{|c|c|c|c|c|}
\hline \multirow[t]{2}{*}{ Plate field } & \multicolumn{2}{|c|}{ Plate center J2000 } & \multirow{2}{*}{ Epoch } & \multirow{2}{*}{ PPM } \\
\hline & R.A. & DEC. & & \\
\hline SERC 033J & 52826.94 & -745745.8 & 1976.010 & $278-263$ \\
\hline ESO & 63356.87 & -750231.2 & & 203-195 \\
\hline ESC & 41 & -694756.1 & & \\
\hline SER & 51134 & -695630.7 & & $235-226$ \\
\hline SERC 057J & 60330.15 & -700016.4 & 1975.936 & $248-232$ \\
\hline SERC & $\begin{array}{lll}508 & 14.28\end{array}$ & -645615.1 & 1976.925 & $276-265$ \\
\hline SERC 086J & 55210.55 & -645925.4 & 197 & $255-246$ \\
\hline ESO 087R & 63617.86 & -650437.6 & 1982.068 & $191-171$ \\
\hline
\end{tabular}

\section{3. positions}

The published coordinates for the various $\mathrm{PNe}$ candidates are given in several equinoxes (1950, 1975 and 2000). Most of the astronomical reductions have probably been done (although not explicitly said, except by Jacoby 1980) with earlier catalogues in the FK4 system, whose accuracy of positions and proper motions are no longer satisfactory. Therefore, in order to make the comparison with our new positions, we converted all the coordinates to the J2000 equinox with the matrix suggested by Lieske (1979).

On each SERC or ESO plate, all the PPM references (in equinox J2000 and in the FK5 system) stars have been searched (the mean number is about 200), and their positions on the plates determined in $(X, Y)$. For all the (bright) PPM stars we used a spike centering method. 
The Schmidt plate is then mapped with a bidimensional 3 -degree polynomial in order to transform the $(X, Y)$ to $(\alpha, \delta)$ or to make the reverse transformation. The program is iterative, in the sense that it rejects the reference stars which deviate by more that $3 \sigma$ from the calculated position and recalculates the new coefficients of the fit. On the average, the program iterates only $3-4$ times and rejects only about 10 stars randomly positioned on the plate (and not at the outer part as would be the case for a bad fitting on the borders).

Fig. 1. Differences between our new positions and previously published ones (new-old)

Figure 1 shows the differences between our new positions and the ones given in previous papers. In the case of Sanduleak et al. (1978) objects the original coordinates have been taken only if better one's were not available. The difference can be as large as $2^{\prime}$ and therefore the identification is made impossible in such dense fields from coordinates alone.

We used the same symbols for Figs. 1, 2 and 3: squares (Sanduleak et al. 1978, 1984; and Meatheringham et al. 1991a,b); crosses (Jacoby 1980); circles (Morgan \& Good 1992; Morgan 1994). Figure 2 is an enlargement of the central part of Fig. 1 and shows only the points with better original coordinates (Jacoby 1980; Morgan \& Good 1992; Morgan 1994; and Meatheringham et al. 1991a,b). The accuracy is still not satisfactory. Moreover we see a systematic difference in coordinates, both in $\alpha$ and $\delta$, of respectively $1^{\prime \prime}$ and $2^{\prime \prime}$. The bulk of data is not centered at the origin indicated by the cross.
Fig. 2. Enlargement of the previous figure. Systematic difference in positions (new-old) of about $1^{\prime \prime}$ is clearly visible. The cross indicates the center at position 0,0

The origin of this discrepancy is probably multiple. It could be due to the use of film copies for some earlier measurements, to systematic differences between astrometric catalogues (PPM versus SAO, Perth70, ...), to differences between reference systems (FK5 versus FK4, amounting to about -0. .' 5 in $\alpha$ and +0 .' 2 in $\delta$ (Schwan 1988)), to unaccounted proper motions of standard stars, etc ... The measurements presented here are however based on the best available material (glass copies, digitized images, PPM stars).

The $(X, Y)$ positions of all objects have been measured with the 2 completely different methods. The first method is a Gaussian centering fit inside the MIDAS Image Processing Package of ESO. In very crowded fields, like the Bar, and/or with tightly blended stars, the fit does not converge properly and this measurement has not been retained.

The second is a home-made program (Alard 1995) which separates the blended stars, measures the central position and does the photometry of all the stars found in the field. This method failed only in a few peculiar cases were no position and photometry could be derived $(7$ objects in total from the BAR: SMP047, SMP048, J12, J22, J26, Mo15 and Mo23). These cases are indicated by a colon in Table 4 (Col. 3 after the flag number).

Figure 3 shows that for all the objects, which could be measured by the two methods, the two determinations agree quite well. They gave similar results with a difference always less than $00^{\prime \prime} 30$ and a $\sigma$ of about $00^{\prime \prime} 09$. Both methods 
To look at the internal consistency, we use PNe in common on all plate pairs. The mean position error of the PPM stars at the LMC declination is $00^{\prime \prime} 11$ and the estimated residual systematic deviation between PPM south and the FK5 system has a typical size of $0{ }^{\prime \prime} 05$.

The external consistency has been checked over all overlapping PPM stars on each pair of plates, and no systematic error could be found nor border fitting problems. The residual errors are of the order of $0^{\prime \prime} 2$. Figure 4 shows the results of this test for the plate pair SERC 056J/SERC 085J.

Table 2 lists the number of $\mathrm{PNe}$ in common, as well as the corresponding sigma in coordinate differences. This test was done on the SERC plates only (overlap on ESO

Fig. 3. Half difference between the two centering methods. No systematic effect can be found

have advantages and disadvantages. The first one, with manual identification, is indispensable when an object has poor coordinates but can be identified on a finding-chart. Its limits are reached in crowded fields not because of identification problems, but because the centering algorithm in MIDAS is not able to properly distinguish between sky background and adjacent objects. The second, automatic method is optimized for these difficult cases but, because no a-priori identification is made, the PN candidate cannot be selected out from the outputs when its initial coordinates are too poorly determined. Both methods are thus complementary and have been used in sequence.

We first identified the candidate, to center it in an extracted sub-frame of $128 \times 128$ pixel (in order to produce the finding-charts). We then use first and second methods in this sub-frame only to determine the position and the magnitude of the PNe. In all the cases where the first method works properly, the two $(X, Y)$ position determinations agree perfectly. Therefore, in order to improve the accuracy and to reduce dispersion, the two determinations have been simply averaged. The final PNe coordinates are directly determined from the proper $(X, Y)$ to $(\alpha, \delta)$ transformation, and averaged if several positions were measured on different Schmidt plates. In the case of multiple determinations, if one is coming from a plate corner, check of the reliability is done automatically and the point is rejected if its distance to the mean value is greater than $0^{\prime \prime} 30$ (see the final position Table 4 in Appendix). The definition of a corner is the area within $4 \mathrm{~cm}$, in both directions, of the scanned border zone. Elsewhere, no distortion problem has been noted.
Fig. 4. PPM position differences on Plates SERC 056J and SERC 085J

Table 2. PNe in common

\begin{tabular}{|l|r|r|}
\hline Plates & PNe & $\sigma$ \\
\hline $56-57$ & 64 & $0^{\prime \prime} 16$ \\
$56-85$ & 35 & $0^{\prime \prime} 11$ \\
$56-86$ & 17 & $0^{\prime \prime} 25$ \\
$56-33$ & 8 & $0^{\prime \prime} 16$ \\
$57-85$ & 6 & $0^{\prime \prime} 16$ \\
$57-86$ & 15 & $0^{\prime \prime} 11$ \\
$57-33$ & 8 & $0^{\prime \prime} 14$ \\
$85-86$ & 27 & $0^{\prime \prime} 20$ \\
\hline
\end{tabular}


plates is too small) but ensures the accuracy and consistency of the whole astrometry. Figure 5 represents the histogram of coordinates differences for all the overlapping $\mathrm{PNe}$ on the 8 plates. Very few objects fall outside 0 .'5, and all the bad determinations came from a PNe pair with at least one lying in a plate corner. The average error is about $0 . \prime 3$ and the corresponding mean standard deviation is about 0 .' 17 . But these objects are taken from the borders of the plates, and therefore the expected errors in the inner part of the plates should be much smaller.

Fig. 5. Histogram of the position differences for all the $\mathrm{PNe}$ present on at least 2 Schmidt plates

\subsection{Photometry}

The automatic centering program (Alard 1995) also furnishes magnitudes, by profile reconstruction of point-like sources and integration inside the profile. This method has the advantage to give good results even when the central part of the star is saturated. We have therefore directly the magnitude (in $B$ band) for all the PNe where crowding was not too important.

Calibration was obtained from $B$ and $V$ photometry of faint stars in the LMC (Linde et al. 1988) for very faint sources and from the Atlas of star clusters with color magnitude diagrams in the Magellanic Clouds (Alcaino 1975) from which we extracted 12 photometric sequences in LMC.

In total we used about 250 stars, covering a large magnitude domain from $M_{B}=9.1$ to $M_{B}=22.7$. $B$ and $V$ photometry has been used to produce $B_{\mathrm{J}}$ magnitudes for the standard stars, following the recipe given Blair \& Gilmore (1982): $B_{\mathrm{J}}=B-0.28(B-V)$.

Figure 6 shows the calibration curve with all the standard stars (except a few rejected outlying points due to confusion, saturation, etc...) and a fitted 6-degree polynomial, taken from two different plates (SERC 056J and SERC
085J). No systematic effect can be seen between these two plates. The linear domain extends from $M_{B}=18$ to 22 with a precision of about 0.20 magnitudes which is the expected internal error from the published measurements. The stars brighter than $M_{B}=16-17$ are saturated, due to the limited dynamical range of MAMA. This phenomenon is clearly seen in Fig. 6, where the slope at brighter magnitudes is smaller, and therefore the precision is diminished. But a magnitude determination is still possible down to 10-11th magnitude. From 114 stars which have been measured more than once, we derive an internal error smaller than 0.25 magnitude. Only for a few objects is the uncertainty larger, possibly due to peculiar local problems.

Fig. 6. Schmidt plates calibration

\section{The catalogue}

\subsection{LMC catalogue}

We summarize in Table 4 all our results for the 277 LMC PNe. The 4 candidates (Sa108, Sa119, Sa125 and Sa127) have been excluded (see Sect. 3.1). Excluded also are WS03 (not confirmed as a PN candidate, see SMP and Fehrenbach et al. 1978), and N016, N047 (extended), N099 (point like) VLE objects (Monk et al. 1988), but coordinates are available. The PNe are listed, first ordered by catalog names and then, within each catalogue, by increasing Right Ascension.

Column 1 indicates the selected name, with one crossidentification (first SMP, if available, then, in decreasing order of priority, Sanduleak, Jacoby, Henize, Westerlund, Lindsay, etc...). The complementary cross-identifications can then be found, if necessary, in the original papers. In Col. 2 Right Ascension and declination coordinates are given in equinox J2000 and B1950.

Column 3 is a number which gives a rough idea of the 
position error ( 1 for precision better than $0^{\prime \prime} .2,2$ between $00^{\prime \prime} 2$ and $0^{\prime \prime} 3,3$ between $0^{\prime \prime} \cdot 3$ and $0{ }^{\prime \prime} 5$, and finally 4 for coordinates less precise than 0.5 ). In parentheses, respectively, the number of position determinations (a maximum of 4), and the number of positions kept for the final averaged coordinate. The derived $B$ magnitude are listed in Col. 4 (the number of plates used is given in parentheses). Because of intense crowding, some measurements were impossible.

\section{2. $L M C$ finding charts}

The second product of this work is the complete set of finding charts for the LMC PNe. As already mentioned, only a fraction of the known PNe had published finding charts, and these were not always of usable quality. We therefore produced a homogeneous set of charts for all the objects. Plates 1-25 give the 280 finding charts taken from the IIIa-J plates of the ESO/SERC Southern Sky Atlas. Only a few exceptions (SMP098, Sa104, Mo01, Mo02, Mo49 and Mo54) are taken from Red plates. Field sizes are all $2^{\prime} \times 2^{\prime}$ and North is up and East to the left.

Note that, as a by-product, accurate positions are now also available for the standard stars.

\subsection{Cross-identification with IR sources}

As an example of possibilities opened by the knowledge of precise coordinates for $\mathrm{PNe}$, we have looked for associations with IRAS sources, as given in the catalogue of Schwering \& Israel (1990). While this is of obvious interest for the study of the PNe, it is however not illustrating the best possibilities of our catalogue, as the IRAS positions themselves are not known with an extreme accuracy. The uncertainty of the IRAS positions is given in Table 3 as the size of the error-ellipse from the Point Source Catalogue (PSC), when an association exists, as explained in the Catalogue of Schwering \& Israel. The main criterion for an association of a PN with an IRAS source is the coincidence in position. We have, somewhat arbitrarily (but nevertheless based on the size of the average error-ellipse) separated sources in 3 categories: association closer than $30^{\prime \prime}$ in distance, between $30^{\prime \prime}$ and $60^{\prime \prime}$, and between $1^{\prime}$ and $2^{\prime}$. With the density of sources in the LMC, at these larger distances however, many unrelated sources would enter the catalogue. A second criterion has thus been applied, based on the expected IR flux distribution for PNe. Following experience from galactic $\mathrm{PNe}$ (Pottasch et al. 1988), a very broad selection on flux densities can be used to select PNe: $F_{\nu} 25 \mu / F_{\nu} 12 \mu>2$ (to eliminate stars) and $F_{\nu} 25 \mu / F_{\nu} 60 \mu>0.2$ to eliminate galaxies. We have applied this criterion for those objects were the distance of the IRAS source to the optical PN was larger than $1^{\prime}$, to eliminate many spurious associa- tions. For the closer associations, we have left all of them in Table 3 , but will apply the criterion subsequently to assess the reality of the identification. When the $60 \mu$ flux was not well estimated due to confusion, the source was kept, pending further identification (a $\mathrm{C}$ is then printed instead of a flux value).

The results are given in Table 3, where we list the serial number from Schwering's list, in Col. 1, the PN name (Col. 2), the IRAS PSC cross-identification when appropriate (Col. 3) and the associated error ellipse (Cols. 4-6). If no PSC identification could be done the typical errors are $1^{\prime}$ in both RA. and Dec. (Schwering \& Israel 1990). Columns 7 to 9 give respectively the optical and IR positions (in J2000 equinox) and the distance between the two determinations (in arcsec).

Columns 10 to 13 show the IR fluxes at 12, 25, 60 and $100 \mu \mathrm{m}$, while in column 14, a "p" indicates a point-source. More details about the IRAS data can be found in the original reference (Schwering \& Israel 1990).

Zilstra et al. (1994) published a list of 12 IRAS sources associated with LMC PNe. Five of them do not show up here: SMP006, SMP061, SMP098, MG45 and Mo18, which indicates a distance between IRAS and optical position larger than $2^{\prime}$. As we used only the catalogue of Schwering \& Israël (1990), based on a special observation in the Deep Sky Mapping mode, (while Zilstra et al. used indifferently this list or the Faint Source Catalogue (FSC)), this difference has probably to be ascribed to a discrepancy between the two catalogues, which needs further investigation.

Applying now the flux ratio criteria in addition to the association with separations smaller than $1^{\prime}$, we end with a "quality criterion" (Q) for the association, given in the last column of Table 3. Quality 1 is a secure association, both because of small distances and adequate flux ratios, while quality 2 is less secure (flux ratios at the limit, or unclear due to confusion, and larger separation between optical and IR sources). Quality 3 is a doubtful association, while 0 means rejection. In the latter category enter SMP013, SMP043, SMP064, SMP069 and MG58 because the IR flux distribution indicates a stellar source (for SMP064, the IRAS source is clearly associated to the cluster NGC 1984), while for SMP092 and Mo19 the flux distribution would indicate a cold galaxy. If the two latter objects are indeed PNe, which is confirmed for SMP092 (Meatheringham \& Dopita 1991b), then they have in fact an optically thick dust shell, with a cooler exciting source, which is known to mimic the flux distribution of a galaxy. Further spectroscopy will be necessary. The same remark applies to some of the quality 3 associations in the first part of Table 3, which could be the same type objects, or lower excitation HII regions (SMP024, SMP029, SMP036, SMP075). The known (high) excitation class for the first three PNe suggests an IR excess due to a dust shell. Note that some objects are already classified as Very Low 
Table 3. Possible IRAS associations

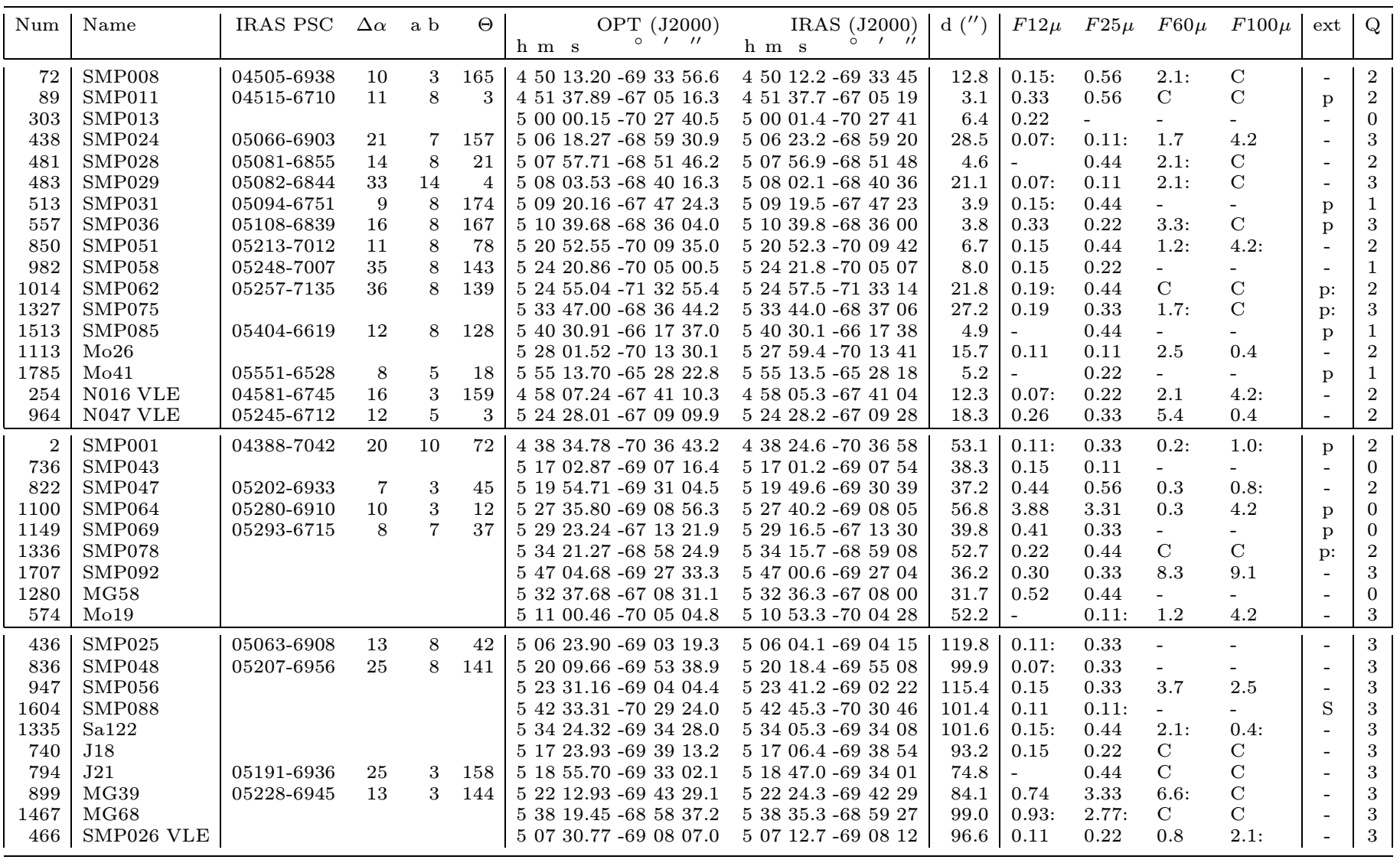

Excitation objects. It should be noted also that, because of the metallicity which is different in the Clouds compared to our Galaxy, the color criteria for the PNe might also be slightly modified. We end with 15 associations of quality 2 or better, closer than $1^{\prime}$ (among them 7 in the list of Zijlstra et al. 1994). These objects are worth further study. In the case of associations with larger separation, a close inspection of the finding charts reveals in almost all cases a better identification for the IRAS source than the PN.

\section{Conclusions}

With the last published catalogues (Morgan \& Good 1992; and Morgan 1994) the sample of known PNe in the LMC has been doubled, allowing a better study of the population of these objects in the LMC. We have provided accurate coordinates and finding charts in a homogeneous way for the whole sample. From multiple position measurements of objects lying in the overlapping parts of the Schmidt plates, we derive an accuracy better than 0."5 (with a few exceptions) and probably better than $0{ }^{\prime \prime} 3$ for most of the objects lying further away from the plate borders. This is adequate for most of the follow-up work from ground or space. It should be added that the material available will easily allow a similar, accurate measurement for any new object which should be discovered later on. An application to the identification of IRAS sources in the LMC (which have themselves poor coordinates) leads to 15 identifications with reasonable confidence, which will be further observed.

Acknowledgements. We thank Dr. D.H. Morgan for a careful reading of the manuscript. We are grateful to Rene Chesnel for scanning the Schmidt plates and support during the astrometric reductions and tests made for this program.

\section{A. Positions}

See Table 4 with LMC positions on the six next pages. 


\begin{tabular}{|c|c|c|c|c|c|c|}
\hline \multirow[t]{2}{*}{ Name } & \multicolumn{2}{|c|}{ coord OPT } & \multirow{2}{*}{\multicolumn{2}{|c|}{$\mathrm{F} \quad(\mathrm{n} 1, \mathrm{n} 2)$}} & \multirow[t]{2}{*}{$m_{B}$} & \multirow[t]{2}{*}{ (n) } \\
\hline & $\mathrm{J} 2000$ & B1950 & & & & \\
\hline SMP001=N182 & $43834.78-703643.3$ & $43859.58-704231.1$ & 1 & $(2,2)$ & 16.5 & $(1)$ \\
\hline SMP002 & $44056.67-674801.6$ & $44057.37-675340.6$ & 1 & $(3,1)$ & 17.6 & $(2)$ \\
\hline $\mathrm{SMP} 003=\mathrm{N} 001$ & $44224.09-661301.8$ & $44213.64-661835.2$ & 1 & $(1,1)$ & 16.7 & $(1)$ \\
\hline SMP004 & $44321.93-713008.2$ & 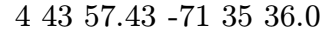 & 1 & $(2,2)$ & 18.6 & (1) \\
\hline $\mathrm{SMP} 005=\mathrm{S} 1$ & 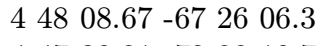 & $44808.21-673115.6$ & 1 & $(2,2)$ & 17.0 & $(2)$ \\
\hline $\mathrm{SMP} 006=\mathrm{N} 184$ & $\begin{array}{llllll}4 & 47 & 39.21 & -72 & 28 & 19.7\end{array}$ & $\begin{array}{lllll}4 & 48 & 27.21 & -72 & 33 \\
29.3\end{array}$ & 1 & $(2,2)$ & 15.8 & $(2)$ \\
\hline $\mathrm{SMP} 007=\mathrm{N} 077 \mathrm{~F}$ & $\begin{array}{llllll}4 & 48 & 29.68 & -69 & 08 & 12.6\end{array}$ & $\begin{array}{llllll}4 & 48 & 43.18 & -69 & 13 & 19.9\end{array}$ & 1 & $(1,1)$ & 16.2 & $(1)$ \\
\hline $\mathrm{SMP} 008=\mathrm{N} 078$ & $45013.20-693356.6$ & $45030.96-693856.6$ & 1 & $(1,1)$ & 16.3 & $(1)$ \\
\hline SMP009 & $45024.66-681316.0$ & $45030.84-681815.6$ & 1 & $(1,1)$ & 16.9 & (1) \\
\hline SMP010 & $\begin{array}{llllll}4 & 51 & 08.95 & -68 & 49 & 05.4\end{array}$ & 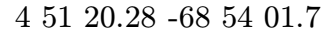 & 1 & $(1,1)$ & 17.0 & $(1)$ \\
\hline SMP011 & $45137.89-670516.3$ & $45135.54-671011.1$ & 2 & $(2,1)$ & 17.5 & $(2)$ \\
\hline SMP012 & $\begin{array}{llllll}4 & 52 & 01.48 & -68 & 39 & 16.9\end{array}$ & $45211.60-684409.6$ & 1 & $(1,1)$ & 18.1 & $(1)$ \\
\hline SMP013=WS04 & $\begin{array}{llllll}5 & 00 & 00.15 & -70 & 27 & 40.5\end{array}$ & 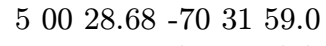 & 1 & $(1,1)$ & 16.3 & (1) \\
\hline SMP014=LM2-04 & $\begin{array}{llllll}5 & 00 & 20.82 & -70 & 58 & 50.9\end{array}$ & $\begin{array}{llllll}5 & 00 & 54.83 & -71 & 03 & 07.7\end{array}$ & 1 & $(1,1)$ & 18.7 & (1) \\
\hline $\mathrm{SMP} 015=\mathrm{N} 186 \mathrm{~A}$ & $\begin{array}{llllll}5 & 00 & 52.73 & -70 & 13 & 39.8\end{array}$ & $\begin{array}{llllll}5 & 01 & 19.10 & -70 & 17 & 54.7\end{array}$ & 1 & $(1,1)$ & 15.6 & (1) \\
\hline SMP016=LM1-11 & $\begin{array}{llllll}5 & 02 & 01.98 & -69 & 48 & 53.6\end{array}$ & $\begin{array}{lllll}5 & 02 & 24.57 & -69 & 5303.7\end{array}$ & 1 & $(1,1)$ & 17.3 & (1) \\
\hline $\mathrm{SMP} 017=\mathrm{N} 096$ & $\begin{array}{llllll}5 & 02 & 52.36 & -69 & 20 & 53.3\end{array}$ & $\begin{array}{lllll}5 & 03 & 10.75 & -69 & 24 \\
60.0\end{array}$ & 1 & $(1,1)$ & 17.6 & $(1)$ \\
\hline SMP018=L95 & $\begin{array}{llllll}5 & 03 & 42.65 & -70 & 06 & 46.8\end{array}$ & $\begin{array}{llllll}5 & 04 & 08.44 & -70 & 10 & 49.7\end{array}$ & 1 & $(1,1)$ & 14.8 & (1) \\
\hline $\mathrm{SMP} 019=\mathrm{N} 188$ & $\begin{array}{llllll}5 & 03 & 41.25 & -70 & 13 & 53.0\end{array}$ & $\begin{array}{llllll}5 & 04 & 08.21 & -70 & 17 & 55.9\end{array}$ & 1 & $(1,1)$ & 15.9 & (1) \\
\hline SMP020 & $\begin{array}{llllll}5 & 04 & 40.10 & -69 & 2139.6\end{array}$ & $\begin{array}{l}50458.94-692538.6 \\
\end{array}$ & 1 & $(1,1)$ & 18.2 & $(1)$ \\
\hline $\mathrm{SMP} 021=\mathrm{N} 097$ & $\begin{array}{llllll}5 & 04 & 52.14 & -68 & 39 & 08.6\end{array}$ & $\begin{array}{llllll}5 & 05 & 04.73 & -68 & 43 & 07.1\end{array}$ & 1 & $(1,1)$ & 16.6 & (1) \\
\hline SMP022 & $\begin{array}{llllll}5 & 05 & 50.72 & -69 & 02 & 31.1\end{array}$ & $\begin{array}{llllll}5 & 06 & 06.89 & -69 & 06 & 25.3\end{array}$ & 1 & $(1,1)$ & 18.4 & $(1)$ \\
\hline $\mathrm{SMP} 023=\mathrm{N} 024$ & $\begin{array}{llllll}5 & 06 & 09.41 & -67 & 45 & 25.7\end{array}$ & $\begin{array}{llllll}5 & 06 & 14.82 & -67 & 49 & 18.9\end{array}$ & 1 & $(2,2)$ & 16.3 & $(2)$ \\
\hline SMP024 & $\begin{array}{llllll}5 & 06 & 18.27 & -68 & 59 & 30.9\end{array}$ & $\begin{array}{llllll}5 & 06 & 34.07 & -69 & 03 & 23.1\end{array}$ & 1 & $(1,1)$ & 18.8 & (1) \\
\hline $\mathrm{SMP025}=\mathrm{L} 124$ & $\begin{array}{llllll}5 & 06 & 23.90 & -69 & 03 & 19.3\end{array}$ & $\begin{array}{llllll}5 & 06 & 40.28 & -69 & 07 & 11.1\end{array}$ & 1 & $(1,1)$ & 18.3 & $(1)$ \\
\hline SMP027 & $\begin{array}{lllll}5 & 07 & 54.94 & -6657 & 45.3\end{array}$ & $\begin{array}{lllll}507 & 54.51 & -67 & 01 & 31.3\end{array}$ & 2 & $(2,1)$ & 16.7 & $(2)$ \\
\hline SMP028 & $\begin{array}{llllll}5 & 07 & 57.71 & -68 & 51 & 46.2\end{array}$ & $\begin{array}{llllll}5 & 08 & 12.65 & -68 & 55 & 31.4\end{array}$ & 1 & $(1,1)$ & 19.0 & $(1)$ \\
\hline $\mathrm{SMP} 029=\mathrm{N} 102$ & $\begin{array}{llllll}5 & 08 & 03.53 & -68 & 40 & 16.3\end{array}$ & $\begin{array}{llllll}5 & 08 & 16.81 & -68 & 44 & 01.1\end{array}$ & 1 & $(1,1)$ & 18.4 & (1) \\
\hline SMP030=LM2-08 & $\begin{array}{llllll}5 & 09 & 10.58 & -66 & 53 & 38.2\end{array}$ & $\begin{array}{llllll}5 & 09 & 09.83 & -66 & 57 & 18.7\end{array}$ & 2 & $(2,1)$ & 17.9 & $(2)$ \\
\hline $\mathrm{SMP} 031=\mathrm{N} 025$ & $\begin{array}{llllll}5 & 09 & 20.16 & -67 & 47 & 24.3\end{array}$ & 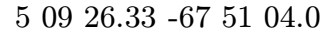 & 2 & $(2,1)$ & 14.7 & $(2)$ \\
\hline $\mathrm{SMP} 032=\mathrm{N} 192$ & $\begin{array}{llllll}5 & 09 & 37.23 & -70 & 49 & 08.1\end{array}$ & 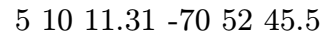 & 1 & $(1,1)$ & 16.9 & (1) \\
\hline $\mathrm{SMP} 033=\mathrm{N} 104 \mathrm{~A}$ & $\begin{array}{llllll}5 & 10 & 09.48 & -68 & 29 & 54.0\end{array}$ & $\begin{array}{llllll}5 & 10 & 21.62 & -68 & 33 & 29.9\end{array}$ & 1 & $(1,1)$ & 16.5 & $(1)$ \\
\hline $\mathrm{SMP} 034=\mathrm{N} 106$ & $\begin{array}{llllll}5 & 10 & 17.16 & -68 & 48 & 22.5\end{array}$ & $\begin{array}{llllll}5 & 10 & 31.98 & -68 & 51 & 57.8\end{array}$ & 1 & $(1,1)$ & 18.7 & (1) \\
\hline $\mathrm{SMP} 035=\mathrm{WS} 12$ & $\begin{array}{llllll}5 & 10 & 49.97 & -65 & 29 & 30.4\end{array}$ & $\begin{array}{lllll}5 & 10 & 39.61 & -6533 & 04.3\end{array}$ & 1 & $(1,1)$ & 15.4 & (1) \\
\hline $\mathrm{SMP} 036=\mathrm{N} 107$ & $\begin{array}{llllll}5 & 10 & 39.68 & -68 & 36 & 04.0\end{array}$ & $\begin{array}{llllll}5 & 10 & 52.78 & -68 & 39 & 37.7\end{array}$ & 1 & $(1,1)$ & 16.1 & (1) \\
\hline $\mathrm{SMP} 037=\mathrm{N} 028$ & $\begin{array}{llllll}5 & 11 & 02.89 & -67 & 47 & 58.8\end{array}$ & $\begin{array}{lllll}5 & 11 & 09.39 & -67 & 51.2\end{array}$ & 1 & $(2,2)$ & 15.2 & $(2)$ \\
\hline $\mathrm{SMP} 038=\mathrm{N} 110$ & $\begin{array}{lllll}5 & 1123.78 & -700156.5\end{array}$ & $\begin{array}{lllll}5 & 1150.14 & -70 & 0526.6\end{array}$ & 1 & $(1,1)$ & 16.0 & (1) \\
\hline SMP039=?LM2-11 & $\begin{array}{llllll}5 & 11 & 42.14 & -68 & 34 & 59.7\end{array}$ & $\begin{array}{llllll}5 & 11 & 55.24 & -68 & 38 & 29.0\end{array}$ & 1 & $(1,1)$ & 17.8 & (1) \\
\hline $\mathrm{SMP} 040=\mathrm{WS} 16$ & $\begin{array}{llllll}5 & 12 & 15.76 & -66 & 22 & 56.1\end{array}$ & 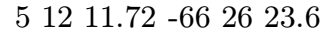 & 1 & $(1,1)$ & 17.0 & $(1)$ \\
\hline SMP041=LM1-26 & $\begin{array}{llllll}5 & 13 & 27.33 & -70 & 33 & 34.7\end{array}$ & $\begin{array}{lllll}5 & 13 & 59.34 & -70 & 365.8\end{array}$ & 1 & $(1,1)$ & 14.7 & (1) \\
\hline SMP042 & $\begin{array}{llllll}5 & 15 & 46.81 & -68 & 42 & 23.7\end{array}$ & 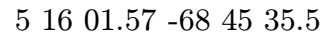 & 1 & $(1,1)$ & 16.8 & (1) \\
\hline SMP043 & $\begin{array}{llllll}5 & 17 & 02.87 & -69 & 07 & 16.4\end{array}$ & $\begin{array}{llllll}5 & 17 & 21.49 & -69 & 1022.6\end{array}$ & 1 & $(1,1)$ & 19.9 & (1) \\
\hline SMP044=LM2-16 & $\begin{array}{llllll}5 & 18 & 29.94 & -67 & 16 & 55.8\end{array}$ & 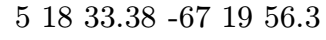 & 1 & $(2,2)$ & 17.0 & $(2)$ \\
\hline $\mathrm{SMP} 045=\mathrm{WS} 17$ & $\begin{array}{llllll}5 & 19 & 20.73 & -66 & 58 & 07.5\end{array}$ & $\begin{array}{llllll}5 & 19 & 21.87 & -67 & 01 & 04.4\end{array}$ & 2 & $(2,1)$ & 16.1 & $(2)$ \\
\hline SMP046 & $\begin{array}{llllll}5 & 19 & 29.70 & -68 & 51 & 07.9\end{array}$ & 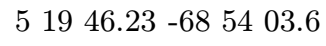 & 1 & $(1,1)$ & 18.9 & (1) \\
\hline $\mathrm{SMP} 047=\mathrm{N} 122$ & $\begin{array}{llllll}5 & 19 & 54.71 & -69 & 31 & 04.5\end{array}$ & 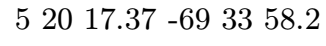 & 1: & $(1,1)$ & 00.0 & (0) \\
\hline $\mathrm{SMP} 048=\mathrm{N} 123$ & $\begin{array}{llllll}5 & 20 & 09.66 & -69 & 53 & 38.9\end{array}$ & $\begin{array}{llllll}5 & 20 & 35.96 & -69 & 56 & 31.4\end{array}$ & 1: & $(1,1)$ & 00.0 & $(0)$ \\
\hline $\mathrm{SMP049=N196}$ & $\begin{array}{llllll}5 & 20 & 09.36 & -70 & 25 & 38.1\end{array}$ & $\begin{array}{llllll}5 & 20 & 40.99 & -70 & 28 & 30.4\end{array}$ & 1 & $(1,1)$ & 17.5 & (1) \\
\hline $\mathrm{SMP} 050=\mathrm{N} 039$ & $\begin{array}{llllll}5 & 20 & 51.73 & -67 & 05 & 42.5\end{array}$ & $\begin{array}{llllll}5 & 20 & 54.00 & -67 & 08 & 32.9\end{array}$ & 2 & $(3,2)$ & 15.3 & (3) \\
\hline $\mathrm{SMP} 051=\mathrm{N} 125$ & $\begin{array}{llllll}5 & 20 & 52.55 & -70 & 09 & 35.0\end{array}$ & 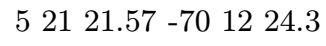 & 1 & $(1,1)$ & 17.7 & (1) \\
\hline $\mathrm{SMP} 052=\mathrm{N} 124$ & $\begin{array}{llllll}5 & 21 & 23.83 & -68 & 35 & 34.4 \\
\end{array}$ & 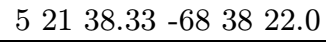 & 1 & $(1,1)$ & 15.1 & $(1)$ \\
\hline
\end{tabular}




\begin{tabular}{|c|c|c|c|c|c|c|}
\hline \multicolumn{7}{|c|}{ continued from previous page } \\
\hline \multirow[t]{2}{*}{ Name } & \multicolumn{2}{|c|}{ coord OPT } & \multirow{2}{*}{\multicolumn{2}{|c|}{$\mathrm{F} \quad(\mathrm{n} 1, \mathrm{n} 2)$}} & \multirow[t]{2}{*}{$m_{B}$} & \multirow[t]{2}{*}{$(\mathrm{n})$} \\
\hline & J2000 & B1950 & & & & \\
\hline SMP053=N042 & $\begin{array}{llllll}5 & 21 & 32.89 & -67 & 00 & 04.0\end{array}$ & $52134.53-670251.4$ & 2 & $(3,1)$ & 16.3 & $(3)$ \\
\hline $\mathrm{SMP} 054=\mathrm{J} 35$ & $\begin{array}{lllll}521 & 42.93 & -68 & 39 & 24.8\end{array}$ & $\begin{array}{lllll}521 & 58.02 & -68 & 42 & 11.0\end{array}$ & 1 & $(1,1)$ & 17.0 & (1) \\
\hline $\mathrm{SMP} 055=\mathrm{N} 199$ & $\begin{array}{llllll}522 & 40.96 & -71 & 19 & 06.7\end{array}$ & $\begin{array}{llllll}52322.52 & -71 & 21 & 47.7\end{array}$ & 1 & $(1,1)$ & 17.1 & $(1)$ \\
\hline $\mathrm{SMP} 056=\mathrm{N} 136$ & $\begin{array}{lllllll}5 & 23 & 31.16 & -69 & 04 & 04.4\end{array}$ & 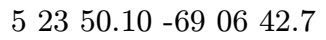 & 1 & $(1,1)$ & 18.1 & (1) \\
\hline SMP057 & $\begin{array}{lllll}523 & 48.62 & -69 & 12 & 21.6\end{array}$ & $52408.86-691458.5$ & 1 & $(1,1)$ & 18.0 & (1) \\
\hline $\mathrm{SMP} 058=\mathrm{N} 013$ & $52420.86-700500.5$ & $\begin{array}{lllll}524 & 49.55 & -70 & 07 & 34.8\end{array}$ & 1 & $(1,1)$ & 19.4 & $(1)$ \\
\hline SMP059=LM2-25 & $\begin{array}{lllll}524 & 27.35 & -70 & 22 & 23.7\end{array}$ & $52458.98-702457.4$ & 1 & $(1,1)$ & 17.3 & (1) \\
\hline SMP060 & $\begin{array}{lllll}524 & 15.69 & -70 & 5356.3\end{array}$ & $52452.83-705630.8$ & 1 & $(1,1)$ & 16.5 & (1) \\
\hline SMP061=N203 & 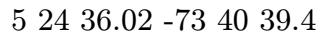 & $\begin{array}{llllll}5 & 25 & 48.13 & -73 & 43 & 11.1\end{array}$ & 1 & $(1,1)$ & 16.2 & (1) \\
\hline $\mathrm{SMP} 062=\mathrm{N} 201$ & 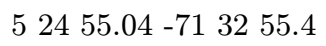 & 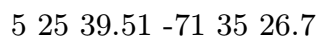 & 2 & $(2,1)$ & 15.0 & $(2)$ \\
\hline $\mathrm{SMP} 063=\mathrm{N} 141$ & $\begin{array}{lllll}525 & 26.18 & -68 & 55 & 53.8\end{array}$ & $\begin{array}{llllll}5 & 25 & 44.12 & -68 & 58 & 23.8\end{array}$ & 1 & $(1,1)$ & 16.6 & (1) \\
\hline $\mathrm{SMP} 064=\mathrm{N} 145$ & $\begin{array}{llllll}52 & 275.80 & -69 & 08 & 56.3\end{array}$ & $\begin{array}{llllll}5 & 27 & 55.93 & -69 & 11 & 16.9\end{array}$ & 1 & $(1,1)$ & 18.7 & (1) \\
\hline SMP065 & $\begin{array}{llllll}5 & 27 & 43.83 & -71 & 25 & 56.0\end{array}$ & $\begin{array}{llllll}5 & 28 & 27.29 & -71 & 28 & 15.2\end{array}$ & 2 & $(2,1)$ & 17.7 & $(2)$ \\
\hline $\mathrm{SMP} 066=\mathrm{N} 052$ & $\begin{array}{llllll}5 & 28 & 41.15 & -67 & 33 & 39.1\end{array}$ & $\begin{array}{llllll}5 & 28 & 47.85 & -67 & 35 & 55.4\end{array}$ & 2 & $(3,2)$ & 16.2 & $(3)$ \\
\hline $\mathrm{SMP} 067=\mathrm{N} 053$ & $\begin{array}{llllll}5 & 29 & 15.67 & -67 & 32 & 46.7\end{array}$ & $\begin{array}{lllll}529 & 22.31 & -67 & 3500.6\end{array}$ & 2 & $(3,2)$ & 16.9 & $(3)$ \\
\hline SMP068 & $\begin{array}{llllll}5 & 29 & 02.85 & -70 & 19 & 24.8\end{array}$ & $\begin{array}{llllll}5 & 29 & 34.48 & -70 & 21 & 38.7\end{array}$ & 1 & $(2,2)$ & 19.3 & $(2)$ \\
\hline $\mathrm{SMP} 069=\mathrm{N} 054$ & 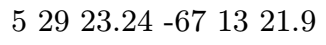 & $\begin{array}{llllll}5 & 29 & 27.35 & -67 & 15 & 35.3\end{array}$ & 2 & $(3,1)$ & 15.4 & (3) \\
\hline SMP070=LM2-28 & $\begin{array}{lllll}529 & 26.64 & -72 & 38 & 42.6\end{array}$ & 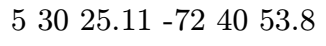 & 1 & $(3,3)$ & 18.4 & (3) \\
\hline SMP071=N207 & $\begin{array}{lllll}5 & 30 & 33.30 & -70 & 44 \\
37.6\end{array}$ & $\begin{array}{llllll}5 & 31 & 09.47 & -70 & 46 & 44.8\end{array}$ & 2 & $(2,1)$ & 16.0 & $(2)$ \\
\hline SMP072 & $\begin{array}{lllll}530 & 45.83 & -70 & 50 & 15.8\end{array}$ & 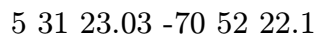 & 1 & $(2,2)$ & 16.4 & $(2)$ \\
\hline $\mathrm{SMP} 073=\mathrm{N} 208$ & $\begin{array}{llllll}5 & 31 & 21.97 & -70 & 40 & 44.9\end{array}$ & $53157.53-704248.6$ & 2 & $(2,1)$ & 14.4 & $(2)$ \\
\hline $\mathrm{SMP} 074=\mathrm{N} 209$ & $\begin{array}{lllll}53329.84 & -715228.4\end{array}$ & $53419.09-715422.3$ & 1 & $(2,2)$ & 14.9 & $(2)$ \\
\hline $\mathrm{SMP} 075=\mathrm{N} 151$ & $\begin{array}{llllll}5 & 33 & 47.00 & -68 & 36 & 44.2\end{array}$ & $\begin{array}{llllll}5 & 34 & 02.90 & -68 & 38 & 38.1\end{array}$ & 2 & $(2,1)$ & 15.8 & $(2)$ \\
\hline $\mathrm{SMP} 076=\mathrm{N} 060$ & $\begin{array}{llllll}5 & 33 & 56.17 & -67 & 53 & 08.3\end{array}$ & 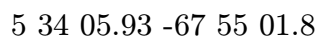 & 2 & $(4,2)$ & 16.6 & (4) \\
\hline $\mathrm{SMP} 077=\mathrm{N} 152$ & $\begin{array}{llllll}5 & 34 & 06.28 & -69 & 26 & 17.7\end{array}$ & $\begin{array}{lllll}534 & 29.69 & -69 & 28 & 09.9\end{array}$ & 1 & $(2,2)$ & 17.7 & $(2)$ \\
\hline $\mathrm{SMP} 078=\mathrm{N} 153$ & 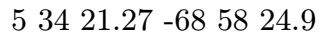 & $\begin{array}{llllll}5 & 34 & 40.41 & -69 & 00 & 16.2\end{array}$ & 2 & $(2,1)$ & 16.8 & $(2)$ \\
\hline $\mathrm{SMP} 079=\mathrm{N} 210$ & $53408.73-742006.5$ & $\begin{array}{lllll}5 & 3532.07 & -74 & 2156.4\end{array}$ & 1 & $(1,1)$ & 16.3 & (1) \\
\hline SMP080 & $53438.95-701955.5$ & $\begin{array}{llllll}5 & 35 & 11.18 & -70 & 21 & 45.0\end{array}$ & 1 & $(2,2)$ & 18.5 & $(2)$ \\
\hline $\mathrm{SMP} 081=\mathrm{N} 211$ & $53520.95-735529.6$ & $53638.04-735714.5$ & 1 & $(1,1)$ & 16.3 & (1) \\
\hline SMP082=LM2-38 & 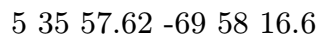 & $\begin{array}{lllll}536 & 26.32 & -70 & 00 & 00.6\end{array}$ & 1 & $(2,2)$ & 19.3 & $(2)$ \\
\hline $\mathrm{SMP} 083=\mathrm{N} 066$ & $\begin{array}{llllll}5 & 36 & 20.81 & -67 & 18 & 07.5\end{array}$ & $\begin{array}{lllll}5 & 36 & 26.08 & -67 & 19 \\
50.6\end{array}$ & 1 & $(4,4)$ & 15.5 & (4) \\
\hline $\mathrm{SMP} 084=\mathrm{N} 212$ & $53653.02-715338.0$ & $\begin{array}{lllll}537 & 42.80 & -71 & 5517.2\end{array}$ & 2 & $(3,2)$ & 16.3 & (3) \\
\hline $\mathrm{SMP} 085=\mathrm{N} 069$ & $\begin{array}{llllll}5 & 40 & 30.91 & -66 & 17 & 37.0\end{array}$ & $\begin{array}{llllll}5 & 40 & 28.87 & -66 & 19 & 02.2\end{array}$ & 1 & $(1,1)$ & 17.4 & (1) \\
\hline SMP086=LM2-41 & $\begin{array}{llllll}5 & 41 & 22.05 & -68 & 07 & 44.2\end{array}$ & $\begin{array}{llllll}5 & 41 & 34.31 & -68 & 09 & 05.3\end{array}$ & 2 & $(3,2)$ & 18.7 & $(3)$ \\
\hline $\mathrm{SMP} 087=\mathrm{N} 215$ & 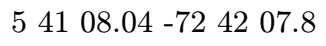 & $\begin{array}{llllll}5 & 42 & 08.32 & -72 & 43 & 28.1\end{array}$ & 1 & $(3,3)$ & 17.1 & (3) \\
\hline SMP088=LM2-42 & $\begin{array}{llllll}5 & 42 & 33.31 & -70 & 29 & 24.0\end{array}$ & $\begin{array}{llllll}5 & 43 & 07.73 & -70 & 30 & 39.0\end{array}$ & 1 & $(2,2)$ & 16.2 & $(2)$ \\
\hline $\mathrm{SMP} 089=\mathrm{N} 178$ & 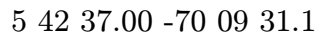 & $\begin{array}{llllll}5 & 43 & 08.02 & -70 & 10 & 46.0\end{array}$ & 1 & $(2,2)$ & 14.8 & $(2)$ \\
\hline SMP090=LM2-43 & $\begin{array}{llllll}5 & 44 & 34.76 & -70 & 21 & 40.0\end{array}$ & $\begin{array}{llllll}5 & 45 & 07.95 & -70 & 22 & 46.3\end{array}$ & 2 & $(2,1)$ & 18.1 & $(2)$ \\
\hline SMP091=LM1-57 & $\begin{array}{llllll}5 & 45 & 05.97 & -68 & 06 & 50.9\end{array}$ & $\begin{array}{llllll}5 & 45 & 18.28 & -68 & 07 & 55.7\end{array}$ & 2 & $(3,2)$ & 17.2 & $(3)$ \\
\hline $\mathrm{SMP} 092=\mathrm{N} 170$ & $\begin{array}{llllll}5 & 47 & 04.68 & -69 & 27 & 33.3\end{array}$ & $\begin{array}{llllll}5 & 47 & 29.06 & -69 & 28 & 29.0\end{array}$ & 2 & $(2,1)$ & 15.6 & $(2)$ \\
\hline $\mathrm{SMP} 093=\mathrm{N} 181$ & $\begin{array}{llllll}5 & 49 & 38.75 & -69 & 09 & 59.3\end{array}$ & $\begin{array}{llllll}5 & 50 & 00.48 & -69 & 10 & 43.9\end{array}$ & 1 & $(1,1)$ & 17.3 & (1) \\
\hline SMP094=LM2-44 & $\begin{array}{llllll}5 & 54 & 10.73 & -73 & 02 & 47.4\end{array}$ & $\begin{array}{llllll}5 & 55 & 16.17 & -73 & 03 & 10.6\end{array}$ & 1 & $(2,2)$ & 16.1 & $(2)$ \\
\hline SMP095 & $\begin{array}{llllll}6 & 01 & 45.34 & -67 & 56 & 06.4\end{array}$ & $60156.42-675558.4$ & 1 & $(2,2)$ & 18.1 & $(2)$ \\
\hline SMP096 & $\begin{array}{llllll}6 & 06 & 05.45 & -71 & 04 & 14.0\end{array}$ & $\begin{array}{llllll}6 & 06 & 46.42 & -71 & 03 & 45.9\end{array}$ & 1 & $(1,1)$ & 18.1 & (1) \\
\hline $\mathrm{SMP} 097=\mathrm{WS} 40$ & $\begin{array}{llllll}6 & 10 & 25.47 & -67 & 56 & 20.4\end{array}$ & $61036.37-675534.5$ & 2 & $(2,1)$ & 17.3 & $(2)$ \\
\hline SMP098=WS41 & $\begin{array}{llllll}6 & 17 & 35.60 & -73 & 12 & 34.1\end{array}$ & $\begin{array}{llllll}6 & 18 & 42.46 & -73 & 11 & 14.9\end{array}$ & 1 & $(1,1)$ & 00.0 & $(0)$ \\
\hline $\mathrm{SMP} 099=\mathrm{N} 221$ & $\begin{array}{llllll}6 & 18 & 58.05 & -71 & 35 & 50.1\end{array}$ & $\begin{array}{llllll}6 & 19 & 44.28 & -713425.6\end{array}$ & 1 & $(1,1)$ & 16.3 & (1) \\
\hline SMP100=LM1-65 & $62255.50-720740.5$ & 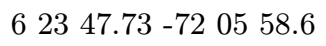 & 1 & $(1,1)$ & 17.3 & (1) \\
\hline SMP101 & $\begin{array}{llllll}6 & 23 & 40.33 & -69 & 10 & 37.8\end{array}$ & 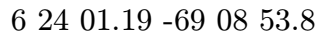 & 1 & $(1,1)$ & 17.5 & (1) \\
\hline SMP102 & 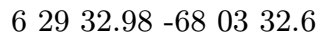 & $\begin{array}{llllll}6 & 29 & 43.57 & -68 & 01 & 23.4\end{array}$ & 1 & $(1,1)$ & 18.6 & (1) \\
\hline $\mathrm{Sa} 104=\mathrm{LM} 1-01$ & $42437.55-694220.7$ & $42449.60-694905.6$ & 1 & $(1,1)$ & 00.0 & $(0)$ \\
\hline Sa105 & $\begin{array}{llllll}5 & 02 & 32.90 & -69 & 26 & 15.2\end{array}$ & $\begin{array}{llllll}5 & 02 & 52.06 & -69 & 30 & 23.2\end{array}$ & 1 & $(1,1)$ & 19.0 & (1) \\
\hline & con & on next page & & & & \\
\hline
\end{tabular}




\begin{tabular}{|c|c|c|c|c|c|c|}
\hline \multicolumn{7}{|c|}{ continued from previous page } \\
\hline \multirow[t]{2}{*}{ Name } & \multicolumn{2}{|c|}{ coord OPT } & \multirow{2}{*}{\multicolumn{2}{|c|}{$\mathrm{F} \quad(\mathrm{n} 1, \mathrm{n} 2)$}} & \multirow{2}{*}{$m_{B}$} & \multirow[t]{2}{*}{$(\mathrm{n})$} \\
\hline & $\mathrm{J} 2000$ & B1950 & & & & \\
\hline Sa106 & $\begin{array}{llllll}5 & 03 & 05.75 & -68 & 33 & 37.0\end{array}$ & $\begin{array}{lllllll}5 & 03 & 17.24 & -68 & 37 & 43.0\end{array}$ & 1 & $(1,1)$ & 19.3 & (1) \\
\hline Sa107=LM2-06 & 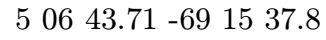 & 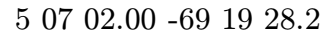 & 1 & $(1,1)$ & 18.2 & (1) \\
\hline $\mathrm{Sa} 109=\mathrm{J} 05$ & $\begin{array}{llllll}5 & 11 & 48.12 & -69 & 23 & 43.1\end{array}$ & $\begin{array}{llllll}5 & 12 & 08.48 & -69 & 27 & 11.7\end{array}$ & 1 & $(1,1)$ & 18.4 & (1) \\
\hline Sa110 & $\begin{array}{llllll}5 & 12 & 16.72 & -68 & 29 & 10.1\end{array}$ & 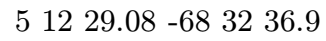 & 1 & $(1,1)$ & 18.7 & (1) \\
\hline Sa117 & $52456.74-691531.2$ & 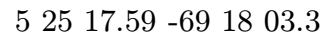 & 1 & $(1,1)$ & 19.9 & (1) \\
\hline Sa120 & $\begin{array}{llllll}5 & 29 & 32.70 & -70 & 17 & 39.0\end{array}$ & 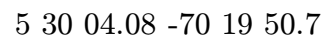 & 1 & $(2,2)$ & 20.5 & $(2)$ \\
\hline Sa121 & 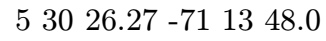 & $53107.75-71 \quad 1555.5$ & 1 & $(2,2)$ & 19.1 & $(2)$ \\
\hline Sa122 & $53424.32-693428.0$ & $53449.05-693618.9$ & 2 & $(2,1)$ & 19.1 & $(2)$ \\
\hline Sa123 & $53430.17-702834.5$ & 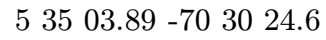 & 1 & $(2,2)$ & 18.7 & $(2)$ \\
\hline $\mathrm{Sa} 124$ & $\begin{array}{llllll}5 & 40 & 44.59 & -67 & 18 & 07.7\end{array}$ & $\begin{array}{llllll}5 & 40 & 50.12 & -67 & 19 & 31.7\end{array}$ & 2 & $(4,3)$ & 19.4 & (4) \\
\hline Sa126 & $\begin{array}{llllll}5 & 53 & 14.61 & -70 & 25 & 02.0\end{array}$ & 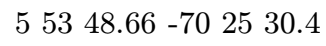 & 1 & $(1,1)$ & 19.1 & (1) \\
\hline Sa104a & $42531.9-66 \quad 47 \quad 18$ & $42521.3-665400$ & 4 & $(0,0)$ & 00.0 & $(0)$ \\
\hline J04 & 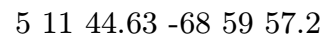 & $\begin{array}{llllll}5 & 12 & 01.38 & -69 & 03 & 26.1\end{array}$ & 1 & $(1,1)$ & 21.1 & (1) \\
\hline J07 & $\begin{array}{llllll}5 & 11 & 55.89 & -69 & 07 & 30.8\end{array}$ & $\begin{array}{llllll}5 & 12 & 13.80 & -69 & 10 & 58.9\end{array}$ & 1 & $(1,1)$ & 21.7 & (1) \\
\hline J10 & $\begin{array}{llllll}5 & 12 & 59.89 & -68 & 57 & 07.7\end{array}$ & $\begin{array}{llllll}5 & 13 & 16.41 & -69 & 00 & 31.3\end{array}$ & 1 & $(1,1)$ & 21.2 & (1) \\
\hline $\mathrm{J} 12$ & 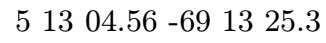 & $51323.53-691648.5$ & 1: & $(1,1)$ & 00.0 & $(0)$ \\
\hline J14 & 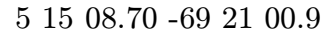 & $\begin{array}{l}51529.14-692415.2\end{array}$ & 1 & $(1,1)$ & 20.4 & (1) \\
\hline $\mathrm{J} 15=\mathrm{Sa} 111$ & $\begin{array}{llllll}5 & 15 & 24.85 & -69 & 28 & 43.1\end{array}$ & $51546.53-693156.2$ & 1 & $(1,1)$ & 20.1 & (1) \\
\hline J16 & $\begin{array}{llllll}5 & 15 & 54.68 & -69 & 30 & 35.7\end{array}$ & $\begin{array}{llllll}5 & 16 & 16.72 & -69 & 33 & 46.6\end{array}$ & 1 & $(1,1)$ & 00.0 & $(0)$ \\
\hline $\mathrm{J} 17$ & $\begin{array}{llllll}5 & 17 & 00.72 & -69 & 19 & 29.1\end{array}$ & 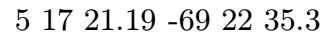 & 1 & $(1,1)$ & 20.6 & (1) \\
\hline J18 & $\begin{array}{lllll}5 & 17 & 23.93 & -69 & 39 \\
13.2\end{array}$ & $\begin{array}{llllll}5 & 17 & 47.54 & -69 & 42 & 17.7\end{array}$ & 1 & $(1,1)$ & 00.0 & $(0)$ \\
\hline J19 & $\begin{array}{llllll}5 & 17 & 58.87 & -69 & 39 & 23.0\end{array}$ & $\begin{array}{lllll}5 & 1822.59 & -69 & 42 & 25.0\end{array}$ & 1 & $(1,1)$ & 21.1 & (1) \\
\hline $\mathrm{J} 20=\mathrm{Sa} 112$ & $\begin{array}{llllll}5 & 18 & 45.78 & -69 & 10 & 11.5\end{array}$ & $\begin{array}{lllll}5 & 19 & 05.07 & -69 & 13 \\
10.3\end{array}$ & 1 & $(1,1)$ & 20.6 & (1) \\
\hline $\mathrm{J} 21$ & $\begin{array}{llllll}5 & 18 & 55.70 & -69 & 33 & 02.1\end{array}$ & 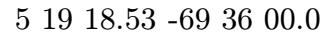 & 1 & $(1,1)$ & 00.0 & $(0)$ \\
\hline $\mathrm{J} 22$ & $\begin{array}{lllll}5 & 19 & 07.02 & -69 & 41 \\
54.0\end{array}$ & $\begin{array}{lllll}5 & 19 & 31.28 & -69 & 44 \\
51.1\end{array}$ & 1: & $(1,1)$ & 00.0 & $(0)$ \\
\hline $\mathrm{J} 23$ & $\begin{array}{lllll}5 & 19 & 15.08 & -69 & 34 \\
52.6\end{array}$ & $\begin{array}{llllll}5 & 19 & 38.25 & -69 & 37 & 49.1\end{array}$ & 1 & $(1,1)$ & 00.0 & $(0)$ \\
\hline $\mathrm{J} 24$ & $\begin{array}{lllll}5 & 19 & 34.18 & -69 & 44 \\
58.9\end{array}$ & 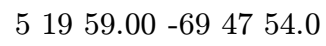 & 1 & $(1,1)$ & 00.0 & $(0)$ \\
\hline $\mathrm{J} 26=\mathrm{Sa} 113$ & 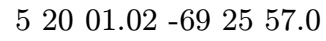 & 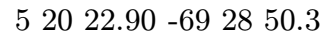 & 1: & $(1,1)$ & 00.0 & $(0)$ \\
\hline $\mathrm{J} 31=\mathrm{Sa} 114$ & 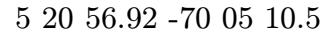 & 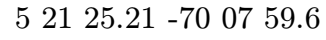 & 1 & $(1,1)$ & 19.4 & (1) \\
\hline J32 & $\begin{array}{lllll}5 & 21 & 07.64 & -69 & 44 \\
28.1\end{array}$ & $\begin{array}{lllll}5 & 2132.58 & -69 & 47 & 16.5\end{array}$ & 1 & $(1,1)$ & 00.0 & $(0)$ \\
\hline $\mathrm{J} 33=\mathrm{Sa} 115$ & $52117.55-694301.0$ & 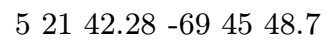 & 1 & $(1,1)$ & 00.0 & $(0)$ \\
\hline $\mathrm{J} 38=\mathrm{Sa} 116$ & 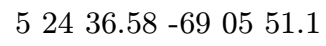 & 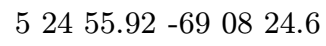 & 1 & $(1,1)$ & 19.0 & (1) \\
\hline $\mathrm{J} 41=\mathrm{Sa} 118$ & 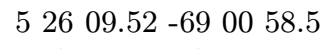 & 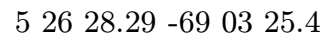 & 1 & $(1,1)$ & 18.0 & (1) \\
\hline MG01 & $44722.84-674118.8$ & $44724.15-674631.1$ & 2 & $(3,1)$ & 19.3 & $(2)$ \\
\hline MG02 & $\begin{array}{llllll}4 & 48 & 09.25 & -68 & 33 & 39.0\end{array}$ & 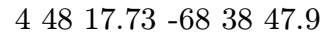 & 1 & $(2,1)$ & 19.6 & (1) \\
\hline MG03 & $45056.72-661952.5$ & $45048.82-662450.3$ & 1 & $(1,1)$ & 19.1 & (1) \\
\hline MG04 & $45245.24-701749.1$ & $45310.54-702238.2$ & 1 & $(1,1)$ & 19.9 & (1) \\
\hline MG05 & $45329.66-682252.3$ & $45337.80-682738.9$ & 1 & $(1,1)$ & 19.0 & (1) \\
\hline MG06 & 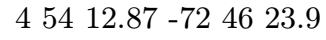 & 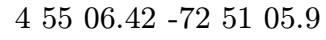 & 1 & $(2,2)$ & 18.5 & $(2)$ \\
\hline MG07 & $45409.75-745950.7$ & $\begin{array}{lllll}4 & 5535.98 & -75 & 04 & 31.7\end{array}$ & 1 & $(1,1)$ & 19.1 & (1) \\
\hline MG08 & $45539.79-683420.3$ & $45549.97-683857.8$ & 1 & $(1,1)$ & 18.4 & (1) \\
\hline MG09 & $45621.09-672422.5$ & $45622.05-672857.3$ & 1 & $(2,2)$ & 20.1 & $(2)$ \\
\hline MG10 & $45637.79-674054.6$ & $45640.93-674528.2$ & 1 & $(2,2)$ & 19.5 & (2) \\
\hline MG11 & 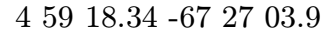 & $45920.18-673126.3$ & 1 & $(2,2)$ & 19.4 & $(2)$ \\
\hline MG12 & 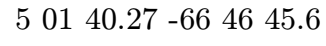 & $\begin{array}{llll}50137.50 & -66 & 50 & 58.2\end{array}$ & 1 & $(1,1)$ & 20.0 & (1) \\
\hline MG13 & 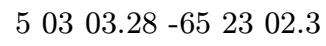 & 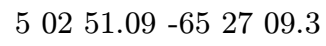 & 1 & $(1,1)$ & 19.1 & (1) \\
\hline MG14 & 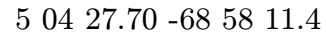 & 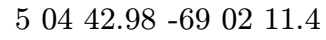 & 1 & $(1,1)$ & 19.2 & (1) \\
\hline MG15 & 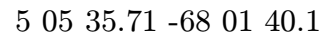 & $\begin{array}{llllll}5 & 05 & 43.21 & -68 & 05 & 35.6\end{array}$ & 1 & $(2,2)$ & 19.3 & $(2)$ \\
\hline MG16 & $\begin{array}{llllll}5 & 06 & 05.18 & -64 & 48 & 49.2\end{array}$ & $\begin{array}{llllll}5 & 05 & 49.78 & -64 & 52 & 43.4\end{array}$ & 1 & $(1,1)$ & 19.2 & (1) \\
\hline MG17 & 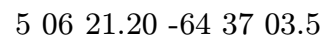 & 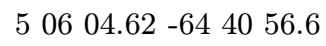 & 1 & $(1,1)$ & 19.6 & (1) \\
\hline MG18 & 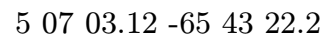 & 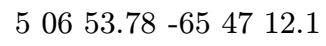 & 1 & $(1,1)$ & 19.7 & (1) \\
\hline MG19 & $\begin{array}{llllll}5 & 08 & 32.04 & -68 & 09 & 44.3\end{array}$ & 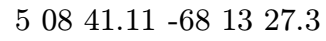 & 1 & $(1,1)$ & 19.8 & (1) \\
\hline
\end{tabular}




\begin{tabular}{|c|c|c|c|c|c|c|}
\hline \multicolumn{7}{|c|}{ continued from previous page } \\
\hline \multirow[t]{2}{*}{ Name } & \multicolumn{2}{|c|}{ coord OPT } & \multirow{2}{*}{\multicolumn{2}{|c|}{$\mathrm{F} \quad(\mathrm{n} 1, \mathrm{n} 2)$}} & \multirow{2}{*}{$m_{B}$} & \multirow[t]{2}{*}{ (n) } \\
\hline & $\mathrm{J} 2000$ & B1950 & & & & \\
\hline MG20 & $\begin{array}{llllll} & 10 & 40.24 & -68 & 10 & 23.5\end{array}$ & $\begin{array}{llllll} & 10 & 49.73 & -68 & 13 & 57.3\end{array}$ & 1 & $(1,1)$ & 19.7 & $(1)$ \\
\hline MG21 & $51137.95-654242.1$ & $\begin{array}{llllll}5 & 11 & 29.17 & -65 & 46 & 12.4\end{array}$ & 1 & $(1,1)$ & 18.5 & (1) \\
\hline MG22 & $51153.52-653227.0$ & $\begin{array}{llllll}5 & 11 & 43.63 & -65 & 35 & 56.3\end{array}$ & 1 & $(1,1)$ & 19.4 & (1) \\
\hline MG23 & $51147.39-681609.5$ & $51157.85-681938.5$ & 1 & $(1,1)$ & 18.3 & (1) \\
\hline MG24 & $\begin{array}{llllll}5 & 13 & 01.01 & -65 & 15 & 34.1\end{array}$ & $\begin{array}{llllll}5 & 12 & 49.40 & -65 & 18 & 58.7\end{array}$ & 1 & $(1,1)$ & 18.8 & (1) \\
\hline MG25 & $51217.83-715449.5$ & $\begin{array}{llllll}5 & 13 & 04.68 & -7158 & 15.0\end{array}$ & 2 & $(2,1)$ & 19.3 & $(2)$ \\
\hline MG26 & $51328.00-661728.4$ & $\begin{array}{llllll}5 & 13 & 23.47 & -66 & 20 & 50.8\end{array}$ & 1 & $(1,1)$ & 17.9 & (1) \\
\hline MG27 & $51334.23-653513.2$ & $\begin{array}{llllll}5 & 1324.85 & -6538 & 35.3\end{array}$ & 1 & $(1,1)$ & 19.7 & (1) \\
\hline MG28 & $\begin{array}{llllll}5 & 13 & 09.74 & -69 & 31 & 18.0\end{array}$ & $\begin{array}{llllll}5 & 13 & 31.48 & -69 & 34 & 40.7\end{array}$ & 1 & $(1,1)$ & 19.2 & (1) \\
\hline MG29 & $\begin{array}{llllll}5 & 13 & 42.35 & -68 & 15 & 16.4\end{array}$ & $\begin{array}{llllll}5 & 13 & 52.96 & -68 & 18 & 37.2\end{array}$ & 1 & $(1,1)$ & 17.0 & (1) \\
\hline MG30 & $51414.69-705031.5$ & $\begin{array}{llllll}5 & 14 & 49.79 & -70 & 53 & 49.1\end{array}$ & 1 & $(1,1)$ & 18.5 & (1) \\
\hline MG31 & $\begin{array}{llllll}5 & 16 & 29.29 & -68 & 18 & 11.3\end{array}$ & $\begin{array}{llllll}5 & 16 & 40.69 & -68 & 21 & 20.2\end{array}$ & 1 & $(1,1)$ & 19.8 & (1) \\
\hline MG32 & $51904.04-645918.6$ & $\begin{array}{llllll}5 & 18 & 51.38 & -65 & 02 & 17.2\end{array}$ & 1 & $(1,1)$ & 19.0 & (1) \\
\hline MG33 & $\begin{array}{llllll}5 & 19 & 13.74 & -66 & 09 & 31.3\end{array}$ & $\begin{array}{llllll}5 & 19 & 08.97 & -66 & 12 & 28.9\end{array}$ & 1 & $(1,1)$ & 20.6 & (1) \\
\hline MG34 & $\begin{array}{lllll}5 & 1928.87 & -671426.5\end{array}$ & $\begin{array}{llllll}5 & 19 & 32.10 & -67 & 17 & 22.8\end{array}$ & 2 & $(3,1)$ & 20.0 & (3) \\
\hline MG35 & $51933.35-665537.2$ & $\begin{array}{llllll}5 & 19 & 34.21 & -66 & 58 & 33.2\end{array}$ & 2 & $(2,1)$ & 20.0 & $(2)$ \\
\hline MG36 & 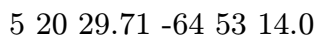 & $\begin{array}{llllll}5 & 20 & 16.55 & -64 & 56 & 06.4\end{array}$ & 1 & $(1,1)$ & 18.0 & (1) \\
\hline MG37 & $\begin{array}{llllll}5 & 21 & 46.81 & -65 & 22 & 26.7\end{array}$ & $\begin{array}{lllll}5 & 2136.95 & -65 & 25 & 13.5\end{array}$ & 2 & $(2,1)$ & 18.0 & $(2)$ \\
\hline MG38 & 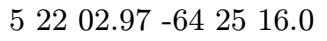 & 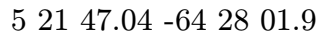 & 1 & $(1,1)$ & 18.8 & (1) \\
\hline MG39=LM2-19 & 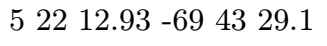 & $\begin{array}{llllll}5 & 22 & 37.85 & -69 & 46 & 12.8\end{array}$ & 1 & $(1,1)$ & 00.0 & $(0)$ \\
\hline MG40 & $\begin{array}{llllll}5 & 22 & 35.26 & -68 & 24 & 25.3\end{array}$ & $\begin{array}{llllll}5 & 22 & 48.30 & -68 & 27 & 07.9\end{array}$ & 1 & $(1,1)$ & 18.4 & (1) \\
\hline MG41 & $\begin{array}{llllll}5 & 23 & 37.73 & -65 & 09 & 54.3\end{array}$ & $\begin{array}{llllll}5 & 23 & 26.68 & -65 & 12 & 33.2\end{array}$ & 2 & $(2,1)$ & 19.2 & $(2)$ \\
\hline MG42 & 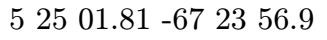 & $\begin{array}{llllll}5 & 25 & 06.88 & -67 & 26 & 29.2\end{array}$ & 2 & $(3,2)$ & 20.8 & (3) \\
\hline MG43 & $52434.19-711339.5$ & $\begin{array}{llllll}5 & 25 & 14.97 & -71 & 16 & 12.5\end{array}$ & 2 & $(2,1)$ & 19.5 & $(2)$ \\
\hline MG44 & $\begin{array}{lllll}5 & 25 & 55.67 & -6344 & 12.4\end{array}$ & $\begin{array}{llllll}5 & 25 & 35.95 & -63 & 46 & 41.7\end{array}$ & 1 & $(2,2)$ & 18.9 & $(2)$ \\
\hline MG45 & $\begin{array}{llllll}5 & 26 & 06.49 & -63 & 37 & 02.1\end{array}$ & $\begin{array}{llllll}5 & 25 & 46.08 & -63 & 39 & 30.6\end{array}$ & 1 & $(2,2)$ & 17.5 & $(2)$ \\
\hline MG46 & $\begin{array}{lllll}526 & 20.48 & -65 & 21 & 45.9\end{array}$ & $\begin{array}{llllll}5 & 26 & 10.99 & -65 & 24 & 13.0\end{array}$ & 2 & $(2,1)$ & 17.8 & $(2)$ \\
\hline MG47 & $\begin{array}{llllll}5 & 26 & 45.26 & -64 & 38 & 00.8\end{array}$ & $\begin{array}{llllll}5 & 26 & 31.08 & -64 & 40 & 26.3\end{array}$ & 1 & $(2,2)$ & 17.5 & $(2)$ \\
\hline MG48 & $\begin{array}{llllll}5 & 26 & 59.77 & -66 & 07 & 05.8\end{array}$ & $\begin{array}{llllll}5 & 26 & 55.51 & -66 & 09 & 29.9\end{array}$ & 1 & $(2,2)$ & 20.0 & $(2)$ \\
\hline MG49 & $\begin{array}{llllll}5 & 27 & 32.13 & -69 & 32 & 18.8\end{array}$ & $\begin{array}{llllll}5 & 27 & 55.87 & -69 & 34 & 39.5\end{array}$ & 1 & $(1,1)$ & 20.5 & (1) \\
\hline MG50 & 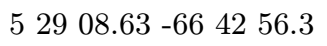 & 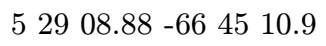 & 1 & $(2,2)$ & 19.2 & $(2)$ \\
\hline MG51 & $\begin{array}{llllll}5 & 28 & 34.41 & -703301.6\end{array}$ & 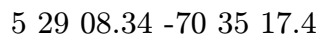 & 1 & $(2,2)$ & 17.5 & $(2)$ \\
\hline MG52 & $\begin{array}{lllll}5 & 29 & 08.78 & -69 & 4528.0\end{array}$ & $\begin{array}{llllll}5 & 29 & 34.79 & -69 & 47 & 41.7\end{array}$ & 1 & $(2,2)$ & 00.0 & $(0)$ \\
\hline MG53 & $\begin{array}{llllll}5 & 29 & 35.67 & -69 & 46 & 05.2\end{array}$ & $\begin{array}{llllll}5 & 30 & 01.83 & -69 & 48 & 16.9\end{array}$ & 2 & $(2,1)$ & 20.7 & (1) \\
\hline MG54 & $\begin{array}{llllll}5 & 29 & 51.41 & -68 & 50 & 05.6\end{array}$ & 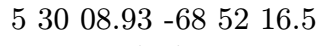 & 1 & $(2,2)$ & 19.2 & $(2)$ \\
\hline MG55 & $53109.07-713640.2$ & $53154.97-713844.5$ & 2 & $(2,1)$ & 17.7 & $(2)$ \\
\hline MG56 & $53145.00-694307.2$ & $\begin{array}{lllll}532 & 10.88 & -69 & 4509.6\end{array}$ & 2 & $(2,1)$ & 19.5 & $(2)$ \\
\hline MG57 & $53111.17-742253.3$ & $53234.92-742456.0$ & 1 & $(1,1)$ & 21.0 & (1) \\
\hline MG58 & $\begin{array}{llllll}5 & 32 & 37.68 & -67 & 08 & 31.1\end{array}$ & $\begin{array}{llllll}5 & 32 & 41.44 & -67 & 10 & 30.5\end{array}$ & 2 & $(4,3)$ & 19.2 & (4) \\
\hline MG59 & $53256.00-651642.0$ & $\begin{array}{llllll}5 & 32 & 46.47 & -65 & 18 & 40.5\end{array}$ & 1 & $(2,2)$ & 18.8 & $(2)$ \\
\hline MG60 & $\begin{array}{llllll}5 & 33 & 30.90 & -69 & 08 & 13.3\end{array}$ & $\begin{array}{llllll}5 & 33 & 51.47 & -69 & 10 & 08.2\end{array}$ & 2 & $(2,1)$ & 20.0 & $(2)$ \\
\hline MG61 & $\begin{array}{llllll}5 & 33 & 13.11 & -72 & 36 & 46.3\end{array}$ & $\begin{array}{llllll}5 & 34 & 11.55 & -72 & 38 & 41.1\end{array}$ & 1 & $(3,3)$ & 19.2 & $(3)$ \\
\hline MG62 & $53436.53-681827.9$ & $\begin{array}{llllll}5 & 34 & 49.87 & -68 & 20 & 18.3\end{array}$ & 1 & $(2,2)$ & 16.6 & (2) \\
\hline MG63 & $53410.21-714314.1$ & $\begin{array}{lllll}534 & 57.69 & -71 & 45 & 05.1\end{array}$ & 1 & $(2,2)$ & 18.5 & $(2)$ \\
\hline MG64 & $53512.77-673758.0$ & $\begin{array}{llllll}5 & 35 & 20.57 & -67 & 39 & 46.0\end{array}$ & 1 & $(4,4)$ & 20.1 & (4) \\
\hline MG65 & $\begin{array}{llllll}5 & 35 & 10.30 & -69 & 39 & 38.9\end{array}$ & 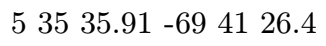 & 1 & $(2,2)$ & 19.9 & $(2)$ \\
\hline MG66 & $53759.54-655850.0$ & $\begin{array}{llllll}5 & 37 & 55.13 & -66 & 00 & 26.3\end{array}$ & 1 & $(1,1)$ & 19.7 & (1) \\
\hline MG67 & $53738.02-714137.9$ & $\begin{array}{llllll}5 & 38 & 25.47 & -71 & 43 & 14.0\end{array}$ & 1 & $(2,2)$ & 18.0 & $(2)$ \\
\hline MG68 & $\begin{array}{llllll}5 & 38 & 19.45 & -68 & 58 & 37.2\end{array}$ & $\begin{array}{llllll}5 & 38 & 38.92 & -69 & 00 & 11.2\end{array}$ & 1 & $(2,2)$ & 20.2 & $(2)$ \\
\hline MG69 & $53915.48-664943.5$ & $\begin{array}{llllll}5 & 39 & 17.30 & -66 & 51 & 14.1\end{array}$ & 2 & $(3,1)$ & 19.8 & $(3)$ \\
\hline MG70 & $\begin{array}{llllll}5 & 38 & 12.26 & -75 & 00 & 21.9\end{array}$ & $\begin{array}{llllll}5 & 39 & 47.17 & -75 & 01 & 53.7\end{array}$ & 1 & $(1,1)$ & 18.8 & (1) \\
\hline MG71 & $\begin{array}{llllll}5 & 39 & 54.87 & -66 & 34 & 13.1\end{array}$ & $\begin{array}{llllll}5 & 39 & 54.81 & -66 & 35 & 40.9\end{array}$ & 2 & $(2,1)$ & 19.7 & $(2)$ \\
\hline
\end{tabular}




\begin{tabular}{|c|c|c|c|c|c|c|}
\hline \multicolumn{7}{|c|}{ continued from previous page } \\
\hline \multirow[t]{2}{*}{ Name } & \multicolumn{2}{|c|}{ coord OPT } & \multirow{2}{*}{\multicolumn{2}{|c|}{$\mathrm{F} \quad(\mathrm{n} 1, \mathrm{n} 2)$}} & \multirow[t]{2}{*}{$m_{B}$} & \multirow[t]{2}{*}{$(\mathrm{n})$} \\
\hline & J2000 & B1950 & & & & \\
\hline MG72 & $\begin{array}{llllll} & 40 & 20.25 & -67 & 02 & 02.1\end{array}$ & $\begin{array}{llllll} & 40 & 23.69 & -67 & 03 & 27.9\end{array}$ & 2 & $(4,1)$ & 18.7 & $\overline{(4)}$ \\
\hline MG73 & $\begin{array}{llllll}5 & 41 & 36.61 & -69 & 27 & 09.8\end{array}$ & $\begin{array}{llllll}5 & 42 & 00.67 & -69 & 28 & 29.4\end{array}$ & 2 & $(2,1)$ & 19.7 & (2) \\
\hline MG74 & $54054.87-7441 \quad 19.2$ & 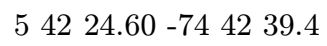 & 1 & $(1,1)$ & 17.2 & (1) \\
\hline MG75 & $542 \quad 15.41-684855.7$ & $\begin{array}{llllll}5 & 42 & 33.65 & -68 & 50 & 12.7\end{array}$ & 1 & $(2,2)$ & 19.1 & $(2)$ \\
\hline MG76 & $54224.24-695305.1$ & 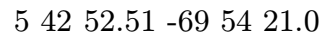 & 1 & $(2,2)$ & 15.6 & $(2)$ \\
\hline MG77 & $54347.59-683835.1$ & 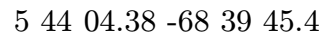 & 2 & $(2,1)$ & 19.8 & $(2)$ \\
\hline MG78 & $\begin{array}{llllll}5 & 4625.41 & -6750 & 02.8\end{array}$ & $\begin{array}{llllll}5 & 46 & 35.46 & -67 & 51 & 01.9\end{array}$ & 1 & $(2,2)$ & 20.9 & $(2)$ \\
\hline MG79 & $54559.68-711904.9$ & $\begin{array}{llllll}5 & 46 & 43.30 & -71 & 20 & 04.7\end{array}$ & 2 & $(2,1)$ & 17.1 & $(2)$ \\
\hline MG80 & $54929.11-675454.1$ & $\begin{array}{llllll}5 & 49 & 39.92 & -67 & 55 & 39.8\end{array}$ & 1 & $(2,2)$ & 19.6 & $(2)$ \\
\hline MG81 & $55100.09-675845.6$ & 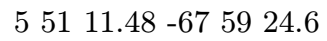 & 1 & $(2,2)$ & 20.1 & (2) \\
\hline MG82 & $\begin{array}{llllll}5 & 53 & 11.87 & -71 & 28 & 57.0\end{array}$ & $55357.59-712925.2$ & 1 & $(1,1)$ & 18.9 & (1) \\
\hline MG83 & $\begin{array}{llllll}5 & 54 & 39.41 & -69 & 20 & 34.6\end{array}$ & $\begin{array}{llllll}5 & 55 & 02.88 & -69 & 20 & 57.2\end{array}$ & 1 & $(1,1)$ & 16.9 & (1) \\
\hline MG84 & $\begin{array}{lllll}5 & 54 & 41.47 & -72 & 23 \\
31.8\end{array}$ & $\begin{array}{lllll}5 & 5538.26 & -72 & 23 & 53.0\end{array}$ & 1 & $(2,2)$ & 19.2 & (2) \\
\hline MG85 & $60410.14-700422.9$ & 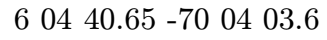 & 1 & $(1,1)$ & 19.2 & (1) \\
\hline MG86 & $\begin{array}{lllll}6 & 0458.70-720353.0\end{array}$ & $\begin{array}{llllll}6 & 05 & 51.30 & -72 & 03 & 29.4\end{array}$ & 1 & $(2,2)$ & 20.1 & $(2)$ \\
\hline Mo01 & $43451.80-681602.8$ & $43454.65-682206.6$ & 1 & $(1,1)$ & 00.0 & $(0)$ \\
\hline $\mathrm{Mo02}$ & $43446.67-692319.6$ & $43458.91-692923.5$ & 1 & $(1,1)$ & 00.0 & (0) \\
\hline Mo03 & $43614.41-703103.0$ & $43637.60-703700.5$ & 3 & $(2,1)$ & 20.1 & (1) \\
\hline $\mathrm{Mo04}$ & $44012.30-645532.6$ & $43953.04-650115.3$ & 1 & $(1,1)$ & 19.7 & (1) \\
\hline Mo05 & $44519.91-673433.4$ & $\begin{array}{lllll}4 & 45 & 19.90 & -67 & 39 \\
54.3\end{array}$ & 1 & $(3,2)$ & 20.3 & $(2)$ \\
\hline Mo06 & $44633.07-701246.4$ & $\begin{array}{llllll}4 & 46 & 56.06 & -70 & 18 & 01.4\end{array}$ & 1 & $(2,2)$ & 20.1 & (1) \\
\hline Mo07 & $44919.86-642235.7$ & 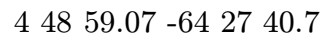 & 1 & $(1,1)$ & 19.5 & (1) \\
\hline Mo08 & $45024.35-642151.1$ & $45003.68-642651.6$ & 1 & $(1,1)$ & 19.4 & (1) \\
\hline Mo09 & $45211.39-701246.4$ & 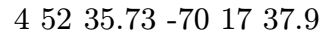 & 1 & $(1,1)$ & 18.2 & (1) \\
\hline Mo10 & $45545.43-654834.7$ & $\begin{array}{lllll}45534.86 & -65 & 53 & 12.4\end{array}$ & 1 & $(1,1)$ & 19.7 & (1) \\
\hline Mo11 & 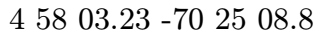 & $45830.93-702935.6$ & 1 & $(1,1)$ & 19.9 & (1) \\
\hline Mo12 & $45837.00-693546.5$ & 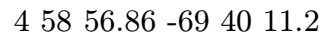 & 1 & $(1,1)$ & 19.7 & (1) \\
\hline Mo13 & $45909.07-695030.8$ & $45931.35-695453.1$ & 1 & $(1,1)$ & 20.2 & (1) \\
\hline Mo14 & $\begin{array}{lllll}5 & 0136.50-702631.9\end{array}$ & $\begin{array}{llllll}5 & 02 & 05.17 & -70 & 30 & 43.6\end{array}$ & 1 & $(1,1)$ & 19.5 & (1) \\
\hline Mo15 & 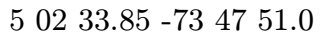 & $\begin{array}{lllll}5 & 03 & 43.52 & -73 & 5157.2\end{array}$ & 1: & $(1,1)$ & 00.0 & (0) \\
\hline Mo16 & $\begin{array}{llllll}5 & 06 & 14.93 & -69 & 48 & 24.7\end{array}$ & $\begin{array}{llllll}5 & 06 & 38.22 & -69 & 52 & 16.9\end{array}$ & 1 & $(1,1)$ & 20.2 & (1) \\
\hline Mo17 & 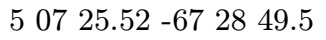 & $\begin{array}{llllll}5 & 07 & 28.96 & -67 & 32 & 37.4\end{array}$ & 2 & $(2,1)$ & 20.2 & $(2)$ \\
\hline Mo18 & $\begin{array}{llllll}5 & 09 & 11.19 & -673401.7\end{array}$ & $\begin{array}{llllll}5 & 09 & 15.57 & -67 & 37 & 42.1\end{array}$ & 1 & $(2,2)$ & 17.7 & $(2)$ \\
\hline Mo19 & $\begin{array}{llllll}5 & 11 & 00.46 & -70 & 0504.8\end{array}$ & $\begin{array}{llllll}5 & 11 & 27.27 & -70 & 08 & 36.5\end{array}$ & 1 & $(1,1)$ & 18.0 & (1) \\
\hline Mo20 & 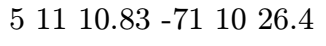 & $\begin{array}{llllll}5 & 11 & 49.02 & -71 & 13 & 57.0\end{array}$ & 1 & $(1,1)$ & 20.1 & (1) \\
\hline Mo21 & $\begin{array}{llllll}5 & 19 & 04.10 & -64 & 44 & 38.1\end{array}$ & $\begin{array}{llllll}5 & 18 & 49.87 & -64 & 47 & 36.8\end{array}$ & 1 & $(1,1)$ & 20.9 & (1) \\
\hline Mo22 & $52126.64-623412.5$ & $\begin{array}{llllll}5 & 20 & 59.92 & -62 & 37 & 01.3\end{array}$ & 1 & $(1,1)$ & 17.9 & (1) \\
\hline Mo23 & 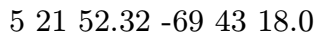 & $\begin{array}{llllll}5 & 22 & 17.17 & -69 & 46 & 03.1\end{array}$ & 1: & $(1,1)$ & 00.0 & (0) \\
\hline Mo24 & $\begin{array}{llllll}5 & 22 & 53.20 & -71 & 05 & 40.7\end{array}$ & $\begin{array}{llllll}5 & 23 & 32.29 & -71 & 08 & 21.0\end{array}$ & 1 & $(1,1)$ & 19.2 & (1) \\
\hline Mo25 & $\begin{array}{llllll}5 & 26 & 02.56 & -72 & 31 & 02.5\end{array}$ & $\begin{array}{llll}526 & 58.96 & -72 & 3328.5\end{array}$ & 2 & $(3,2)$ & 20.2 & (3) \\
\hline Mo26 & $\begin{array}{llllll}5 & 28 & 01.52 & -70 & 13 & 30.1\end{array}$ & $\begin{array}{llllll}5 & 28 & 32.04 & -70 & 15 & 48.4\end{array}$ & 2 & $(2,1)$ & 19.8 & $(2)$ \\
\hline Mo27 & $\begin{array}{llllll}5 & 29 & 16.65 & -69 & 37 & 18.1\end{array}$ & $\begin{array}{lllll}5 & 29 & 41.36 & -69 & 39 \\
\end{array}$ & 1 & $(2,2)$ & 19.9 & (1) \\
\hline Mo28 & $\begin{array}{llllll}5 & 29 & 18.43 & -70 & 23 & 49.7\end{array}$ & 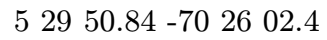 & 1 & $(2,2)$ & 18.6 & (2) \\
\hline Mo29 & 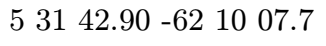 & $\begin{array}{llllll}5 & 31 & 14.82 & -62 & 12 & 12.2\end{array}$ & 1 & $(2,2)$ & 20.8 & $(2)$ \\
\hline Mo30 & $\begin{array}{lllll}5 & 3135.30 & -69 & 23 & 46.4\end{array}$ & $53158.10-692549.6$ & 2 & $(2,1)$ & 18.8 & $(2)$ \\
\hline Mo31 & $\begin{array}{llllll}5 & 32 & 43.37 & -62 & 32 & 39.7\end{array}$ & $\begin{array}{llllll}5 & 32 & 17.38 & -62 & 34 & 39.7\end{array}$ & 2 & $(2,1)$ & 18.4 & $(2)$ \\
\hline Mo32=?LM2-33 & 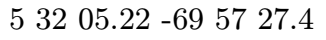 & 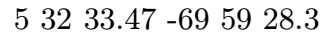 & 2 & $(2,1)$ & 20.6 & (1) \\
\hline Mo33 & $53209.28-702441.5$ & $\begin{array}{llllll}5 & 32 & 42.12 & -70 & 26 & 41.8\end{array}$ & 2 & $(2,1)$ & 17.9 & $(2)$ \\
\hline Mo34 & $\begin{array}{llllll}5 & 35 & 13.81 & -70 & 01 & 19.2\end{array}$ & 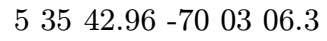 & 1 & $(2,2)$ & 18.9 & $(2)$ \\
\hline Mo35 & $\begin{array}{llllll}5 & 38 & 04.85 & -70 & 29 & 25.8\end{array}$ & $\begin{array}{lllllll}5 & 38 & 39.00 & -70 & 31 & 00.4\end{array}$ & 1 & $(2,2)$ & 18.7 & (2) \\
\hline Mo36 & $53853.56-695755.7$ & $\begin{array}{lllll}5 & 39 & 22.42 & -69 & 59 \\
26.9\end{array}$ & 1 & $(2,2)$ & 19.3 & $(2)$ \\
\hline Mo37 & $\begin{array}{llllll}5 & 39 & 14.47 & -70 & 00 & 18.6\end{array}$ & $\begin{array}{llllll}5 & 39 & 43.75 & -70 & 01 & 48.3\end{array}$ & 2 & $(2,2)$ & 20.8 & $(2)$ \\
\hline
\end{tabular}




\begin{tabular}{|c|c|c|c|c|c|c|}
\hline \multicolumn{7}{|c|}{ continued from previous page } \\
\hline \multirow[t]{2}{*}{ Name } & \multicolumn{2}{|c|}{ coord OPT } & \multirow{2}{*}{\multicolumn{2}{|c|}{$\begin{array}{ll}\mathrm{F} & (\mathrm{n} 1, \mathrm{n} 2)\end{array}$}} & \multirow[t]{2}{*}{$m_{B}$} & \multirow[t]{2}{*}{ (n) } \\
\hline & J2000 & B1950 & & & & \\
\hline Mo38 & $54032.26-6844 \quad 47.6$ & $\begin{array}{llllll}5 & 40 & 49.80 & -68 & 46 & 12.1\end{array}$ & 2 & $\overline{(2,1)}$ & 20.6 & $(2)$ \\
\hline Mo39 & $\begin{array}{llllll}5 & 42 & 41.09 & -70 & 05 & 49.1\end{array}$ & $\begin{array}{llllll}5 & 43 & 11.49 & -70 & 07 & 03.7\end{array}$ & 2 & $(2,1)$ & 20.4 & (2) \\
\hline Mo40 & 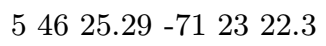 & $54709.74-712420.1$ & 2 & $(2,1)$ & 19.7 & $(2)$ \\
\hline Mo41 & $\begin{array}{llllll}5 & 55 & 13.70 & -65 & 28 & 22.8\end{array}$ & $\begin{array}{llllll}5 & 55 & 06.37 & -65 & 28 & 44.0\end{array}$ & 1 & $(1,1)$ & 19.2 & (1) \\
\hline Mo42 & $\begin{array}{lllll}5 & 5514.61 & -66 & 5024.6\end{array}$ & $\begin{array}{llllll}5 & 55 & 17.05 & -66 & 50 & 45.4\end{array}$ & 1 & $(1,1)$ & 18.8 & (1) \\
\hline Mo43 & $55846.10-643045.0$ & $55832.52-643050.9$ & 1 & $(1,1)$ & 17.9 & (1) \\
\hline Mo44 & $\begin{array}{llllll}6 & 01 & 43.34 & -68 & 00 & 35.0\end{array}$ & $\begin{array}{llllll}6 & 01 & 55.04 & -68 & 00 & 27.1\end{array}$ & 1 & $(2,2)$ & 19.1 & (2) \\
\hline Mo45 & $60917.13-684352.2$ & $60934.84-684311.0$ & 1 & $(1,1)$ & 18.3 & (1) \\
\hline Mo46 & $\begin{array}{llllll}6 & 10 & 09.61 & -67 & 10 & 19.2\end{array}$ & $\begin{array}{llllll}6 & 10 & 14.40 & -67 & 09 & 34.7\end{array}$ & 1 & $(3,2)$ & 19.8 & (2) \\
\hline Mo47 & $\begin{array}{llllll}6 & 13 & 03.38 & -65 & 55 & 09.3\end{array}$ & $61258.84-655412.5$ & 1 & $(2,2)$ & 20.3 & (1) \\
\hline Mo48 & $\begin{array}{llllll}6 & 13 & 41.12 & -64 & 00 & 45.3\end{array}$ & $61324.15-635946.2$ & 1 & $(2,2)$ & 18.0 & (1) \\
\hline Mo49=?LM1-64 & $\begin{array}{llllll}6 & 21 & 04.79 & -66 & 08 & 00.4\end{array}$ & $62101.34-660628.5$ & 1 & $(2,1)$ & 20.3 & (1) \\
\hline Mo50 & $62114.99-685745.6$ & $62134.10-685612.2$ & 1 & $(1,1)$ & 19.5 & (1) \\
\hline Mo51 & $\begin{array}{llllll}6 & 25 & 04.38 & -69 & 07 & 36.9\end{array}$ & 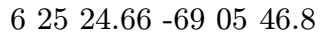 & 1 & $(1,1)$ & 19.4 & (1) \\
\hline Mo52 & $\begin{array}{llllll}6 & 26 & 35.98 & -69 & 49 & 38.2\end{array}$ & $62702.71-694741.2$ & 1 & $(1,1)$ & 19.8 & (1) \\
\hline Mo53 & $63818.64-692417.3$ & $63839.99-692129.8$ & 1 & $(1,1)$ & 18.8 & (1) \\
\hline Mo54 & $\begin{array}{llllll}6 & 41 & 24.62 & -67 & 01 & 29.2\end{array}$ & $\begin{array}{llllll}6 & 41 & 25.72 & -66 & 58 & 29.1\end{array}$ & 1 & $(1,1)$ & 00.0 & $(0)$ \\
\hline $\mathrm{SMP} 026=\mathrm{N} 101$ & $\begin{array}{llllll}5 & 07 & 30.77 & -69 & 08 & 07.0\end{array}$ & $\begin{array}{lllll}5 & 07 & 48.06 & -69 & 1154.0\end{array}$ & 1 & $(1,1)$ & 19.4 & (1) \\
\hline \multicolumn{7}{|l|}{ Denomination } \\
\hline \multicolumn{7}{|c|}{ BE : Bohannan \& Epps (1974) } \\
\hline \multicolumn{7}{|c|}{$\mathrm{J}:$ Jacoby $(1980)$} \\
\hline \multicolumn{7}{|c|}{ L : Lindsay (1963) } \\
\hline \multicolumn{7}{|c|}{ LM1 : Lindsay \& Mullan (1963) } \\
\hline \multicolumn{7}{|c|}{ LM2 : Lindsay \& Mullan (1963) } \\
\hline \multicolumn{7}{|c|}{ MG : Morgan \& Good (1992) } \\
\hline \multicolumn{7}{|c|}{ Mo : Morgan (1994) } \\
\hline \multicolumn{7}{|c|}{ N : nebulae Henize (1956) } \\
\hline \multicolumn{7}{|c|}{ S : stars Henize (1956) } \\
\hline \multicolumn{7}{|c|}{ SMC1 : Savage, Murdin, Clark (1982) } \\
\hline \multicolumn{7}{|c|}{ SMP: Sanduleak et al. (1978) } \\
\hline \multicolumn{7}{|c|}{ Sa: Sanduleak (1984) } \\
\hline WS : Westerlund & Smith & & & & & \\
\hline
\end{tabular}

Table 4. LMC positions 


\section{References}

Alard C., 1996, PhD Thesis, Université de Paris 6 Alcaino G., 1975, A\&AS 21, 279

Berger J., Cordoni J.P., Fringant A.M., Guibert J., Moreau O., Reboul H., Vanderriest C., 1991, A\&AS 87, 389

Blair M., Gilmore G., 1982, PASP 94, 742

Boroson T.A., Liebert J., 1989, ApJ 339, 844

Dennefeld M., 1986, A\&A 160, 199

Fehrenbach Ch., Duflot M., Acker A., 1978, A\&AS 33, 115

Henize K.G., 1956, ApJS 2, 315

Jacoby G.H., 1980, ApJS 42, 1

Jacoby G.H., Walker A.R, Ciardullo R., 1990, ApJ 365, 471

Leisy P., Dennefeld M., 1996, A\&AS 116, 95

Lieske J.H., 1979, A\&A 73, 282

Linde P., Ardeberg A., Lindgren H., Lyngå G., 1988, A\&AS 73,37

Lindsay E.M., 1963, Ir. Astron. J. 6, 127

Lindsay E.M., Mullan A.D.J., 1963, Ir. Astron. J. 6, 51

Meatheringham S.J., Dopita M.A., 1991a, ApJS 75, 407

Meatheringham S.J, Dopita M.A., 1991b, ApJS 76, 1085
Morgan D.H., Good A.R., 1992, A\&AS 92, 571

Morgan D.H., 1994, A\&AS 103, 235

Pottasch S.R., Bignell C., Olling R., Zijlstra A.A., 1988, A\&A 205, 248

Röser S., Bastien U., 1988, A\&AS 74, 449

Sanduleak N., Phillip A.G.D., 1977, PASP 89, 792

Sanduleak N., MacConnell D.J., Phillip A.G.D., 1978, PASP 90,621

Sanduleak N., 1984, I.A.U 108, 231

Savage A., Murdin P.G., Clarck D.H., 1982, Observatory 102, 229

Schwan H., 1988, A\&A 198, 363

Schwering P.B.W., Israel F.P., 1990. Kluwer Academic Publishers Dordrecht, Holland

Vassiliadis E., Dopita M.A., Morgan D.H., Bell J.F., 1992, ApJ 83,087

Westerlund B.E., Smith L.F., 1964, MNRAS 127, 449

Zijlstra A.A., Van Hoof P.A.M., Chapman J.M., Loup C., 1994, A\&A 290, 228 
$N$

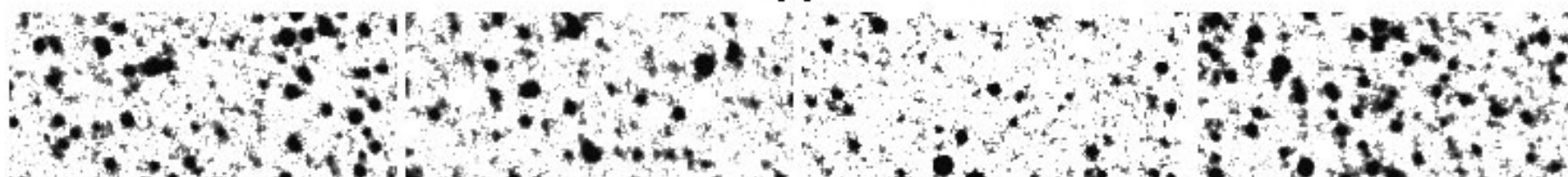

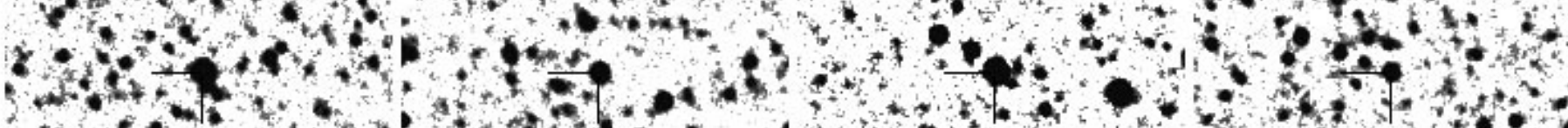

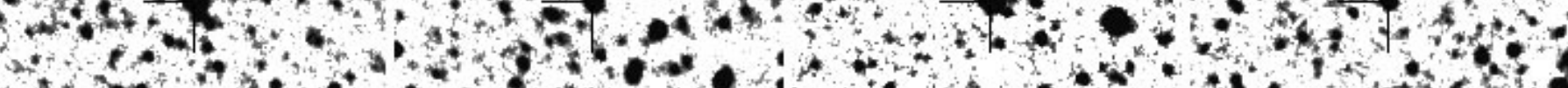

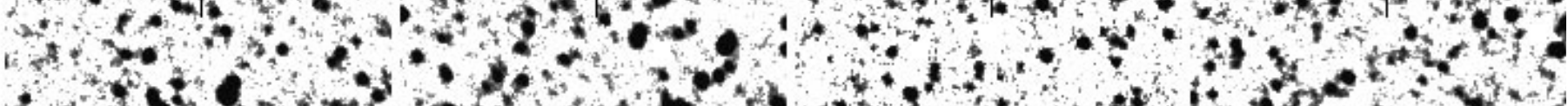

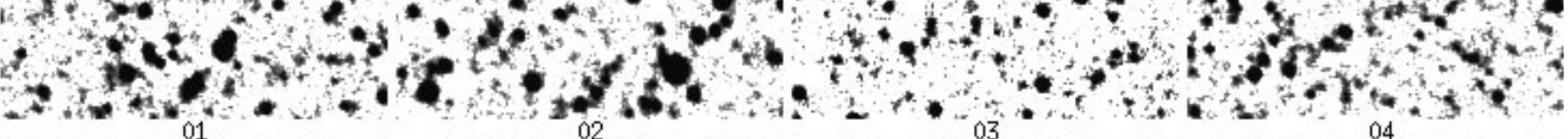

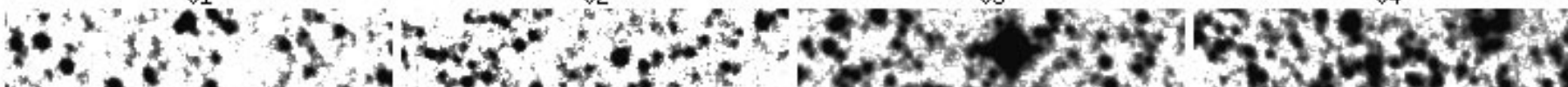

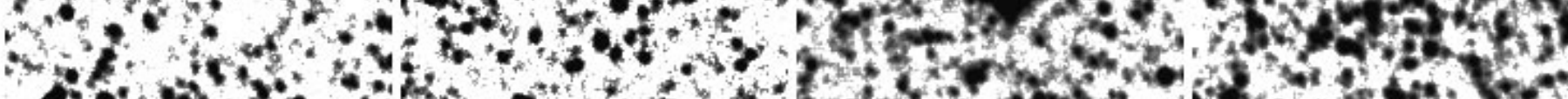

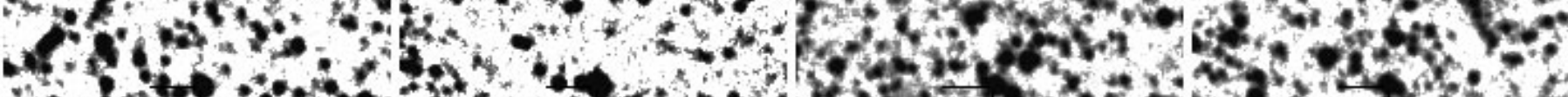

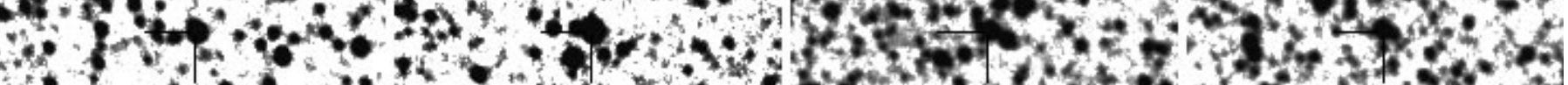

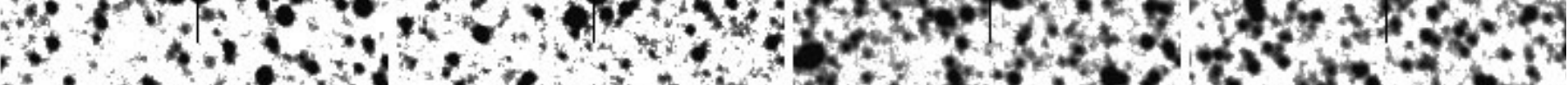

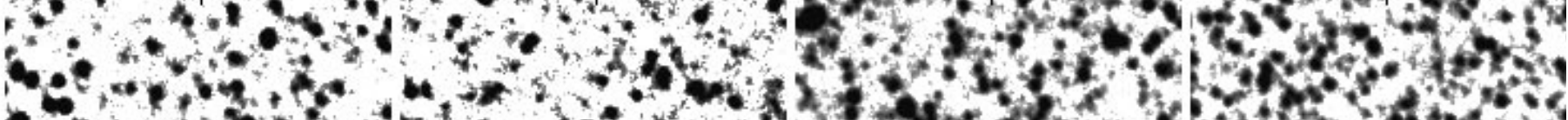

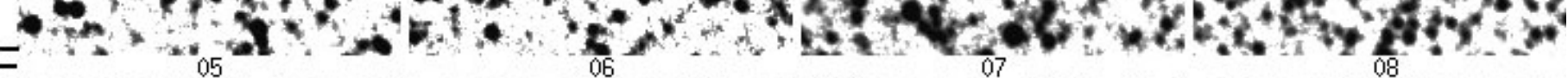

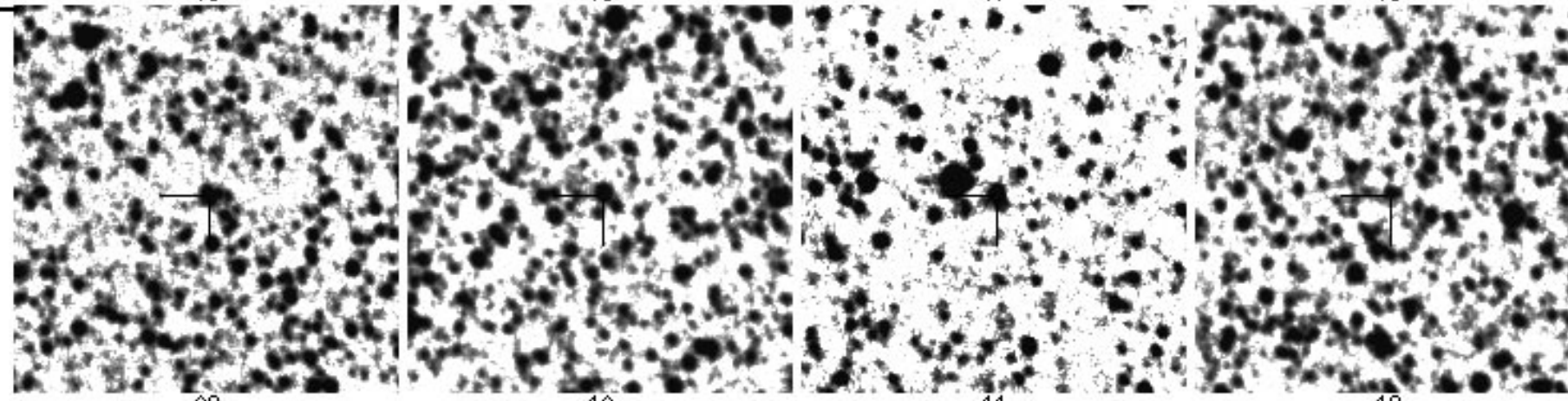

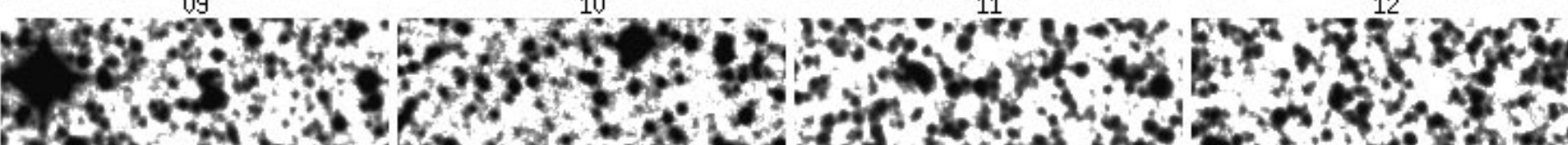

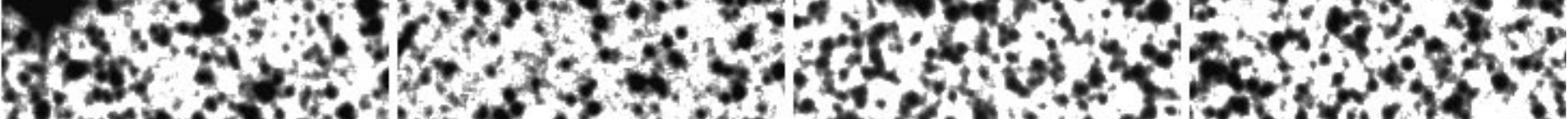

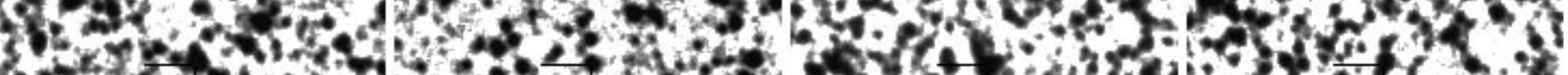
a.t. r. Whis

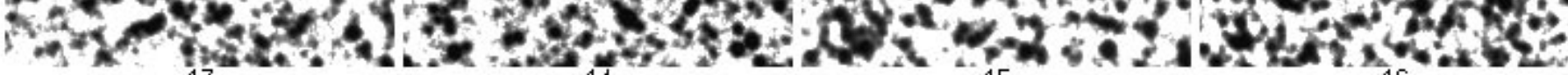

Fig. 7. Finding charts Sanduleak et al. 
$\mathrm{N}$

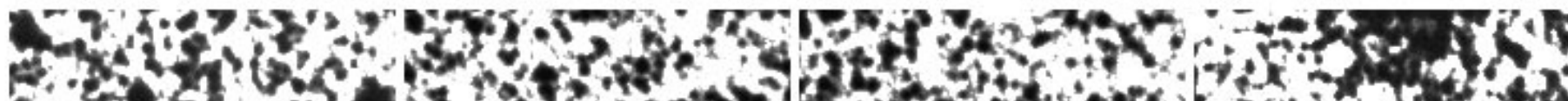

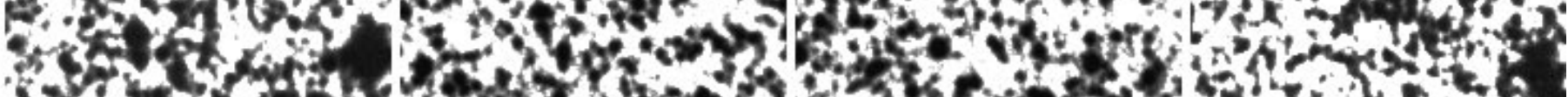

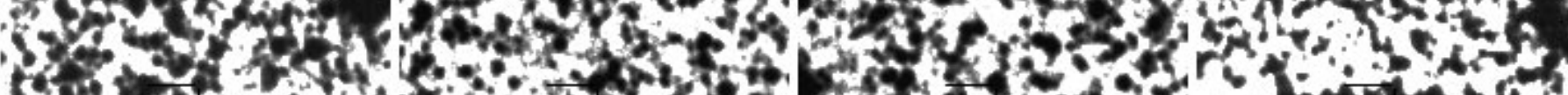

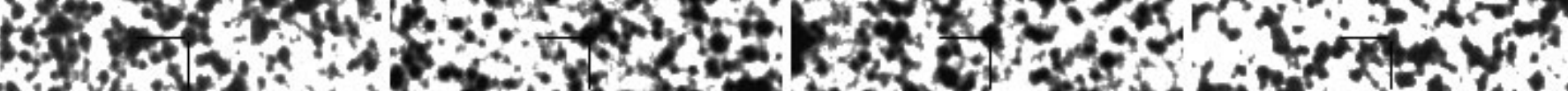

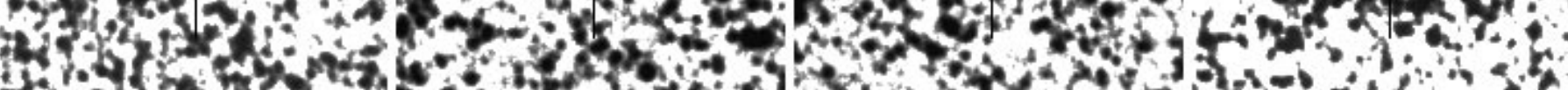

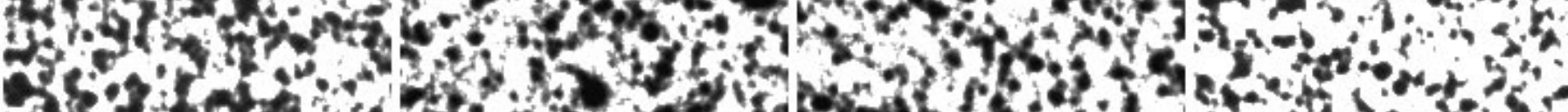

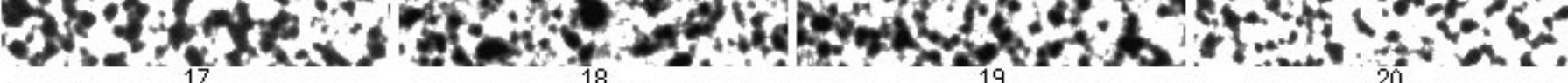

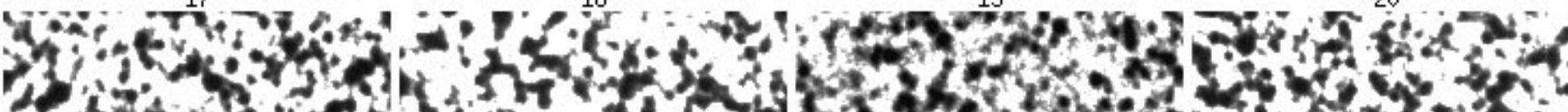

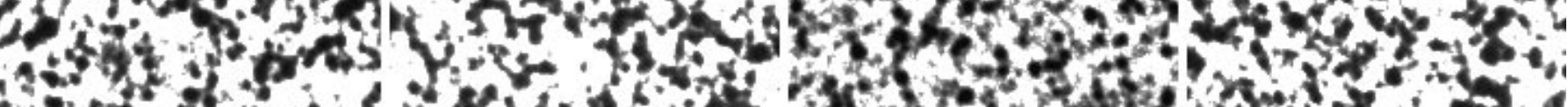

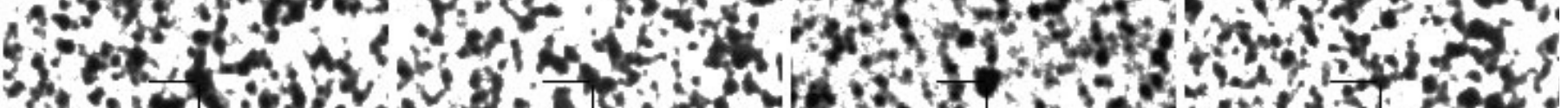

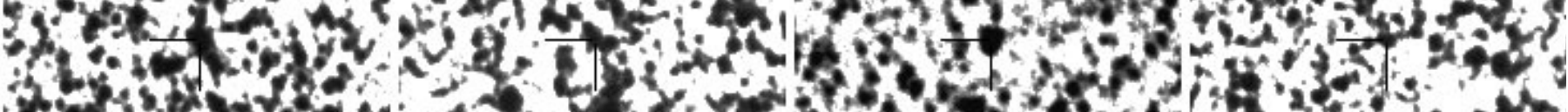

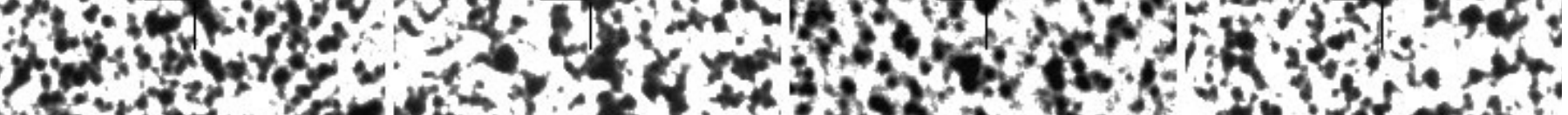

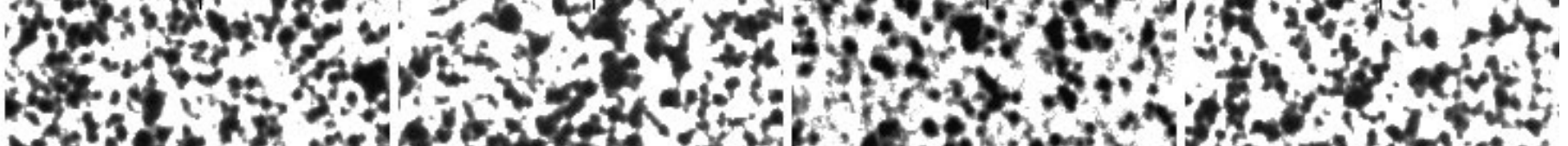
=

IN W

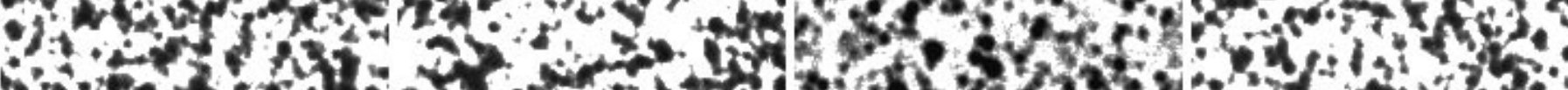

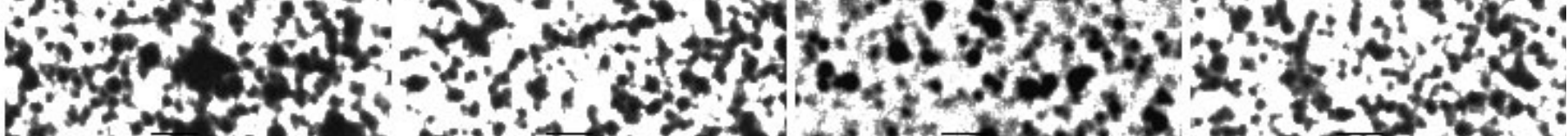

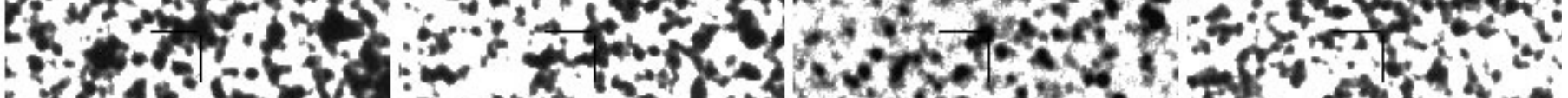

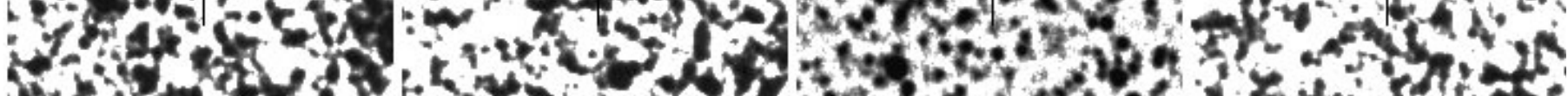

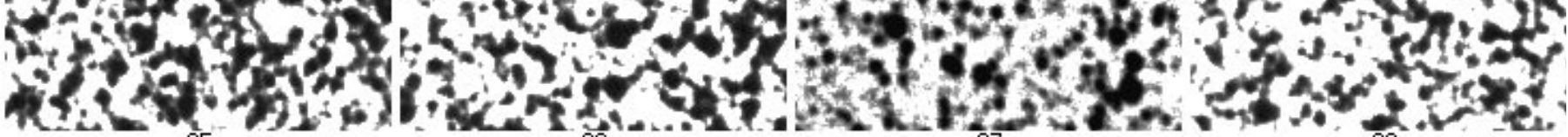

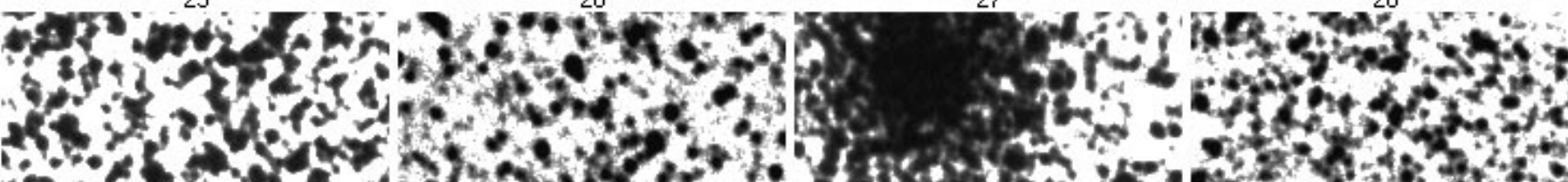

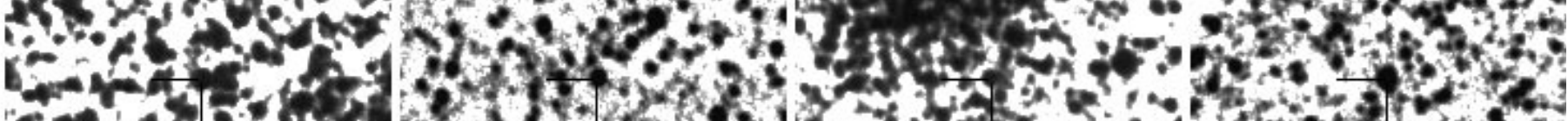

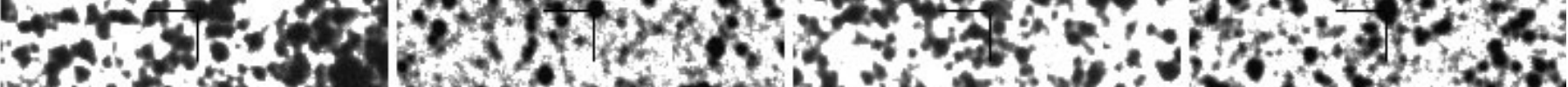

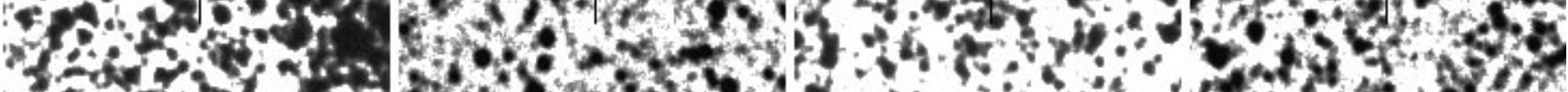

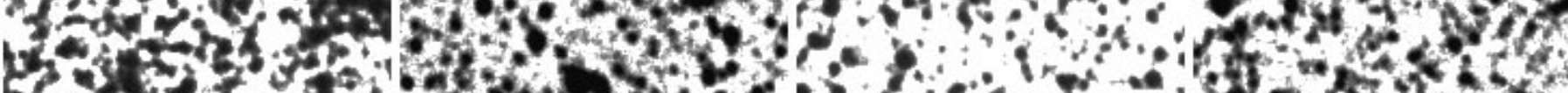

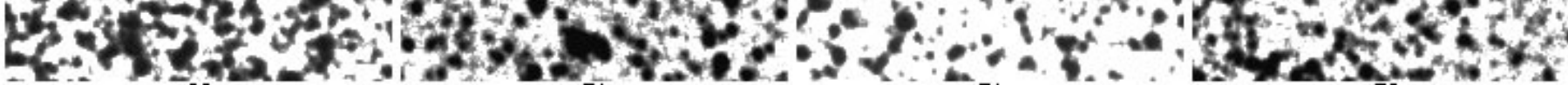
29

Fig. 8. Finding charts Sanduleak et al. 
$\mathrm{N}$

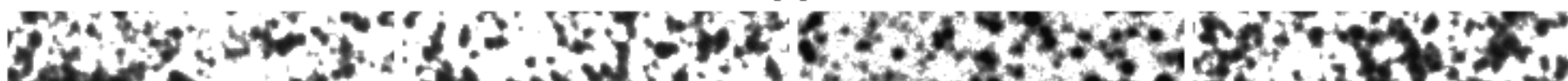

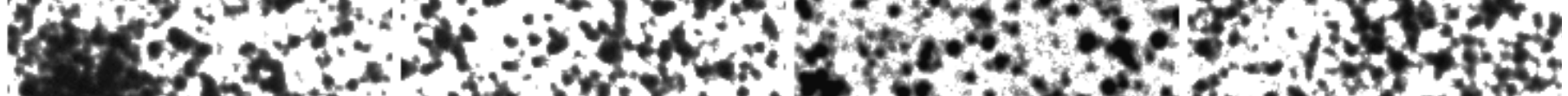

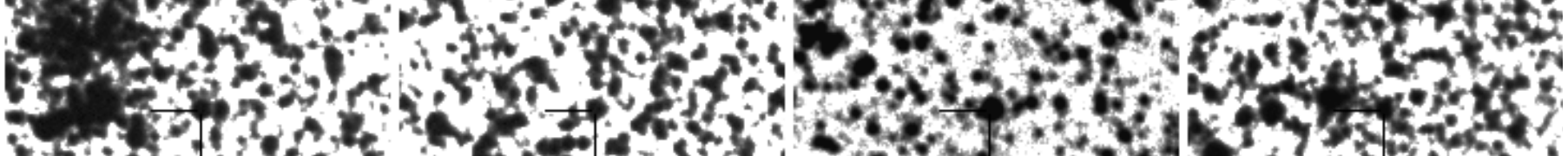

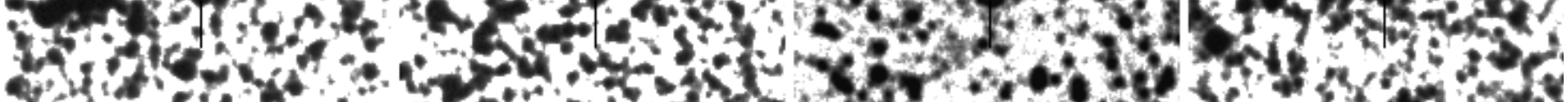
S. Lid ,

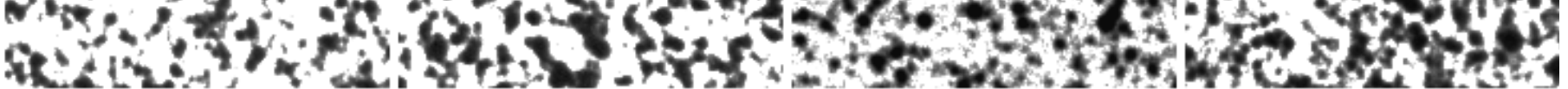

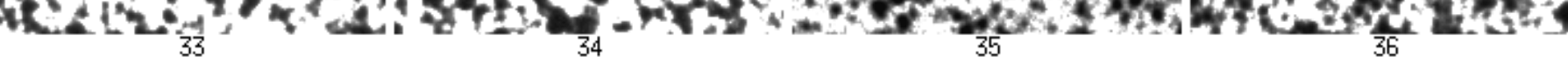
w.

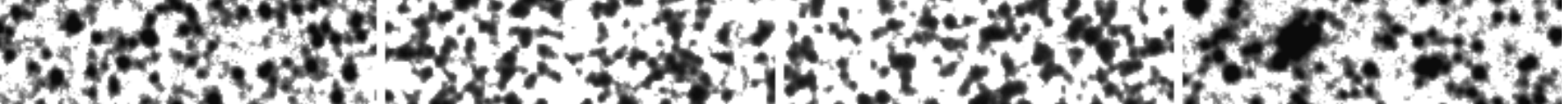
1010.

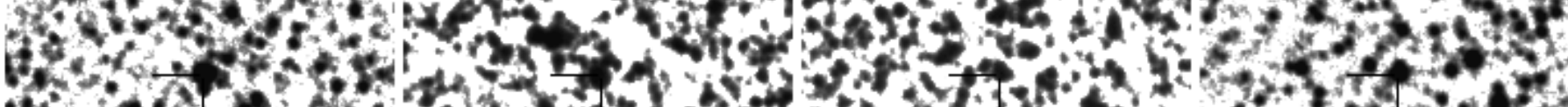

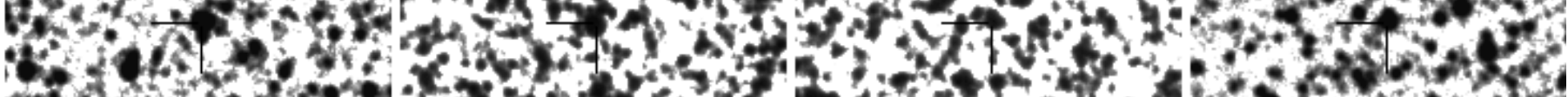

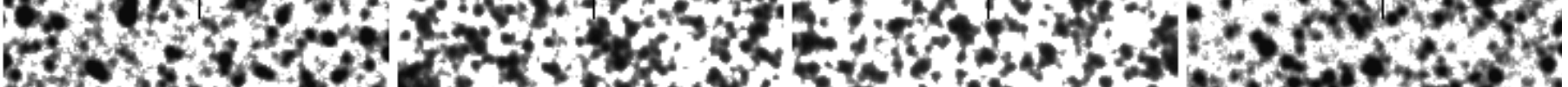

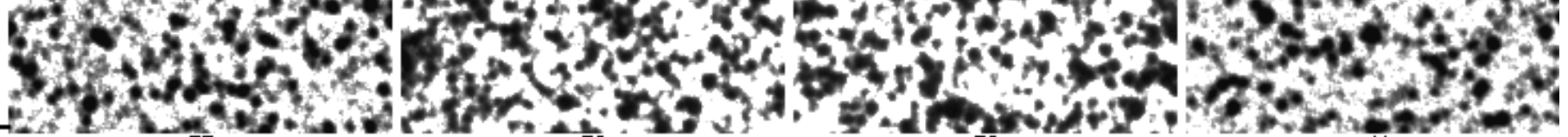

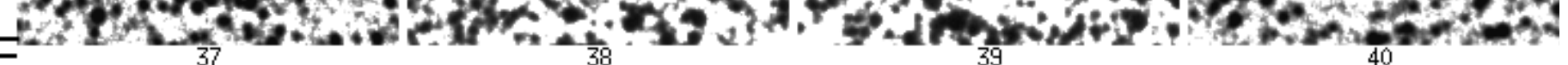

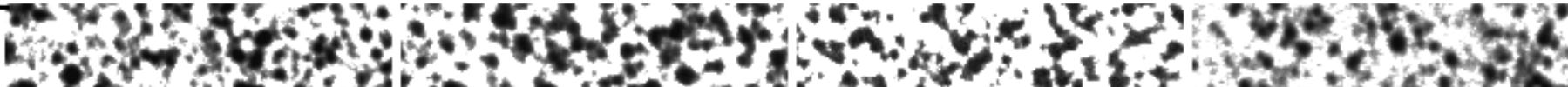

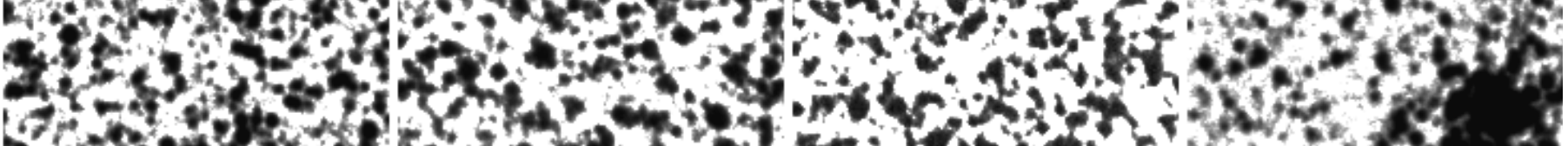

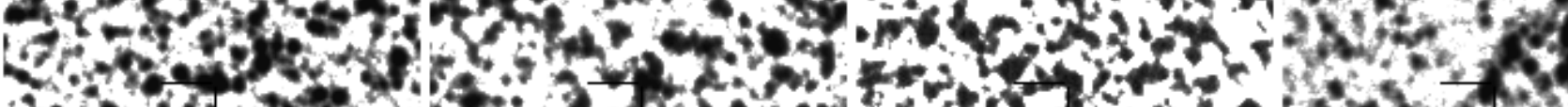

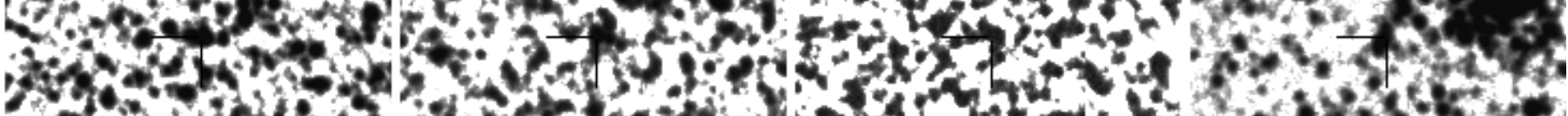
Hof

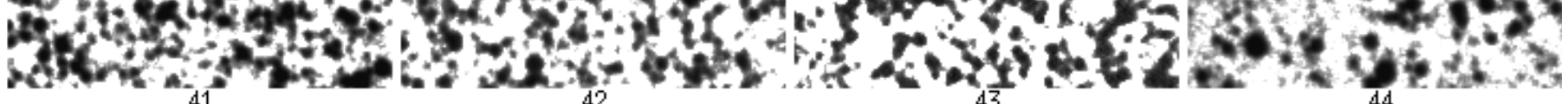

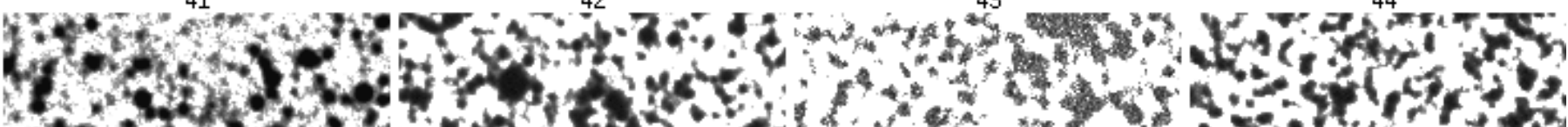

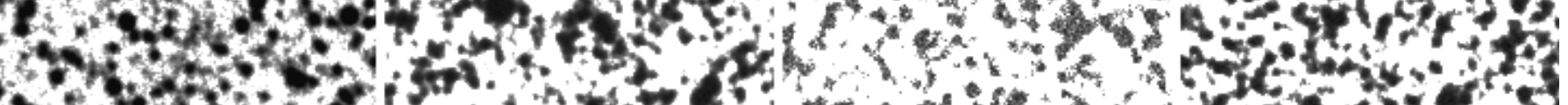

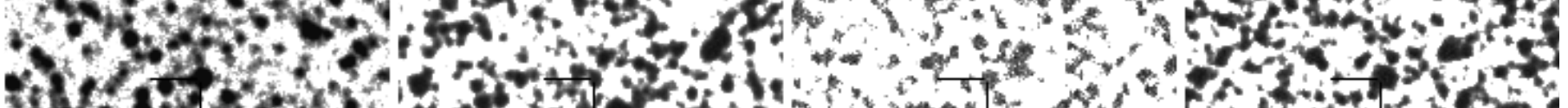

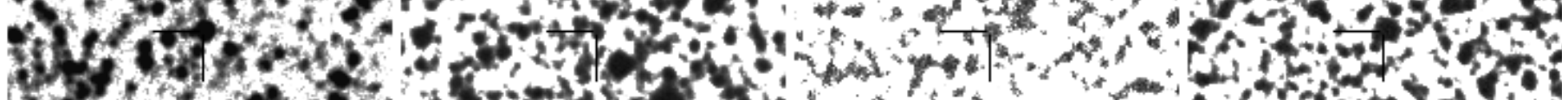

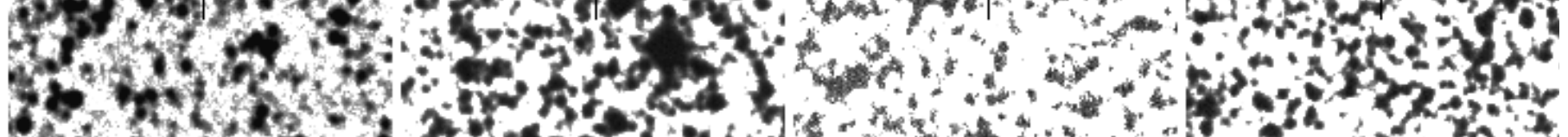

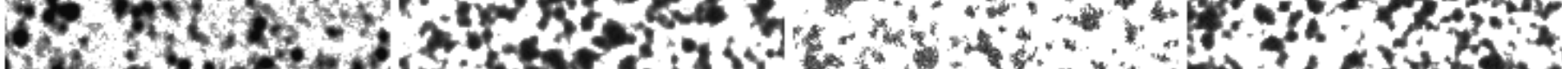

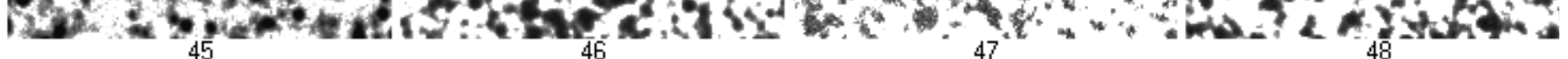


$\mathrm{N}$

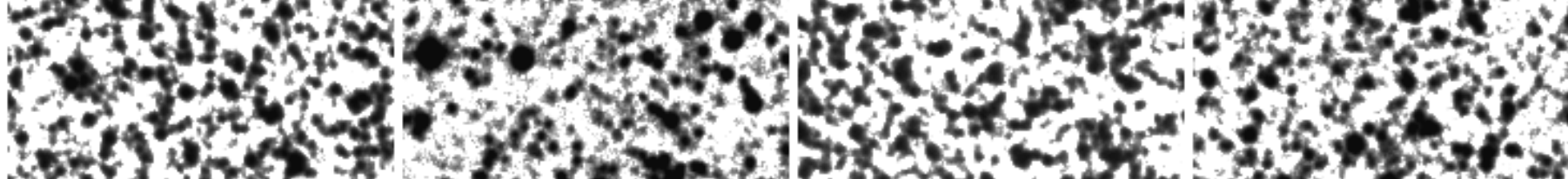

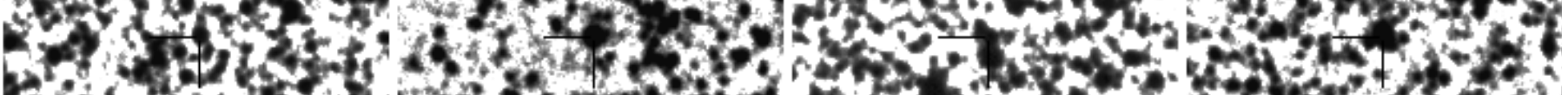
b.

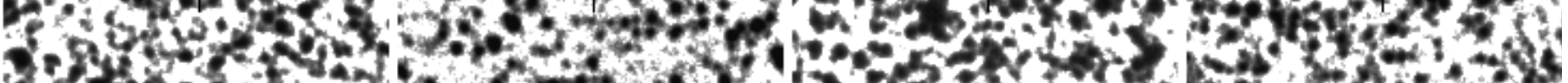
3.

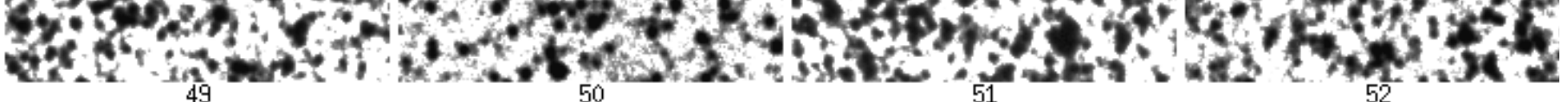

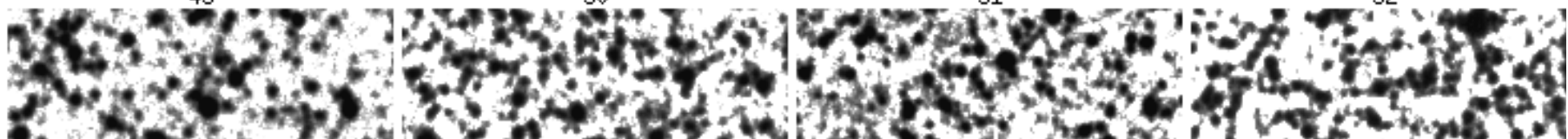

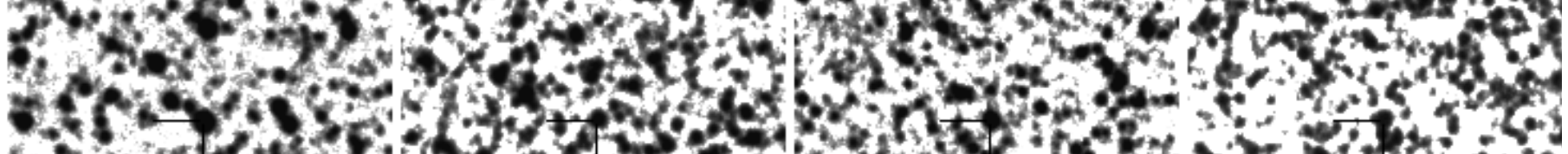

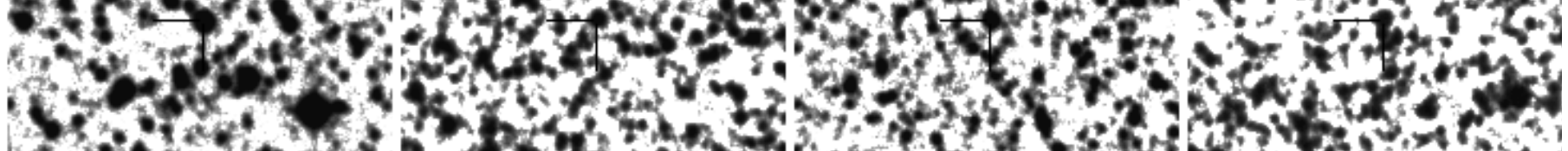

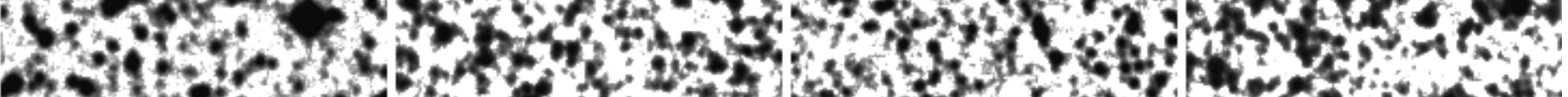

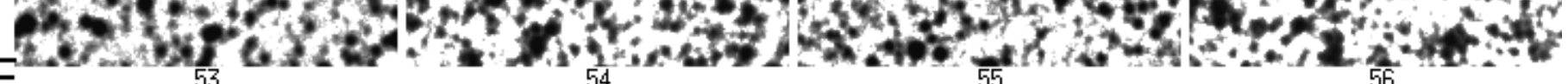

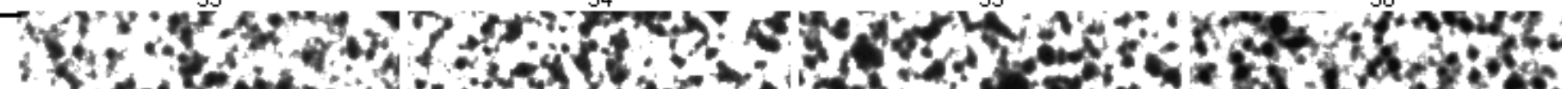

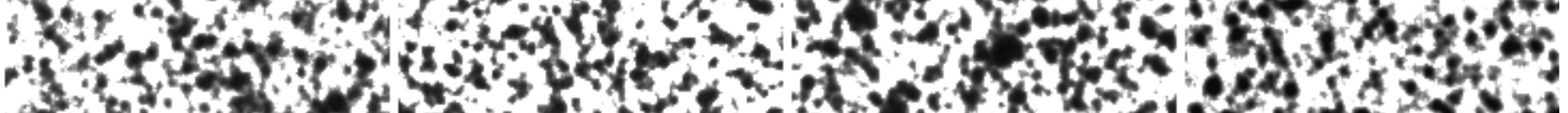

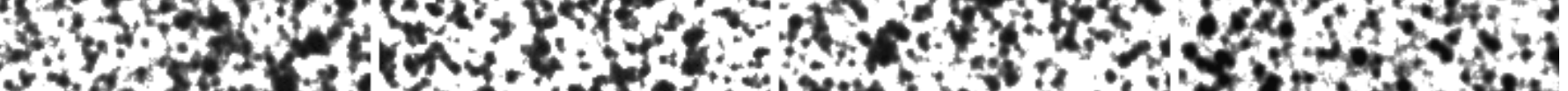

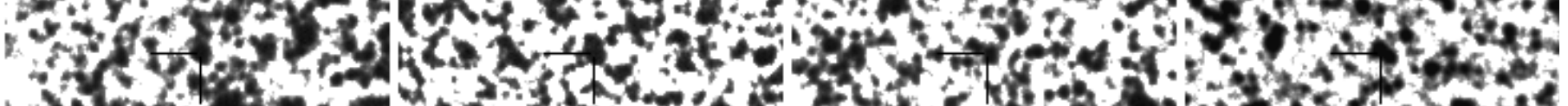

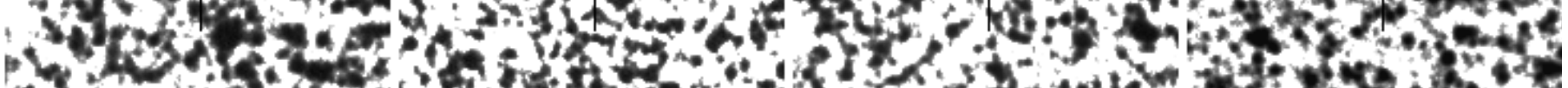

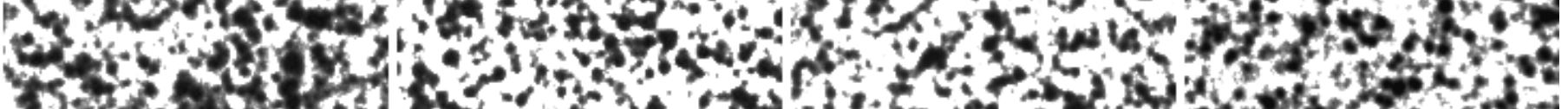

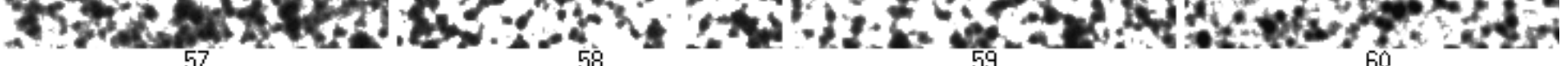
5to

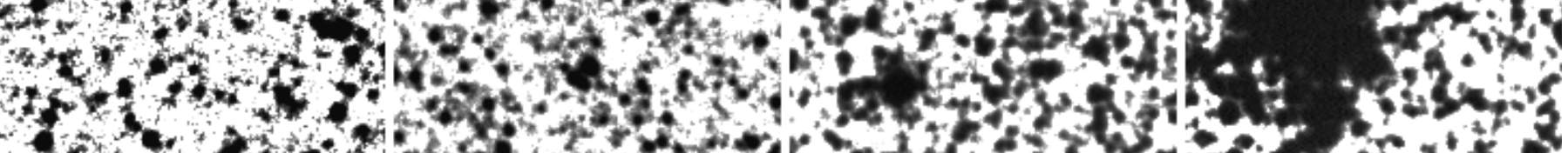

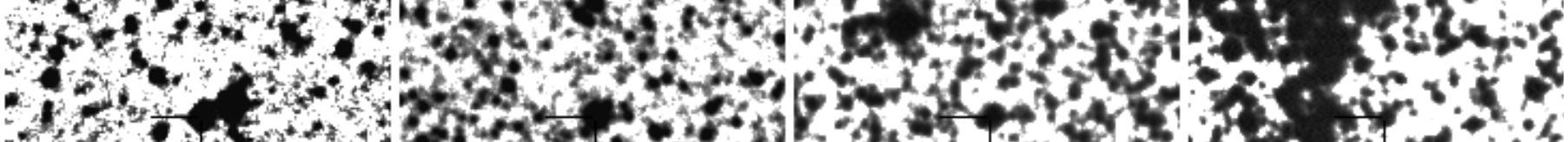

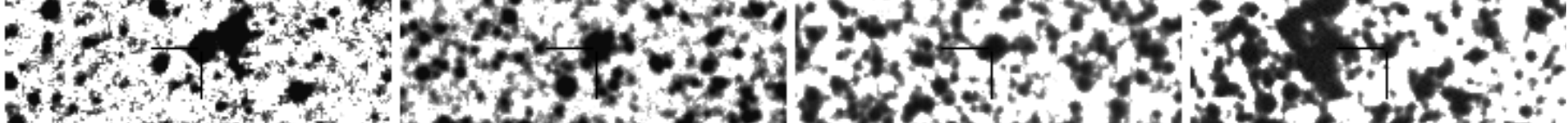

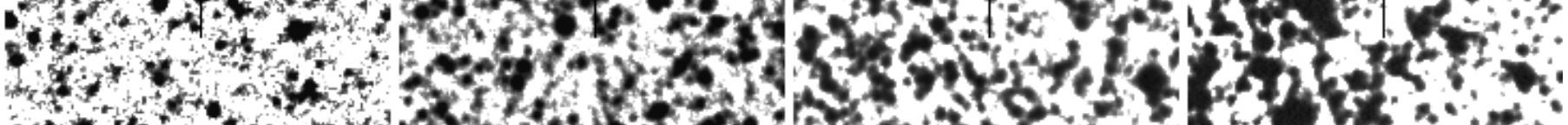

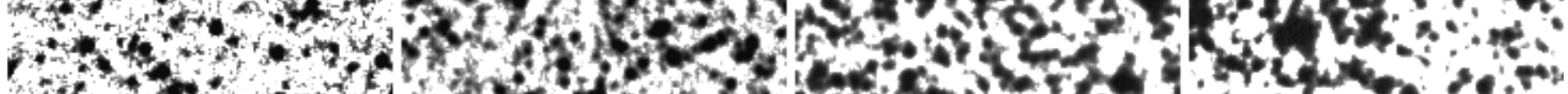

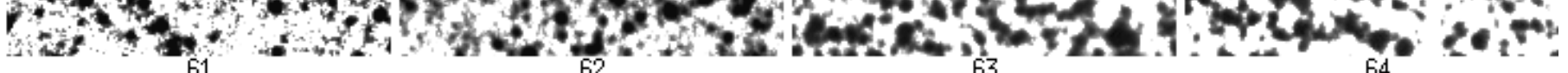


$\mathrm{N}$

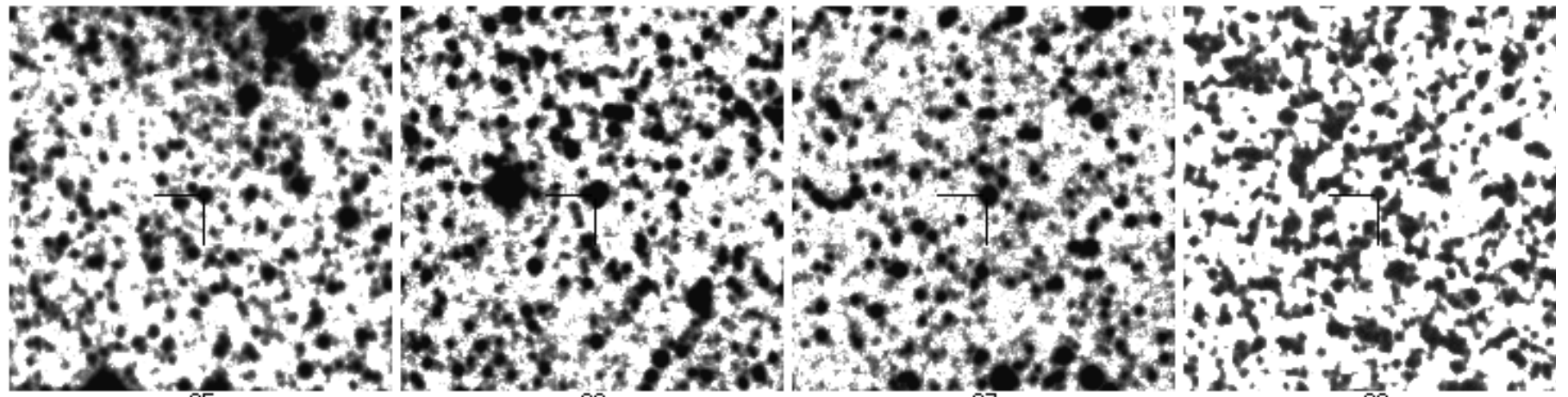

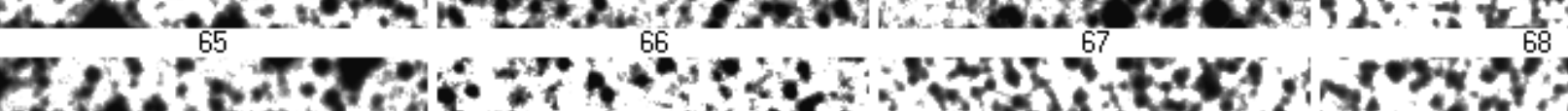

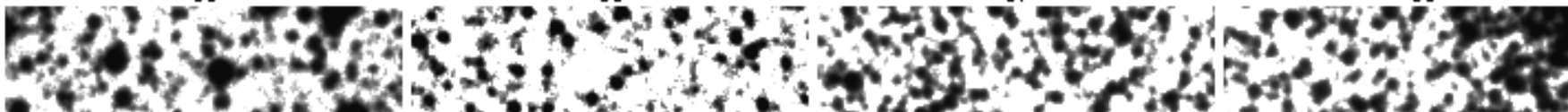

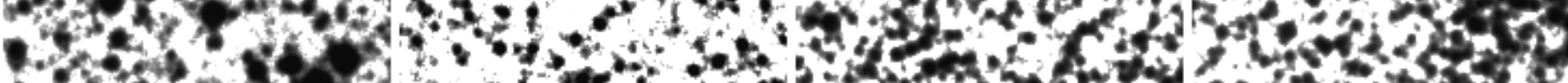

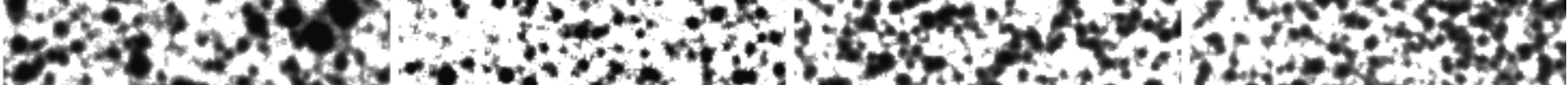

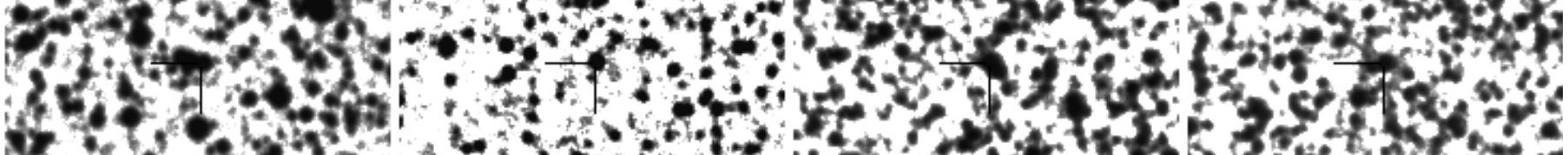

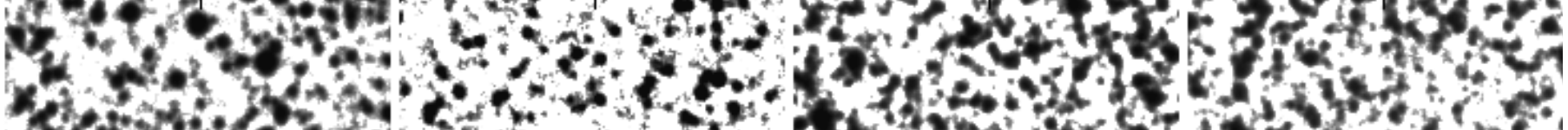

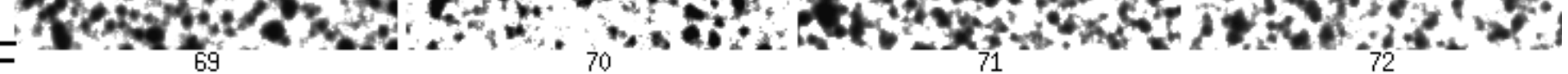

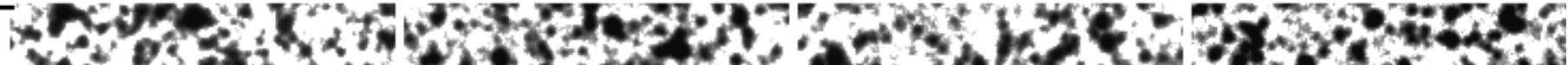

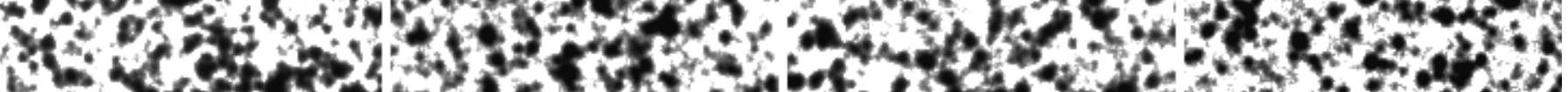

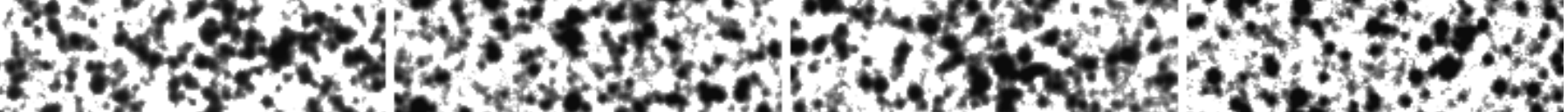

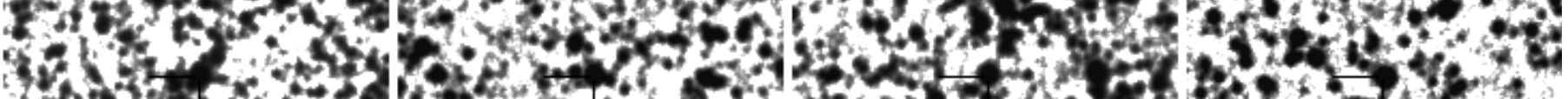

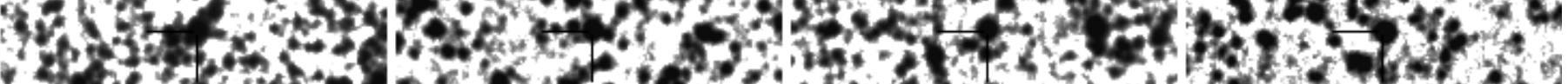

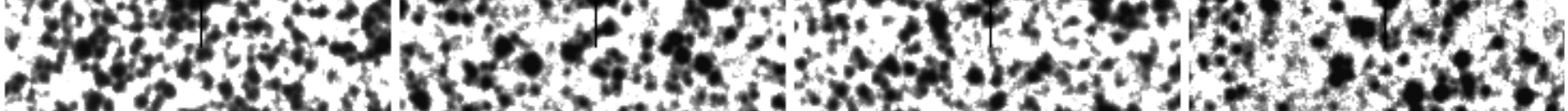

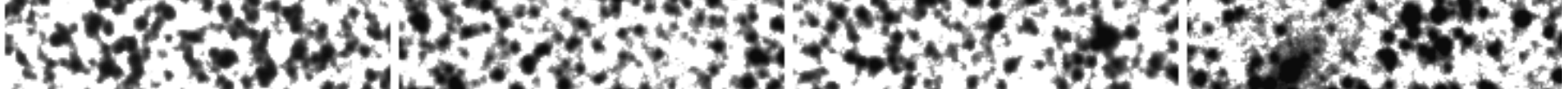

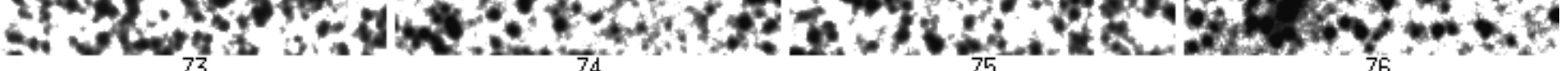

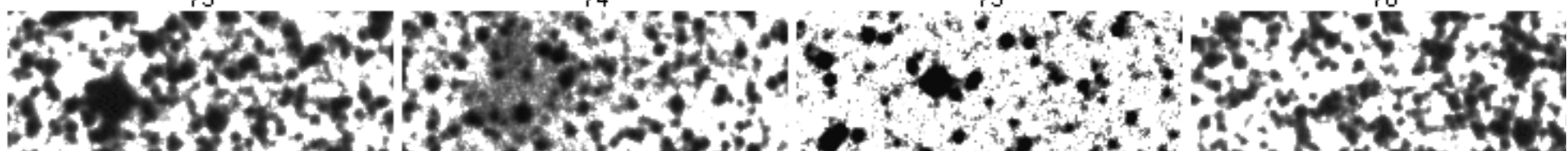

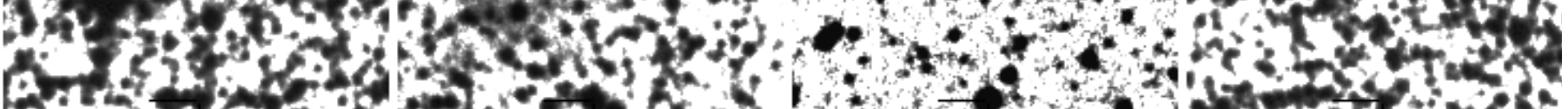

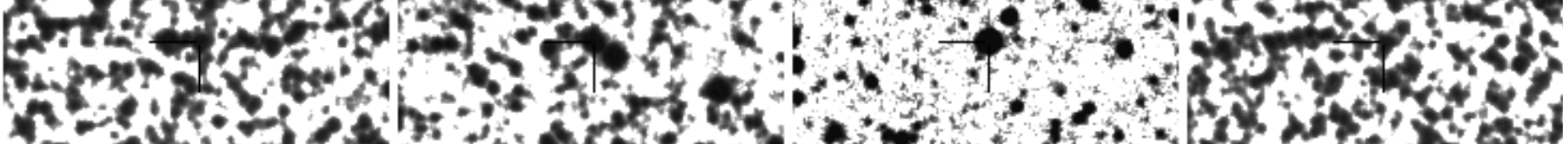

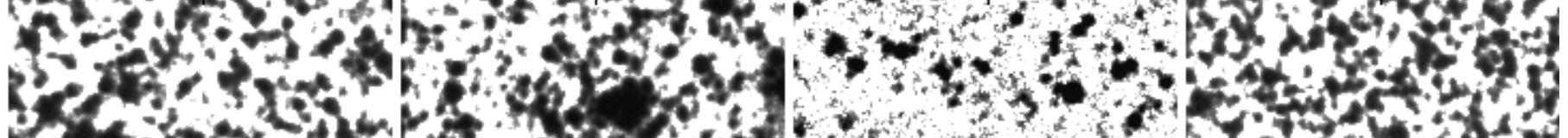

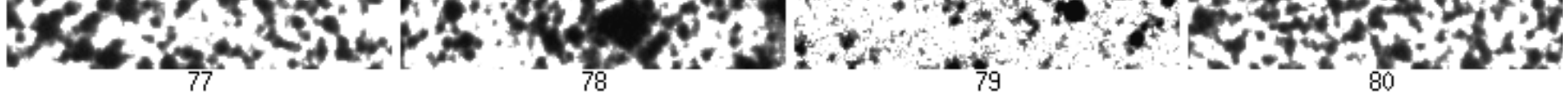


N

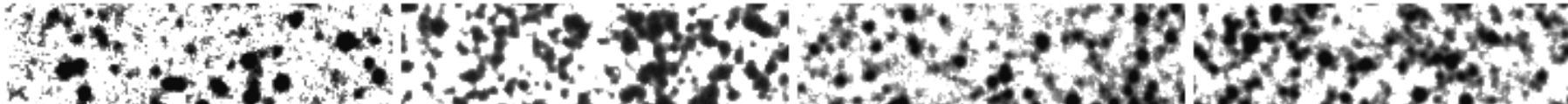

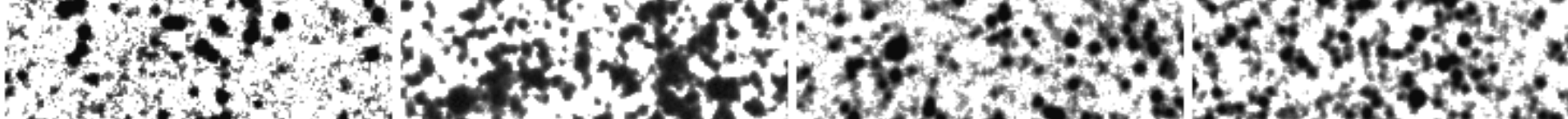

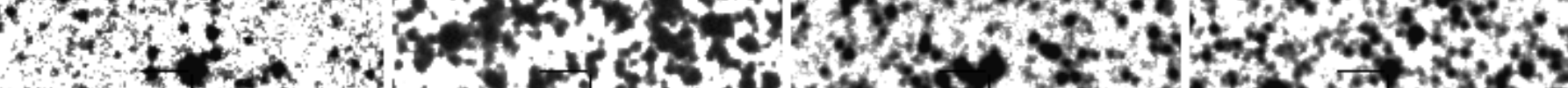

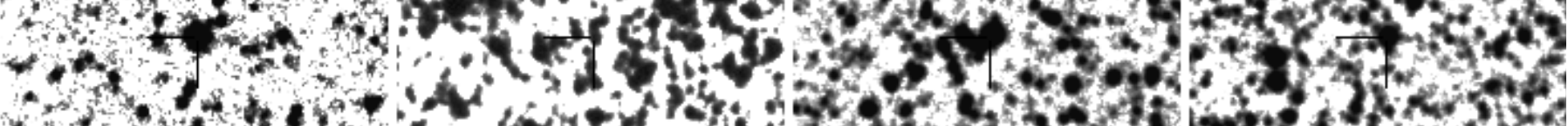

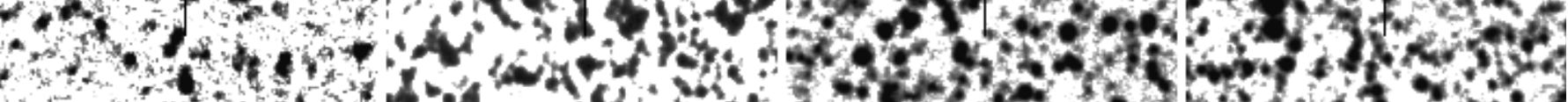

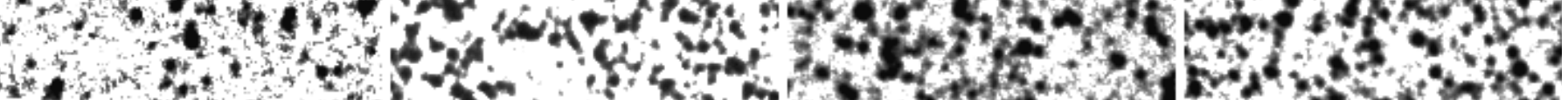

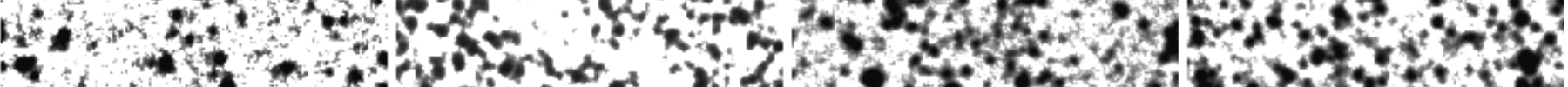

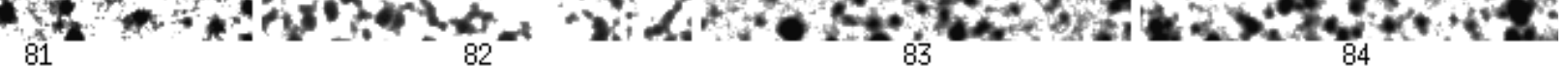

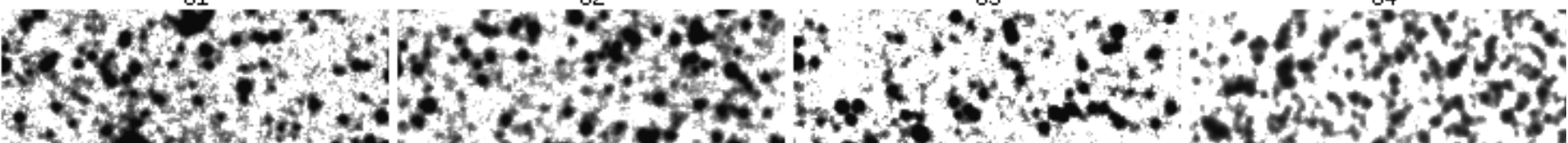

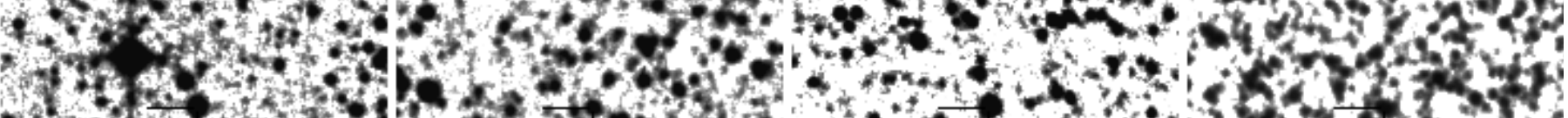

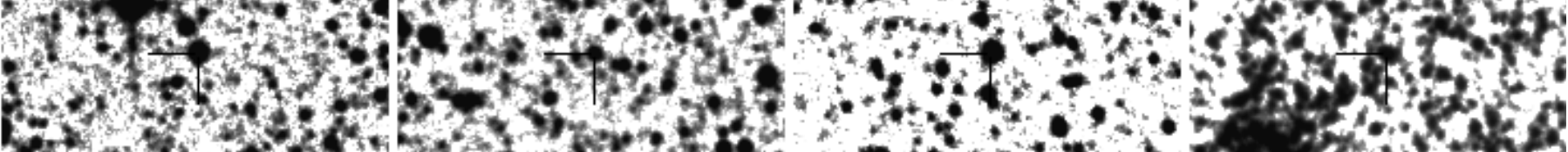

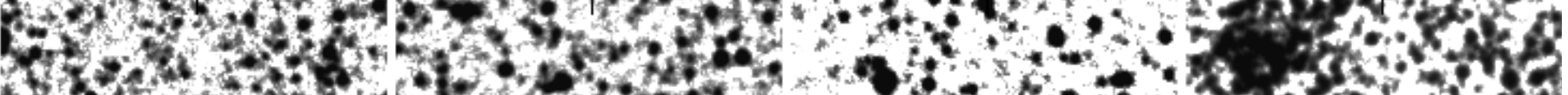

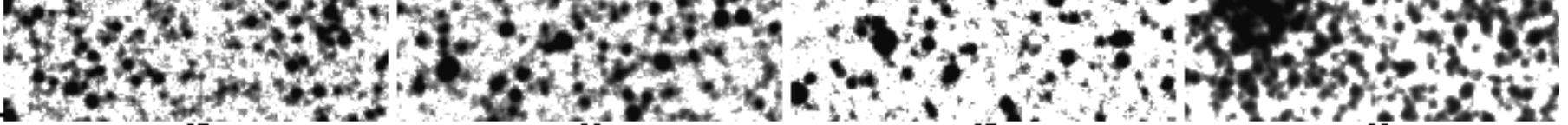

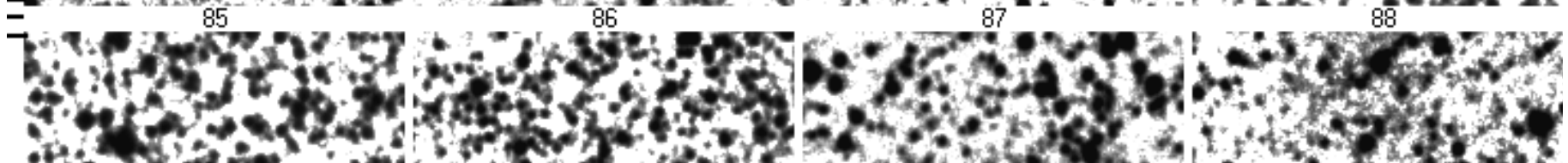

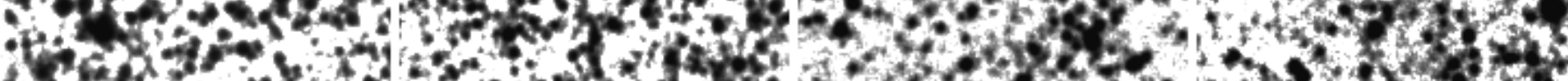

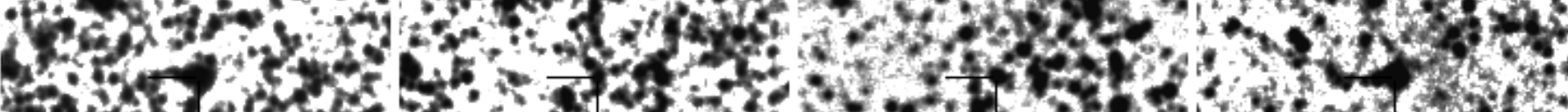

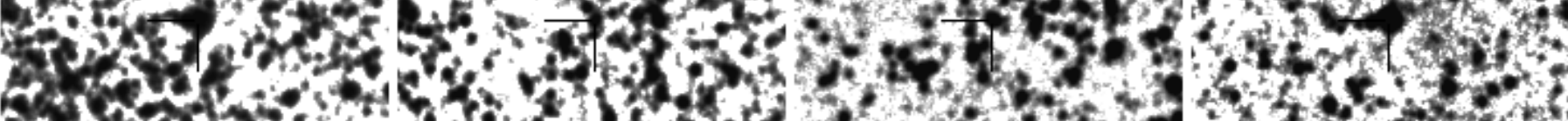

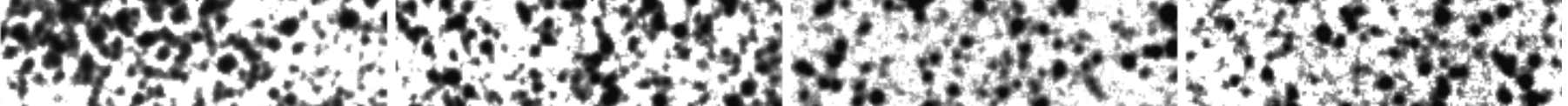

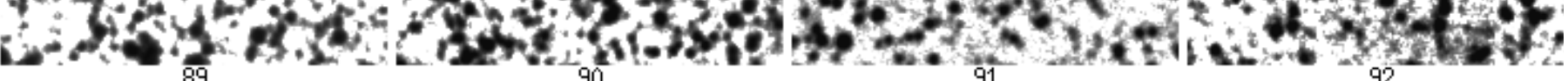
460

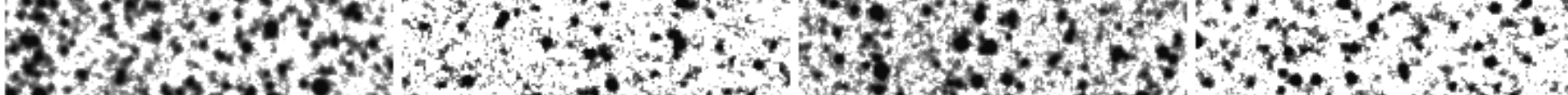

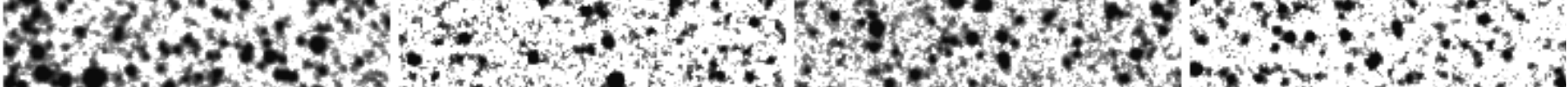

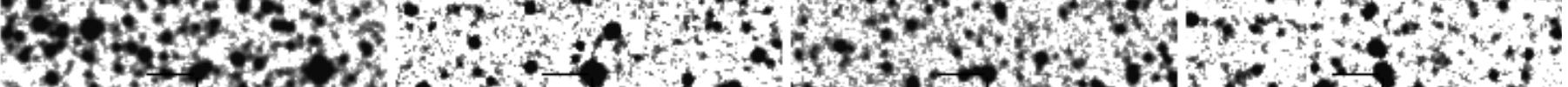

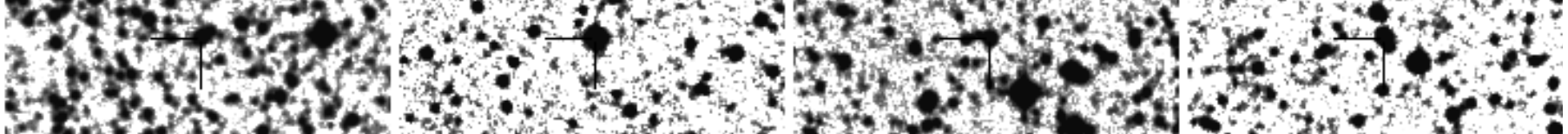

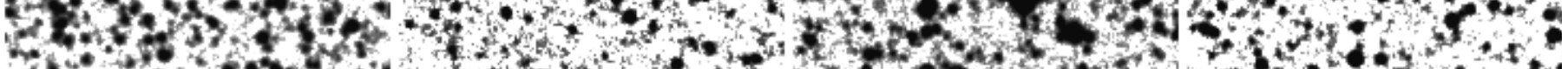

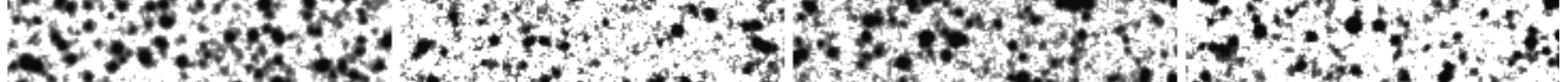

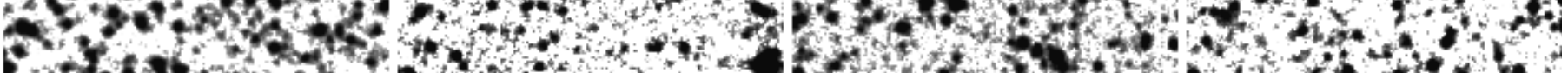

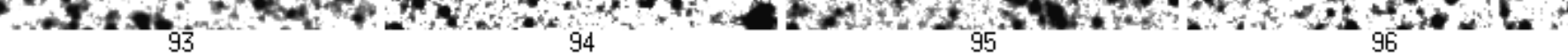


$\mathbf{N}$

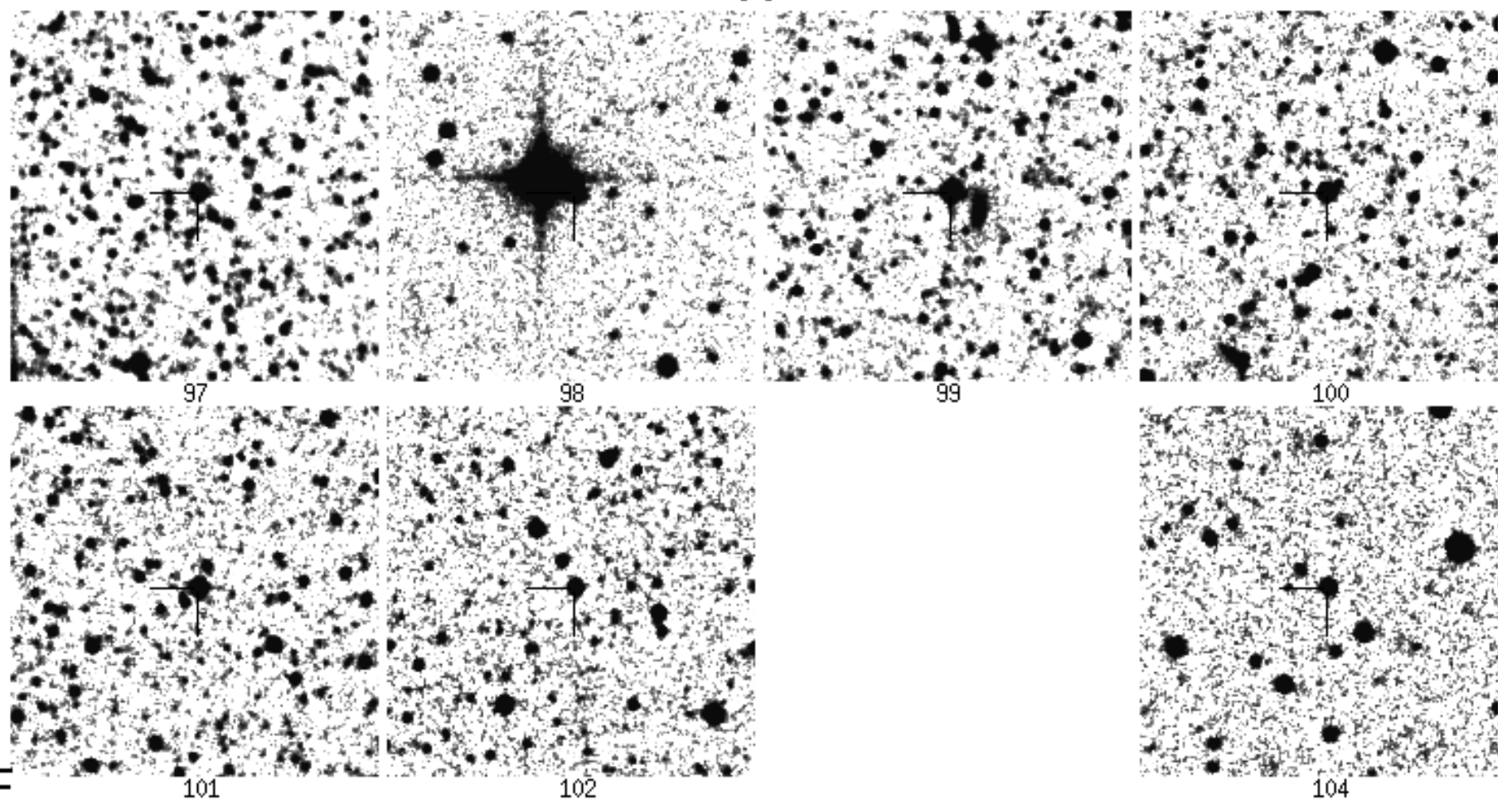

Fot.

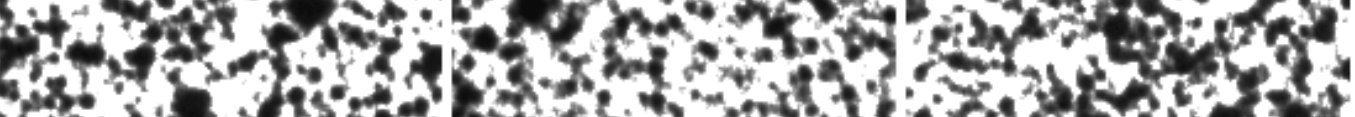

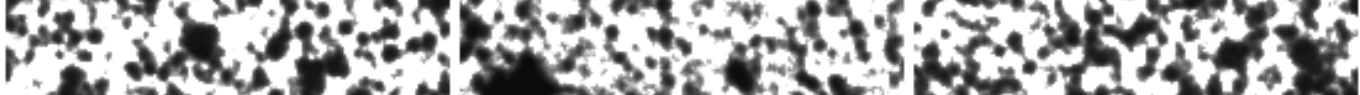

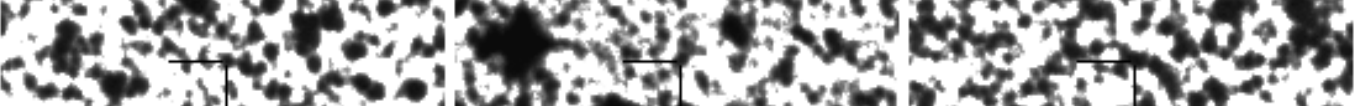

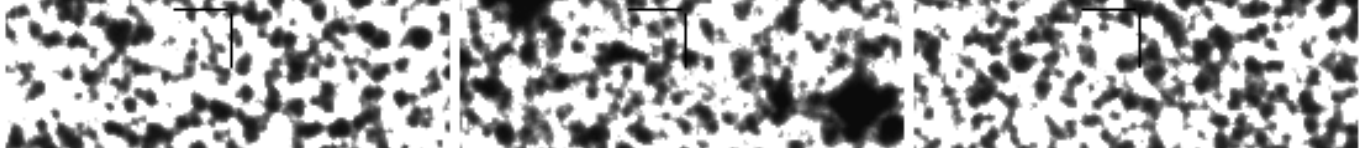

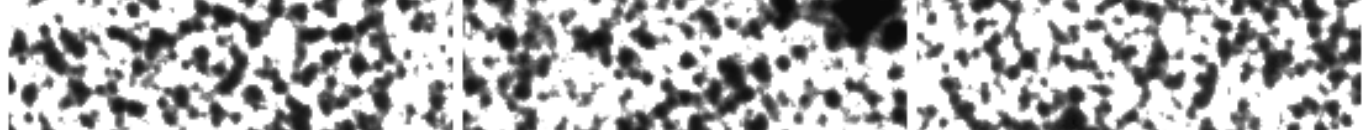

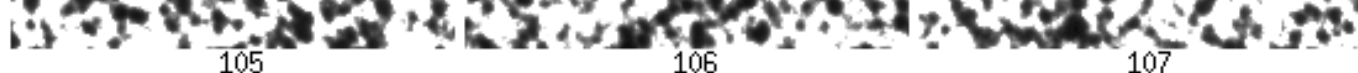

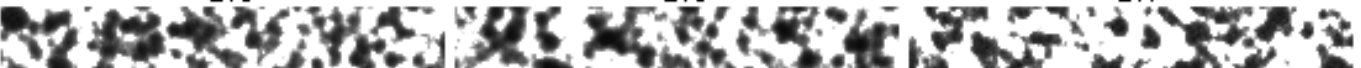

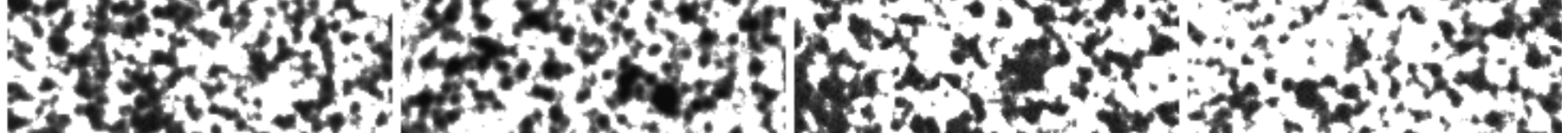
-

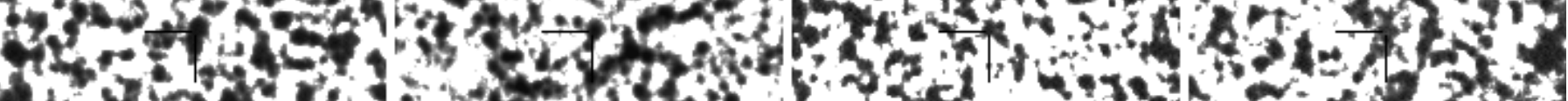

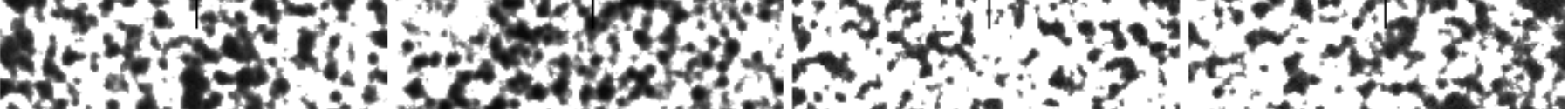

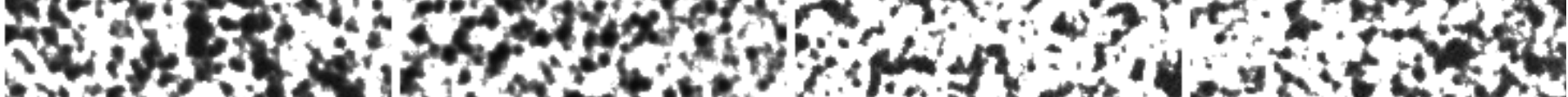

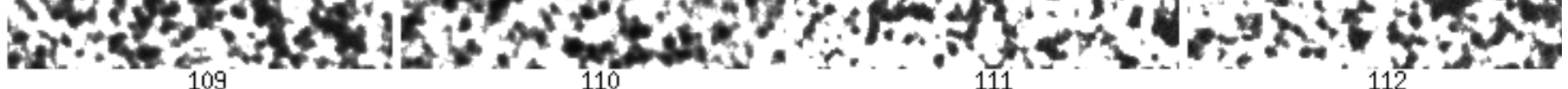

Fig. 13. Finding charts Sanduleak et al. 
$\mathrm{N}$

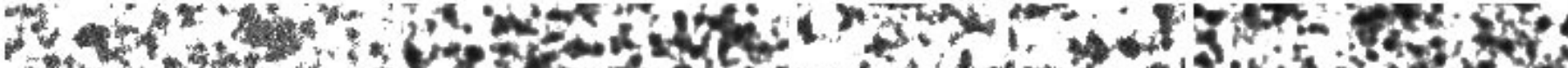

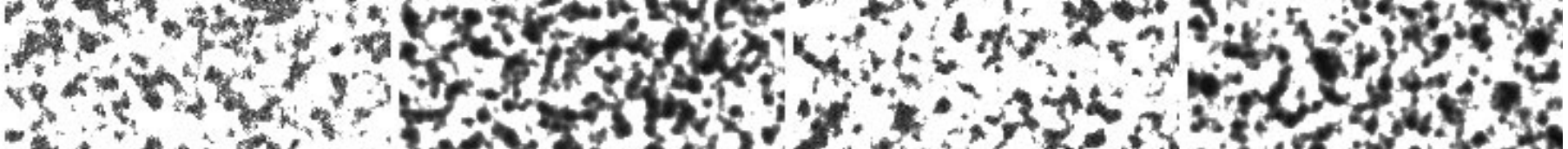

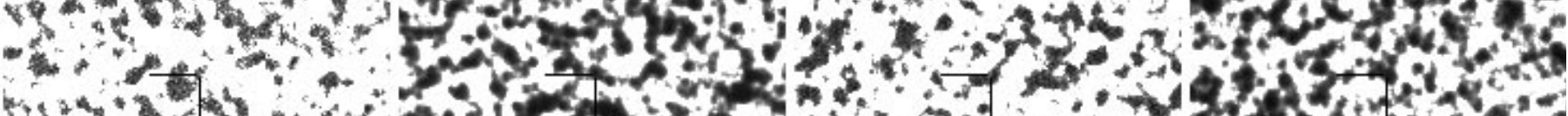

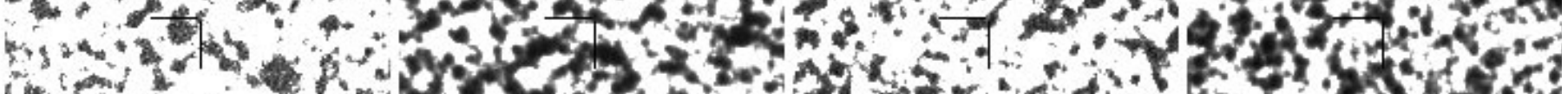

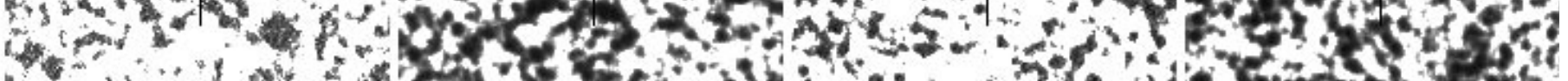
* a

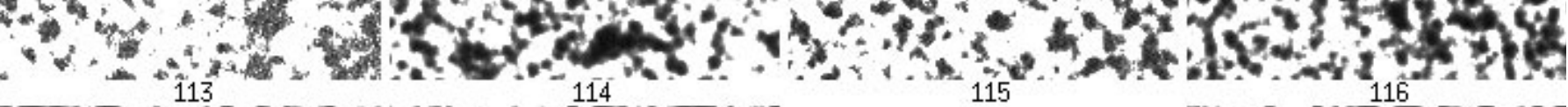

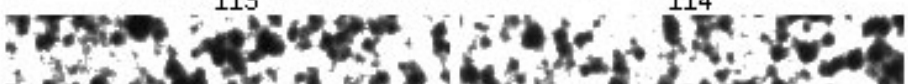

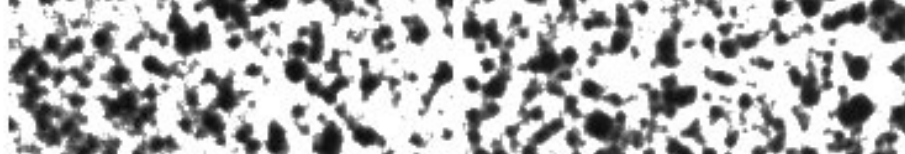

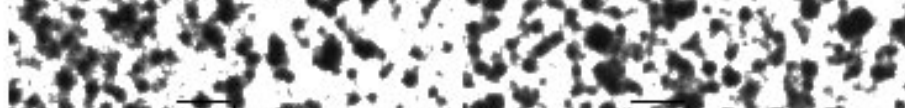

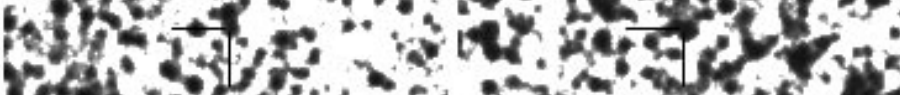

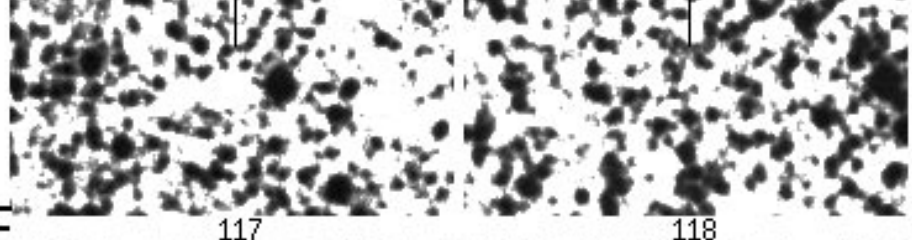

$=$

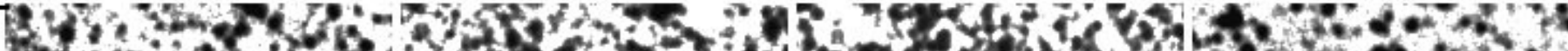

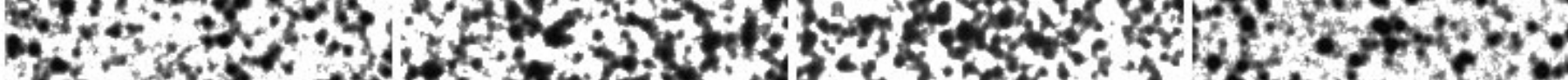

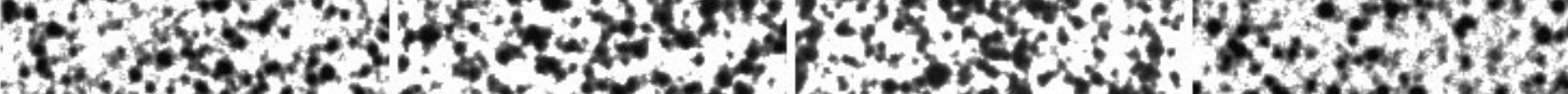

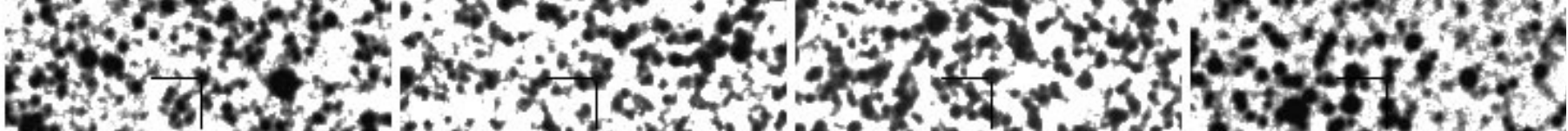

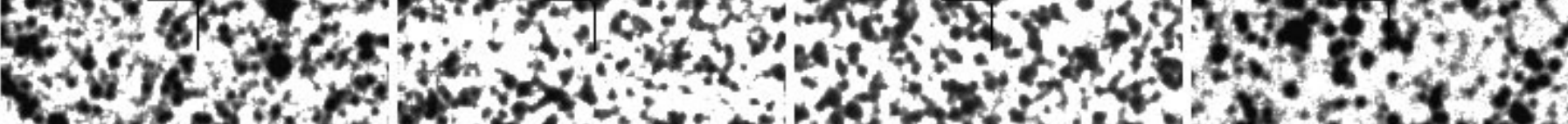

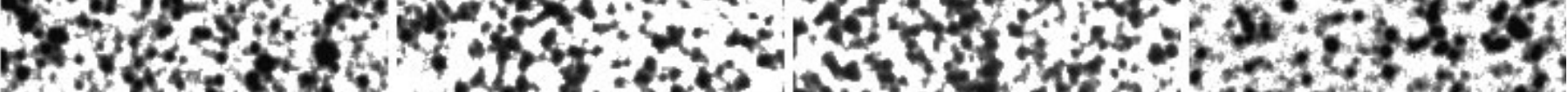

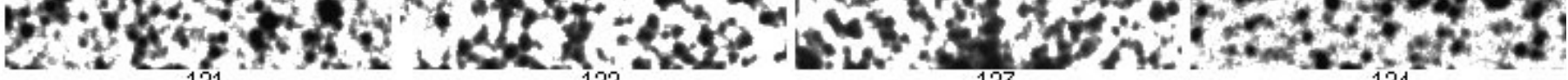

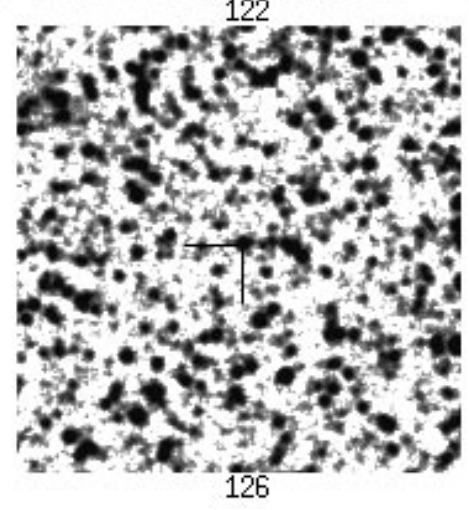

Fig. 14. Finding charts Sanduleak et al 
$\mathrm{N}$

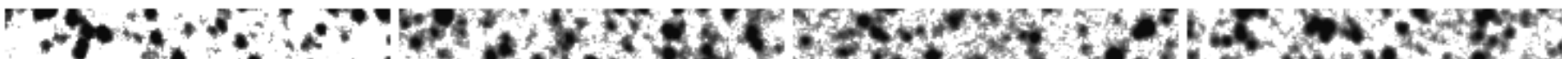

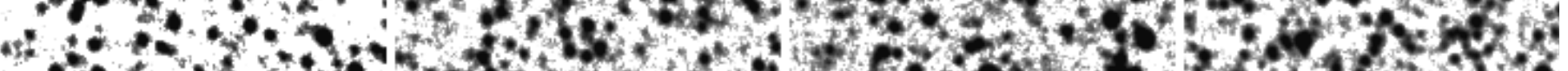

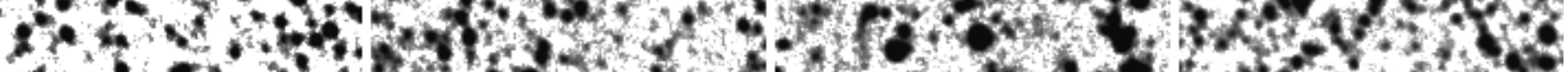

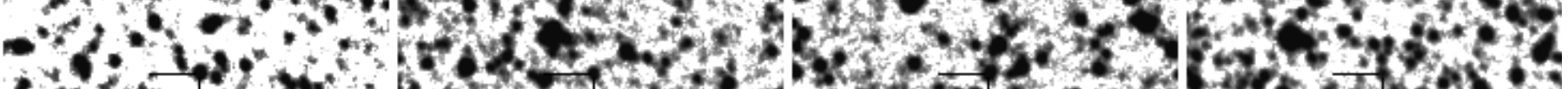

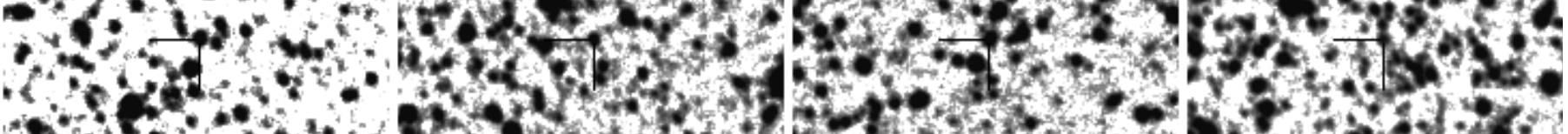

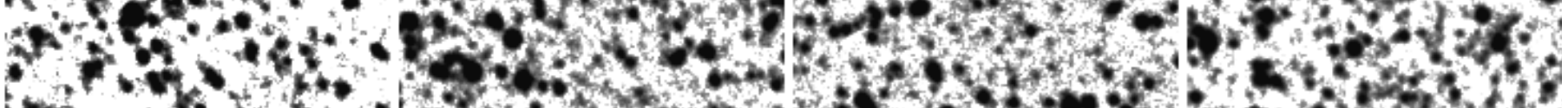

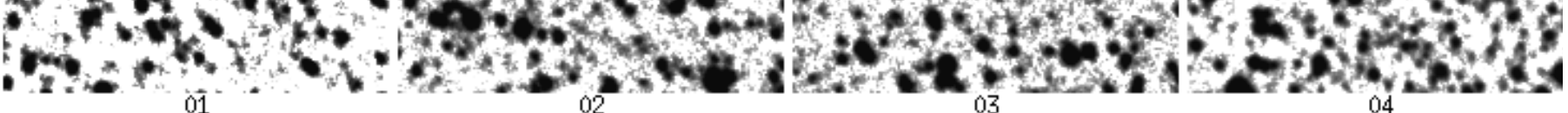

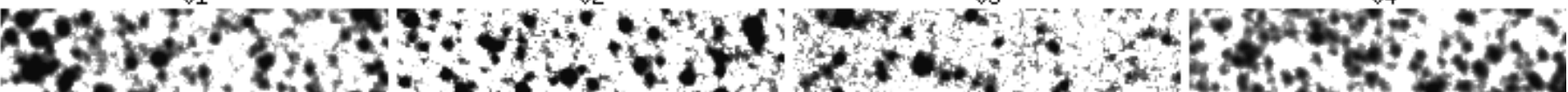

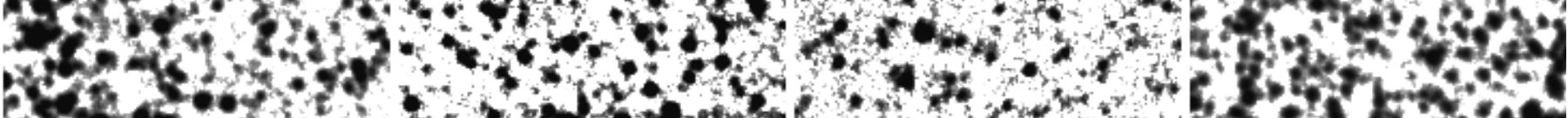
P.t

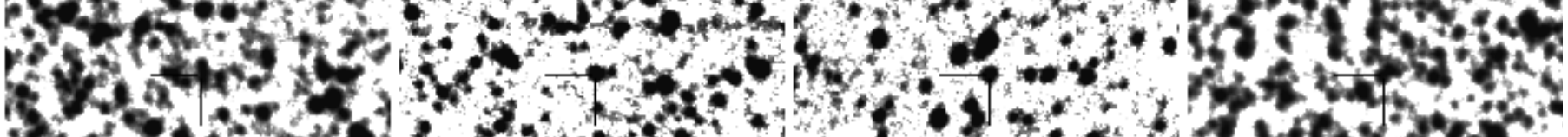

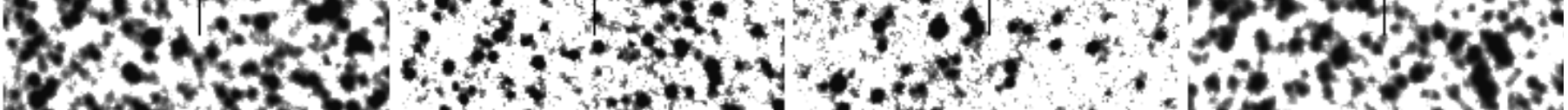

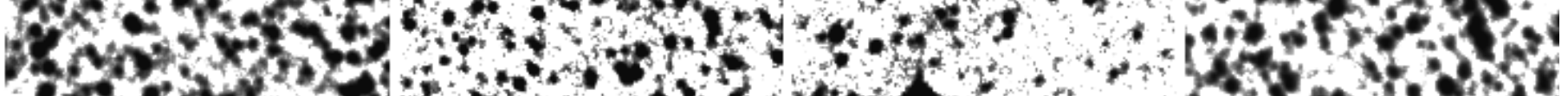

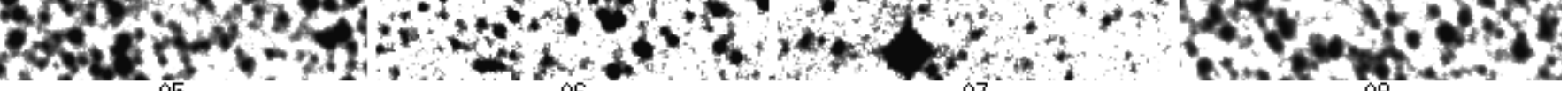

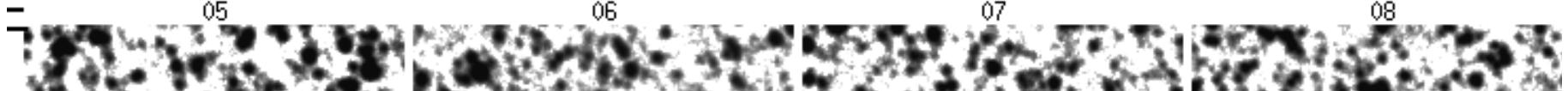

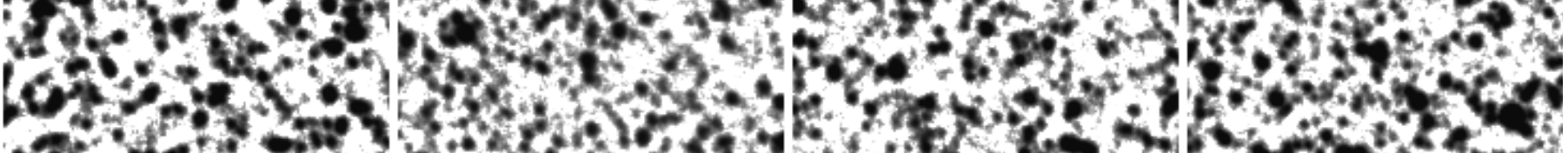
s.

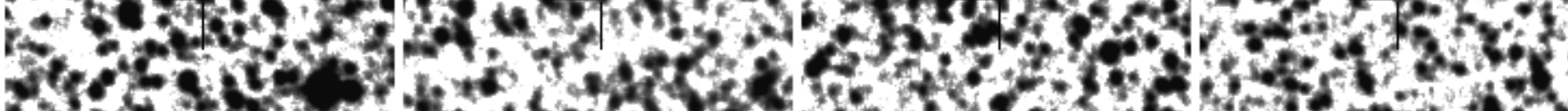

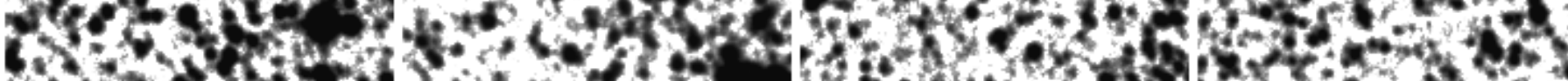

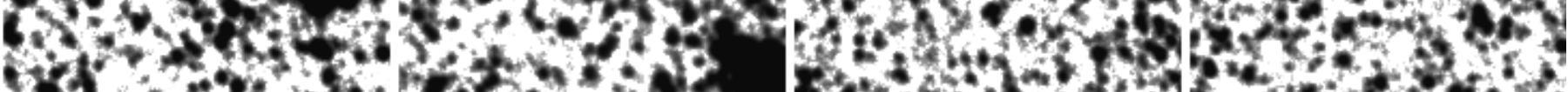

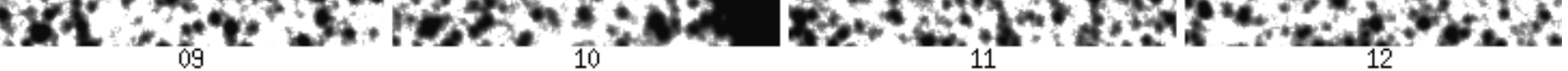

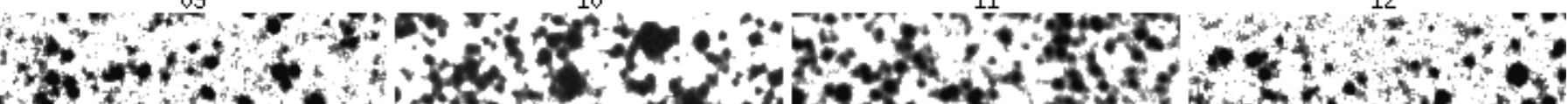

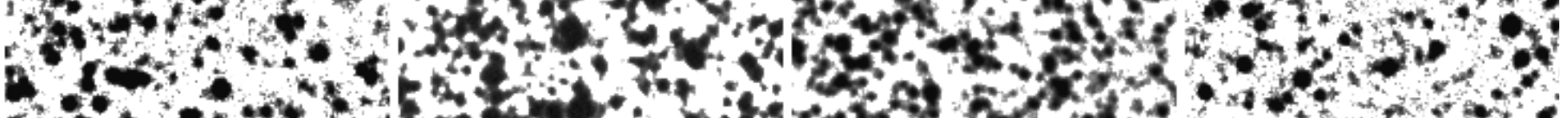

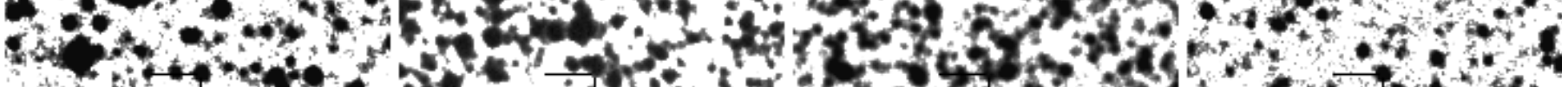

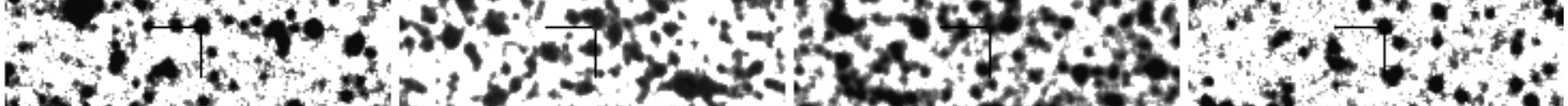

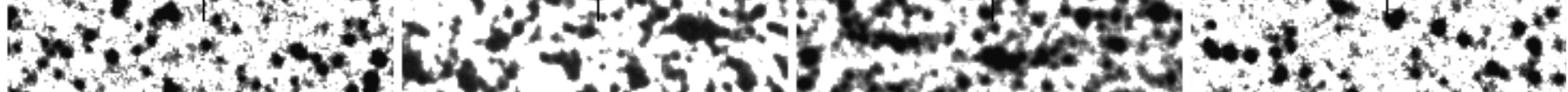

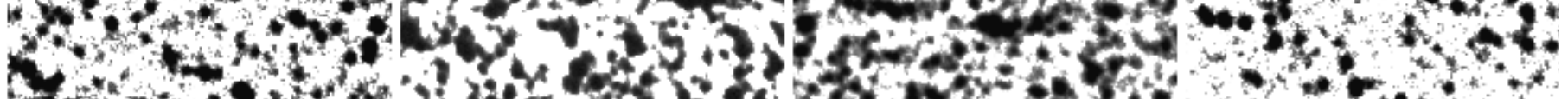

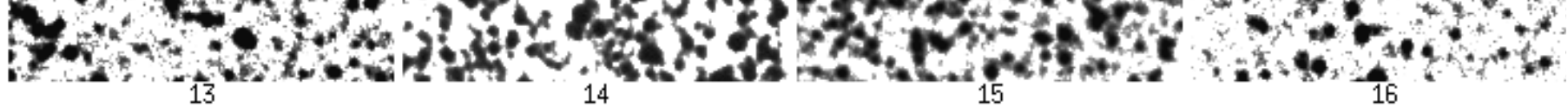


$\mathrm{N}$

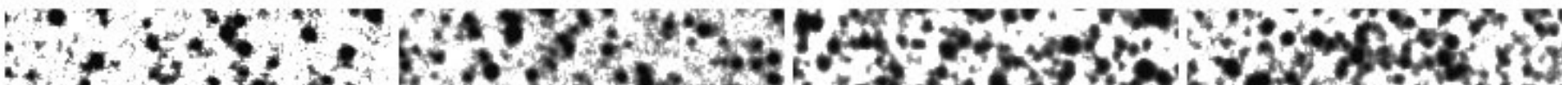

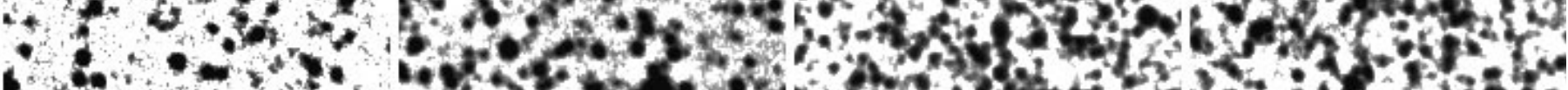

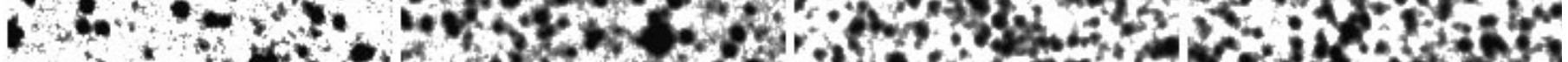

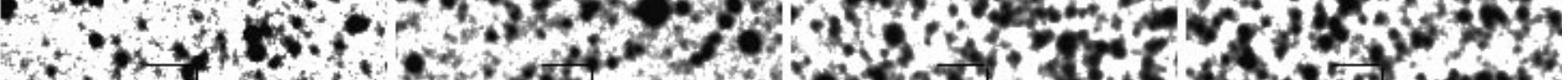

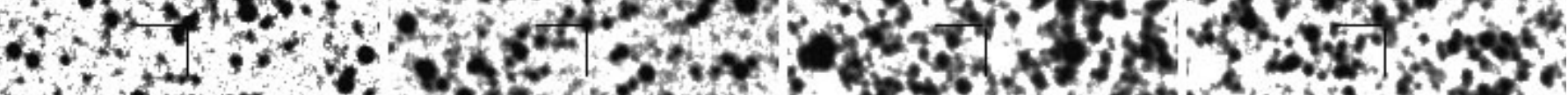

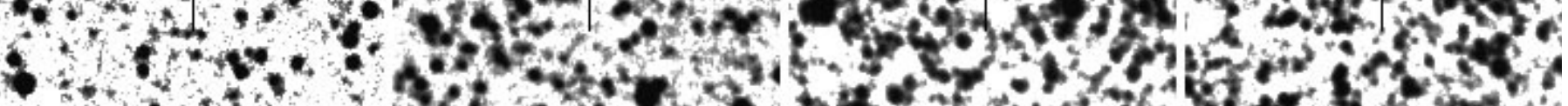

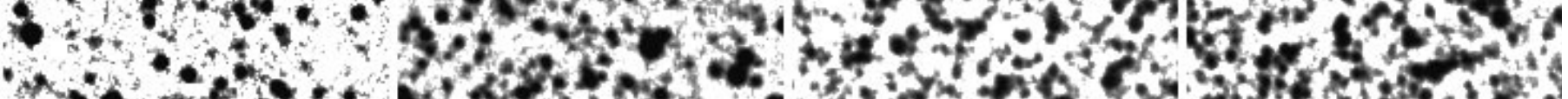

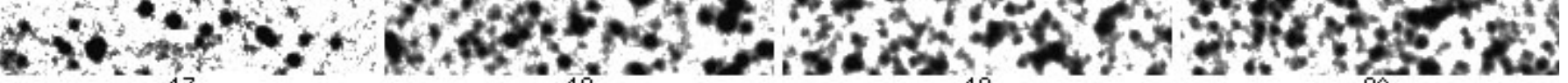

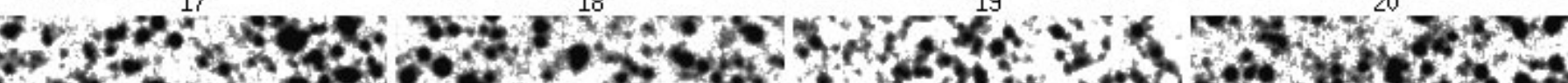
7.5.

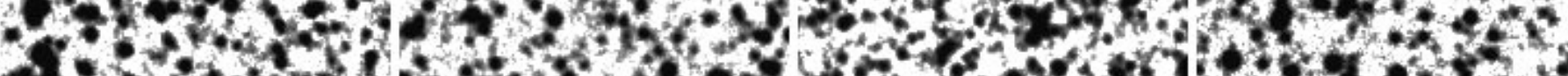

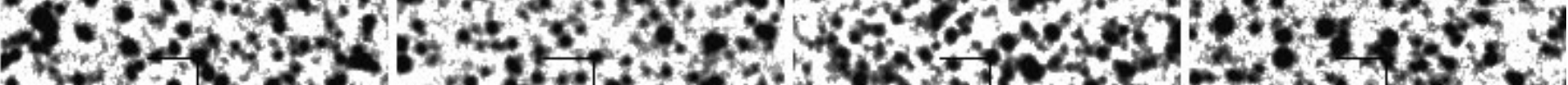

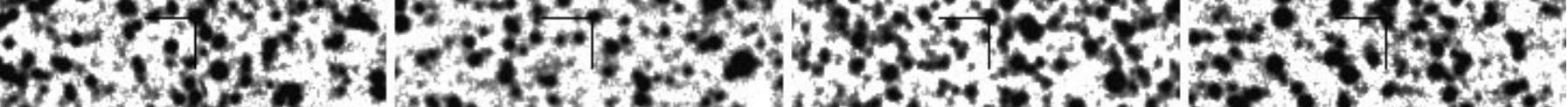

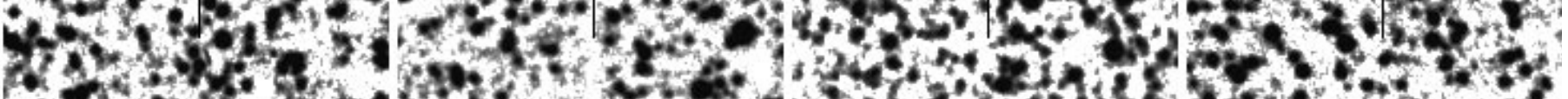

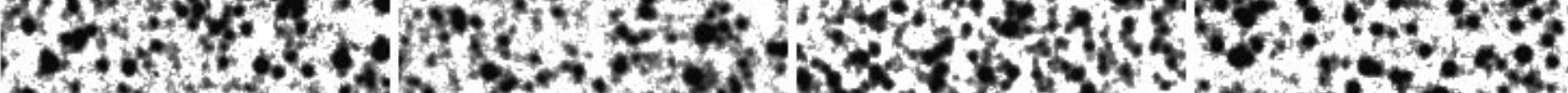
E.

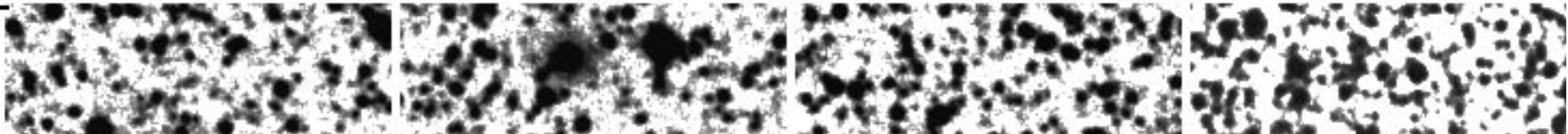

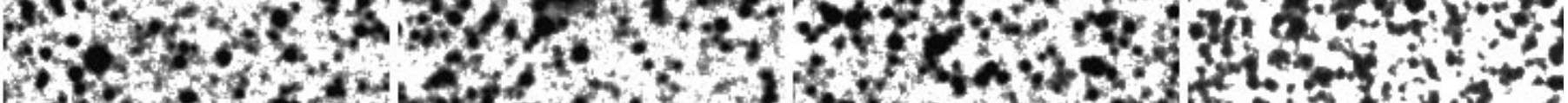

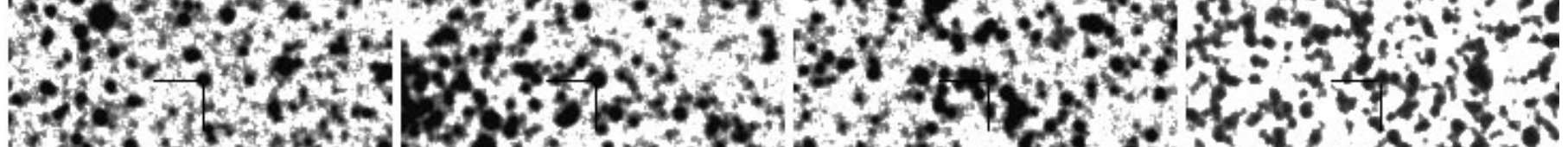

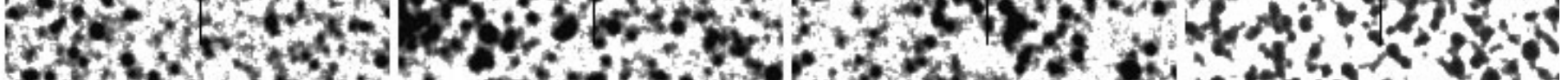

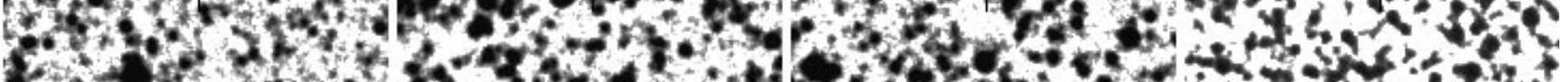

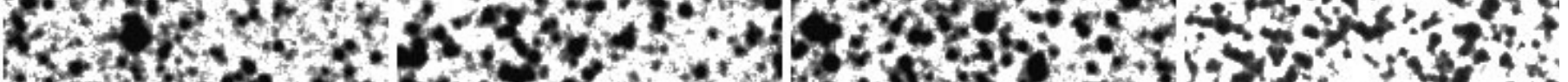

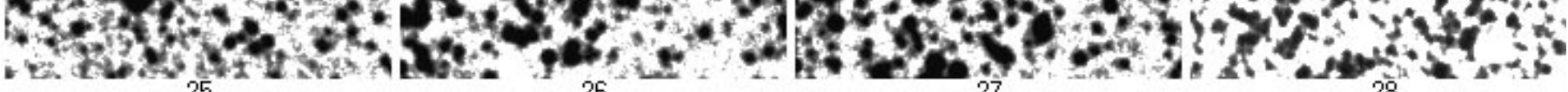

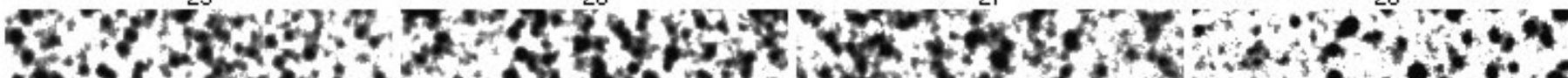

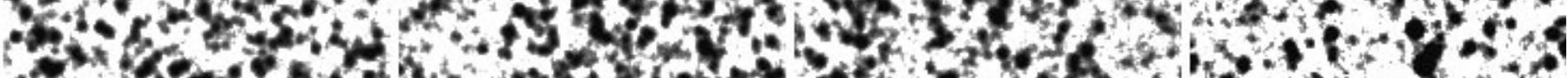

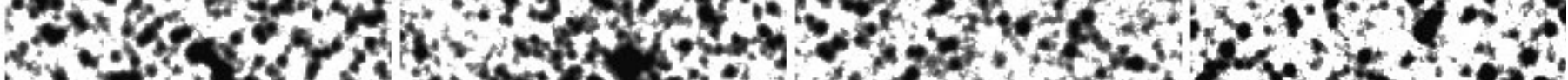

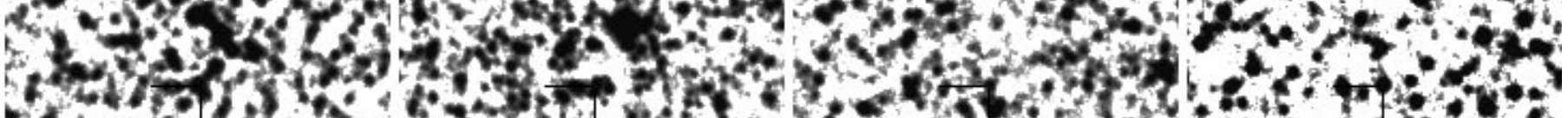

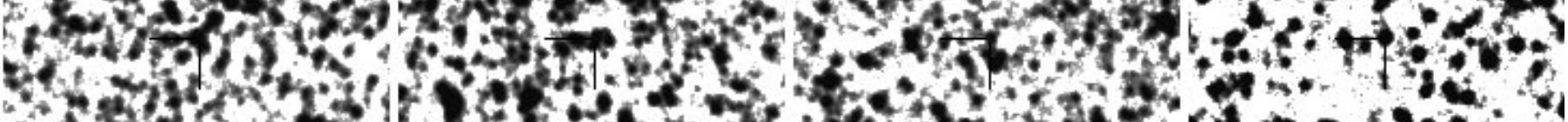

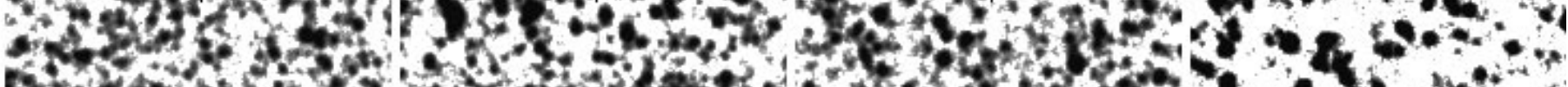

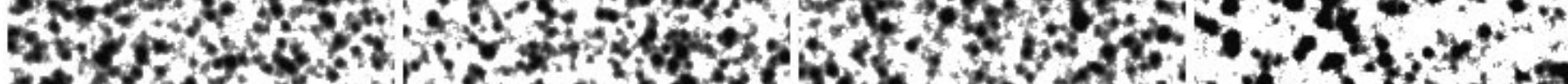

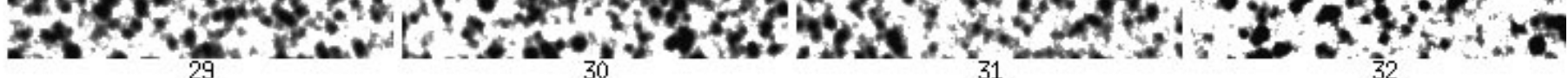


$\mathrm{N}$

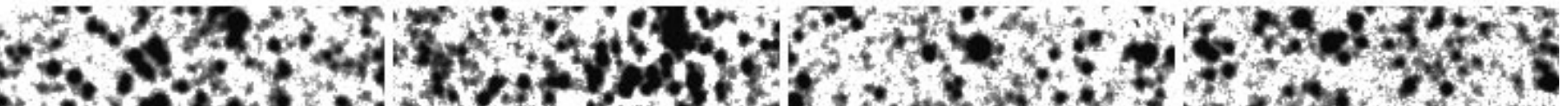

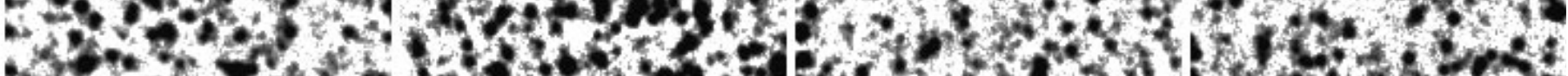

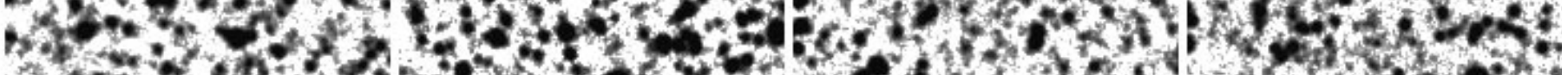

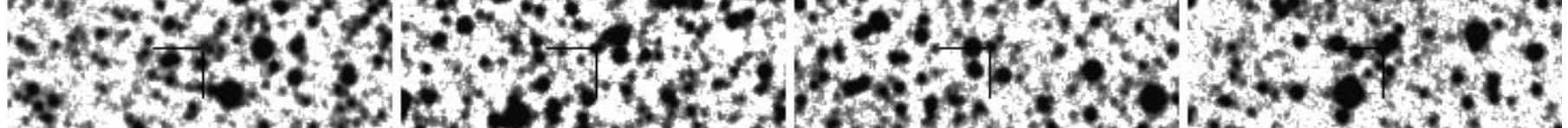

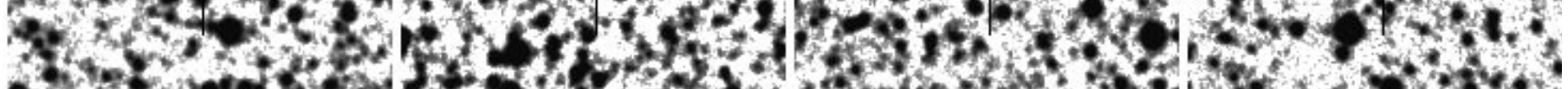

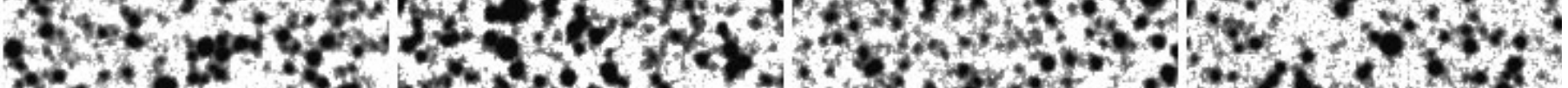

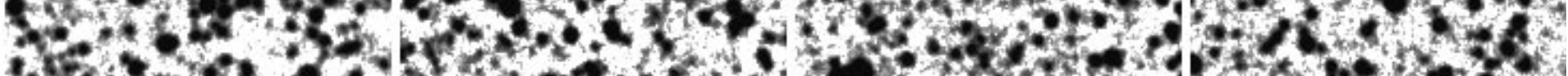

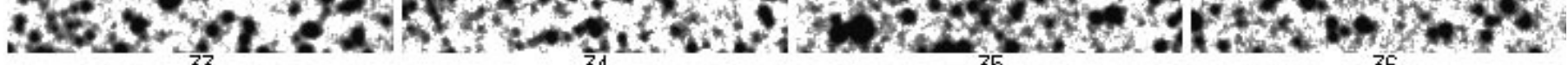

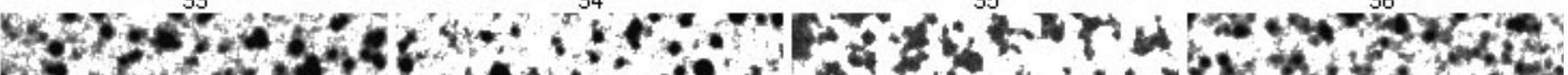

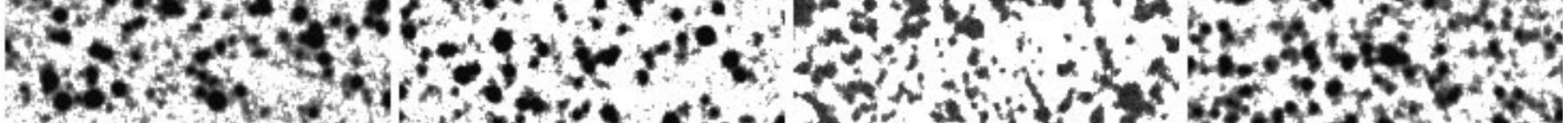

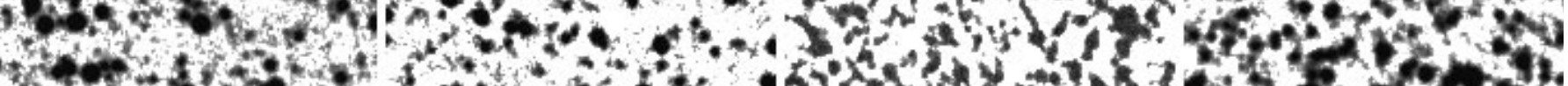

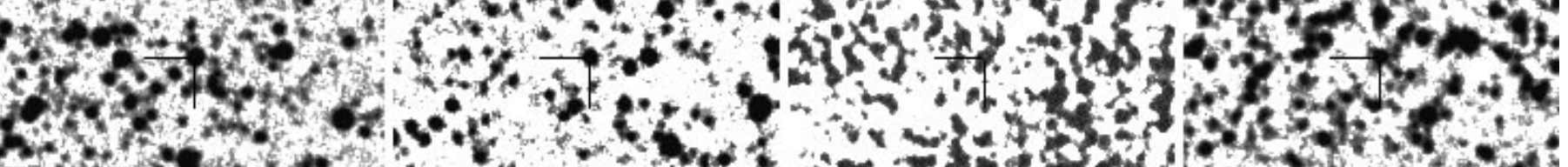
2.5.

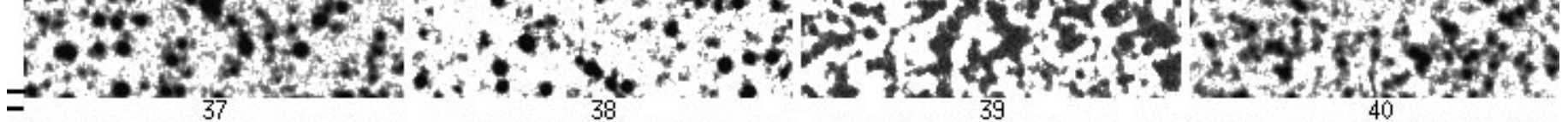

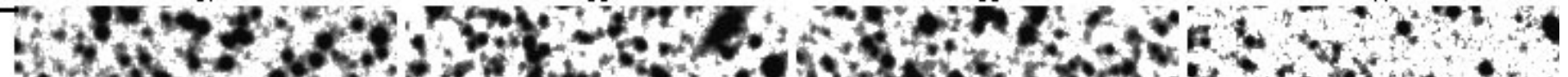

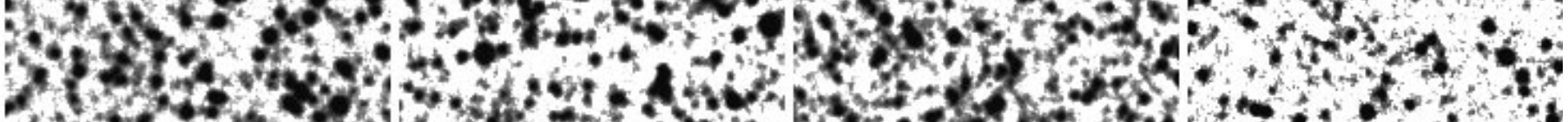

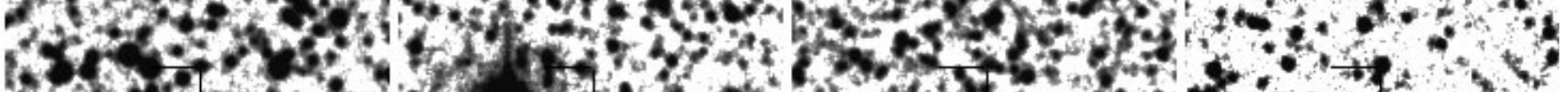

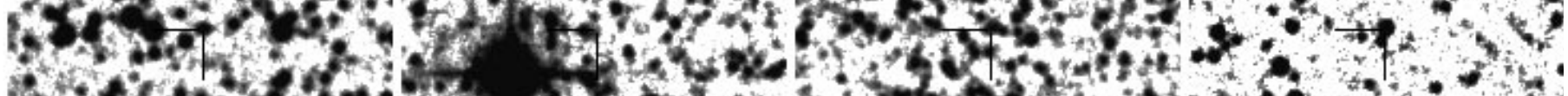

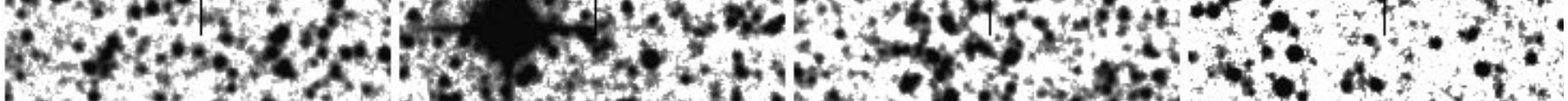

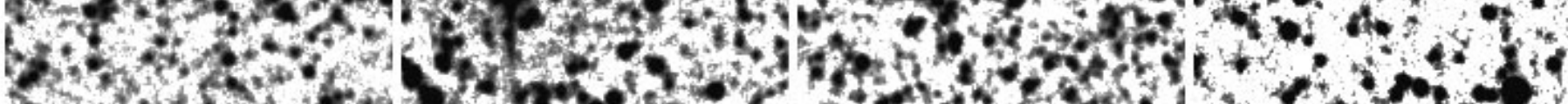

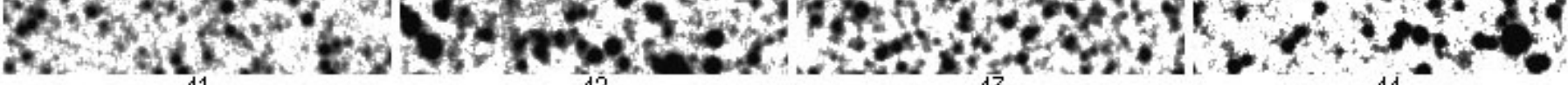

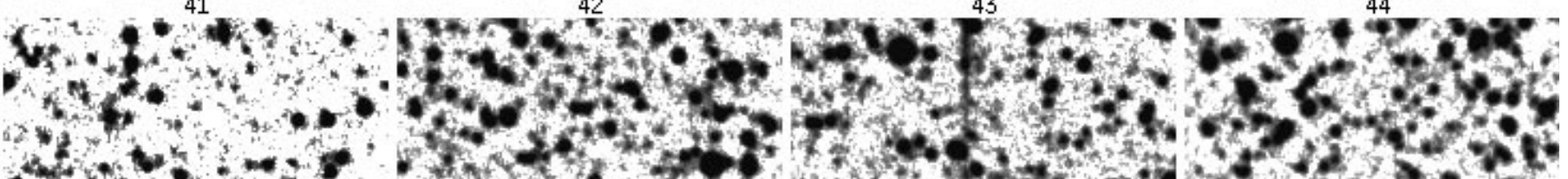

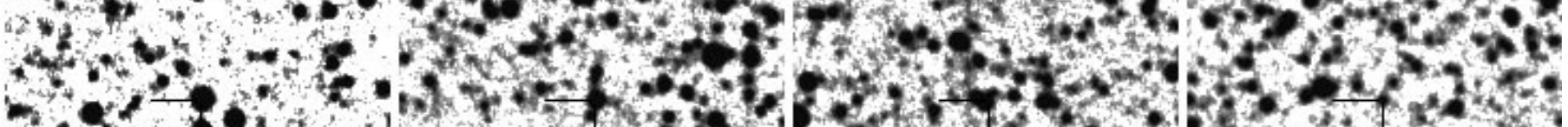

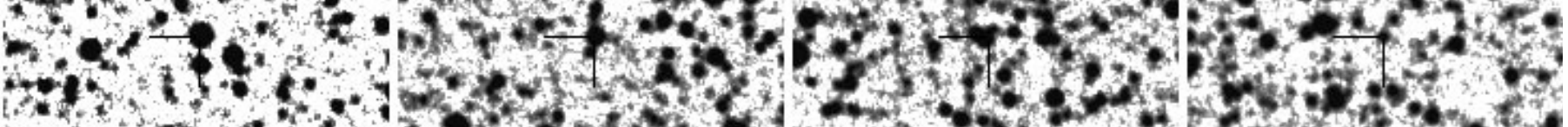

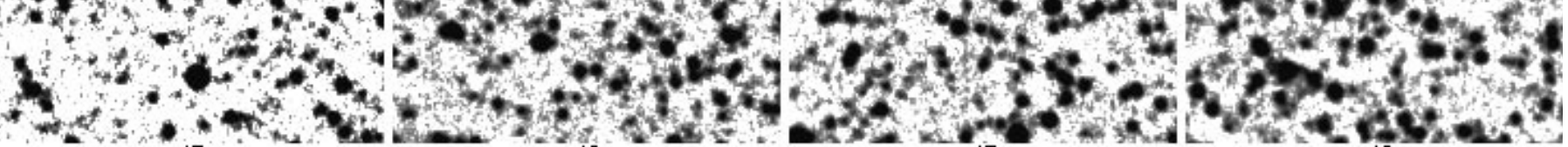

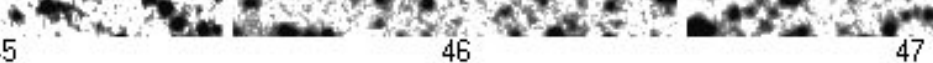


$\mathbf{N}$

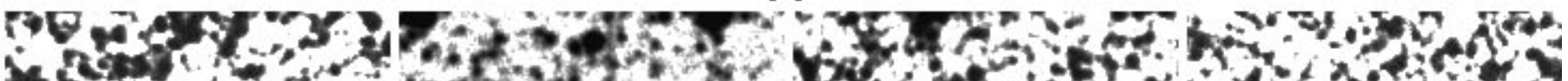

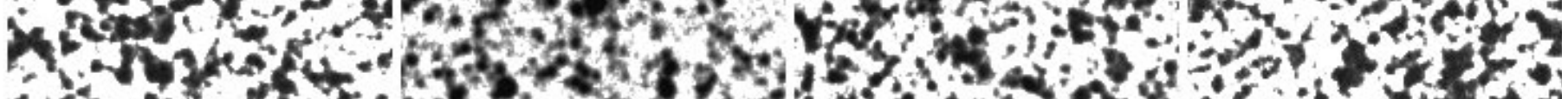

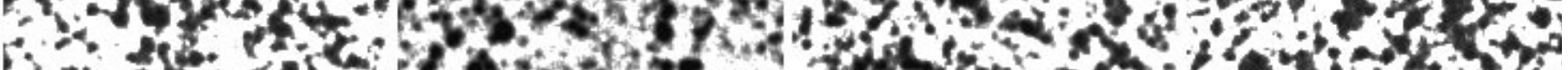

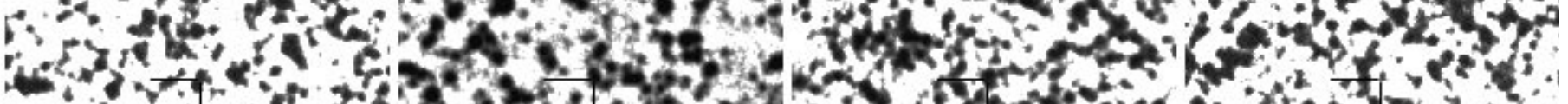
this

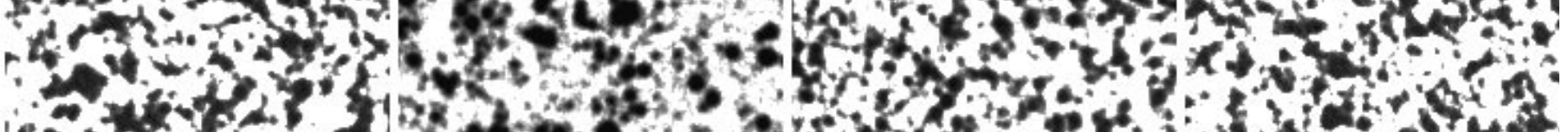

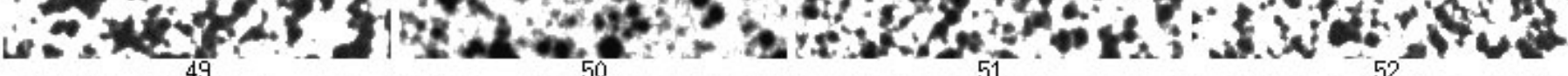

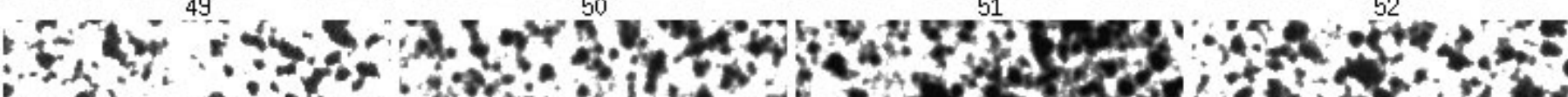

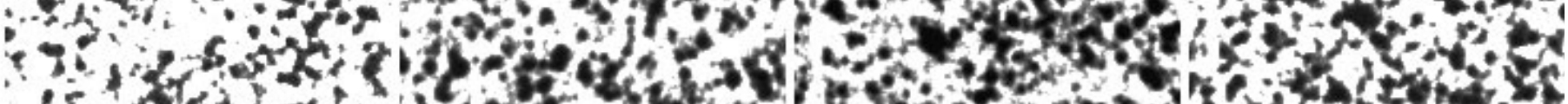

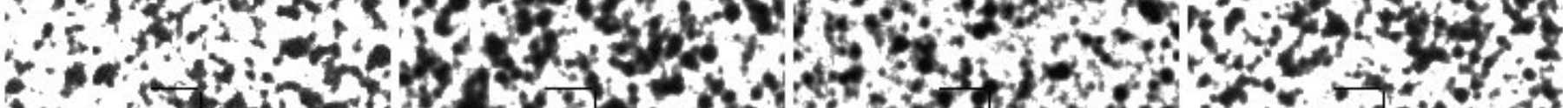

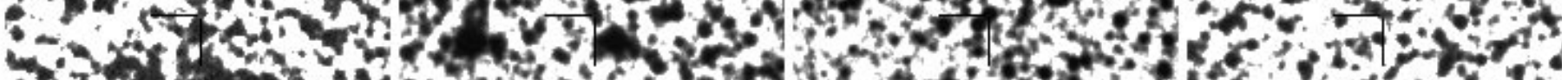

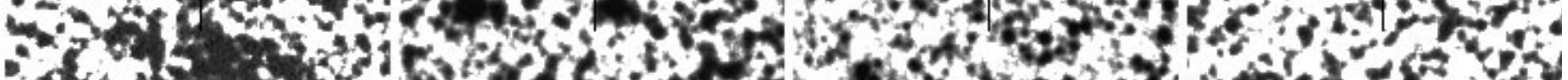
cot

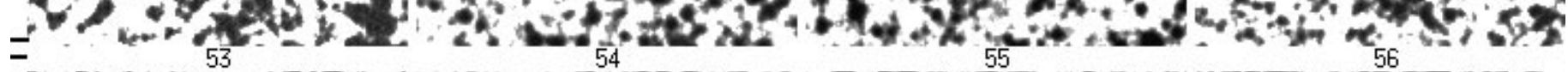

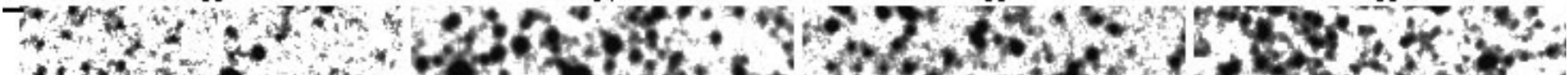

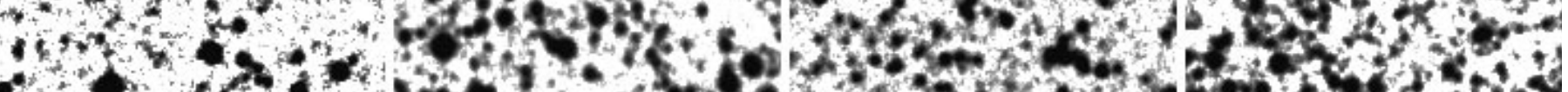

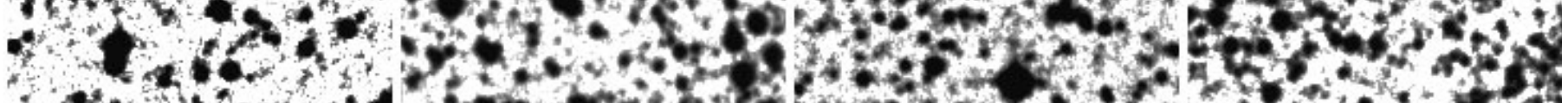

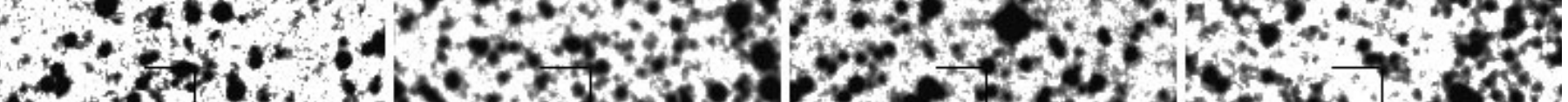

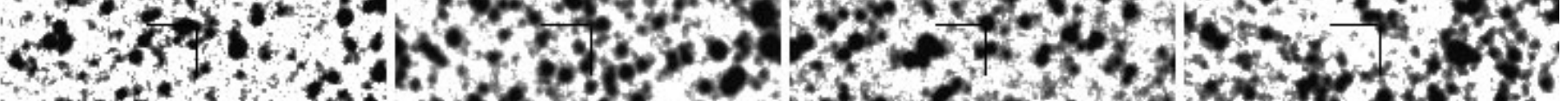

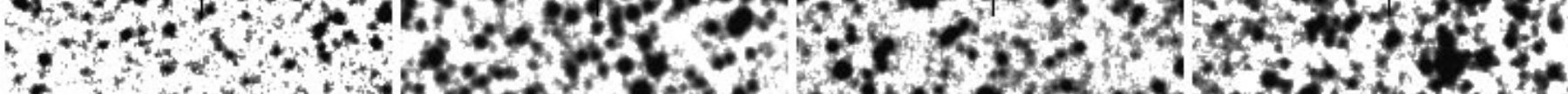

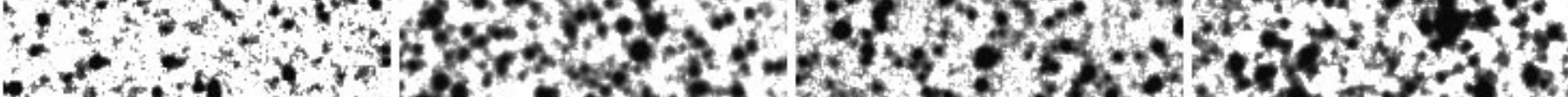

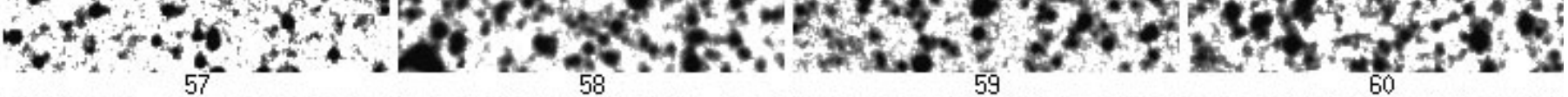

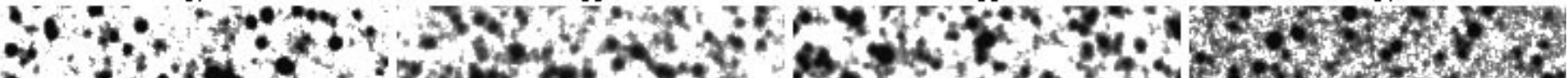

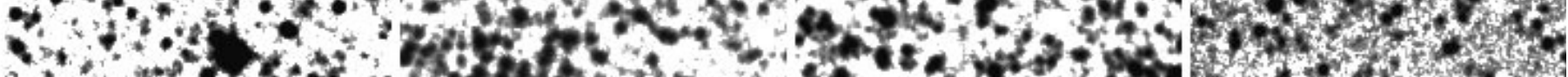

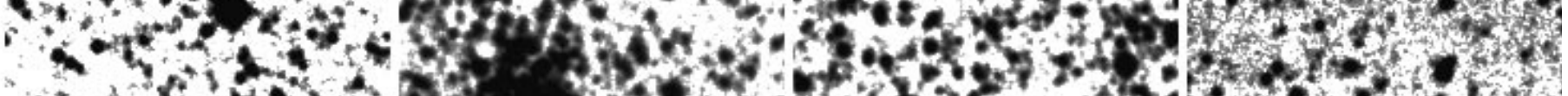

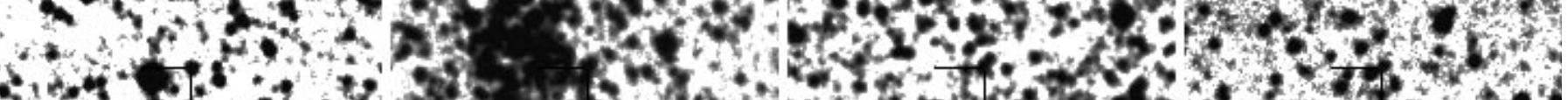

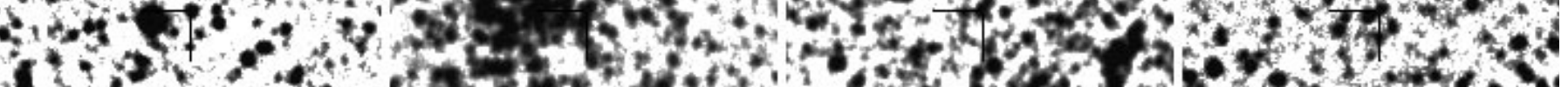

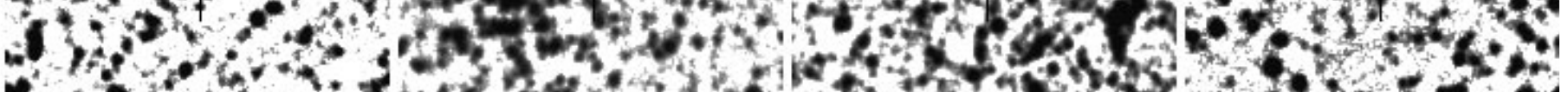

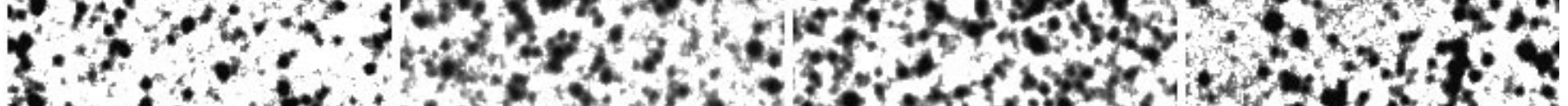

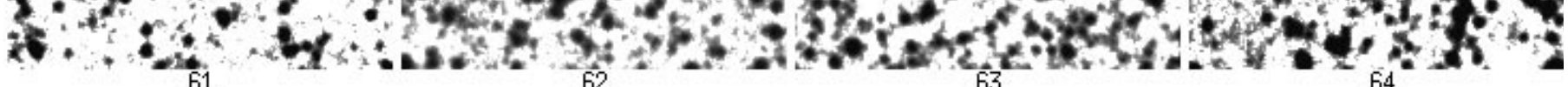




\section{$\mathbf{N}$}

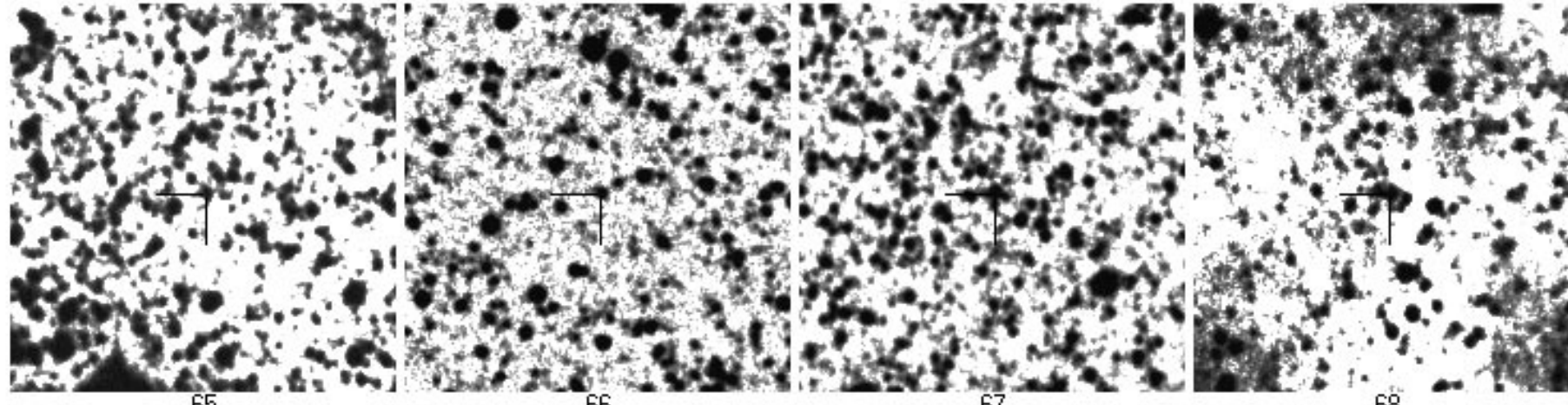

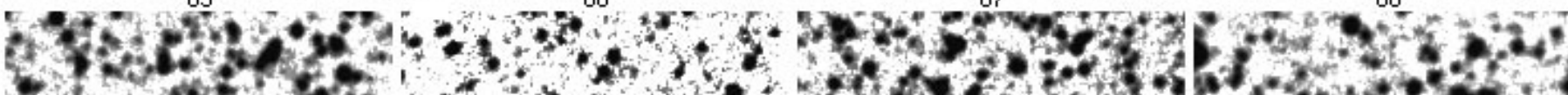

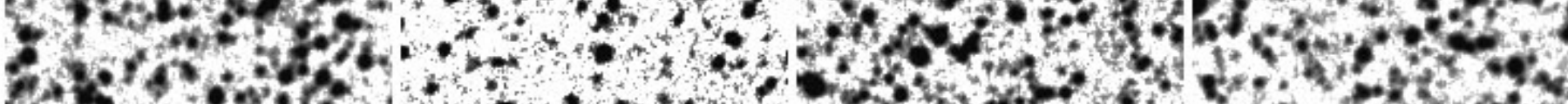

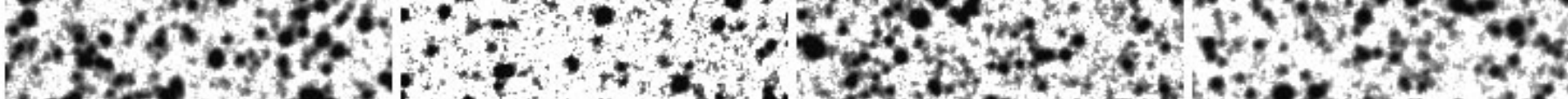

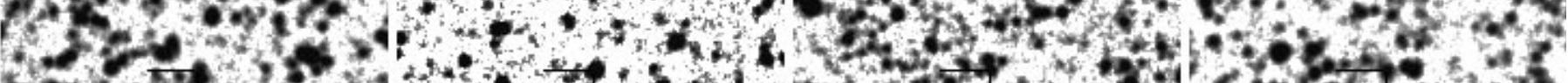

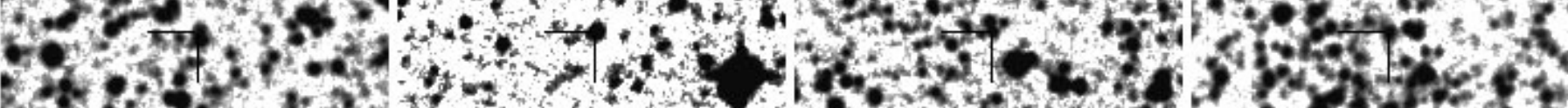

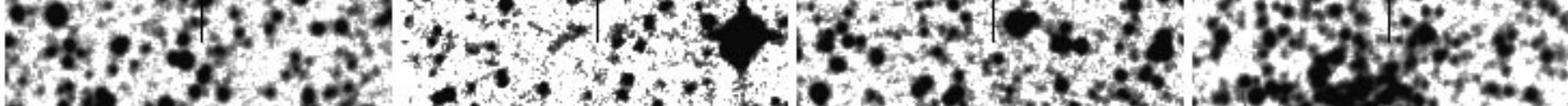

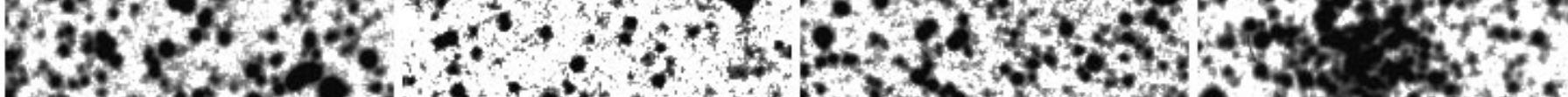

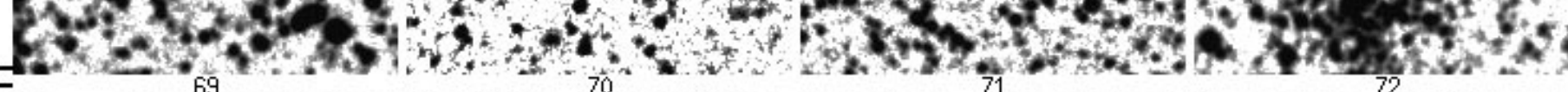

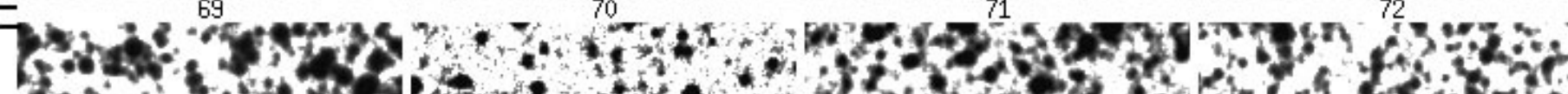

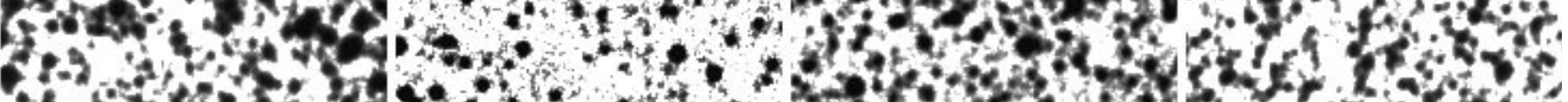

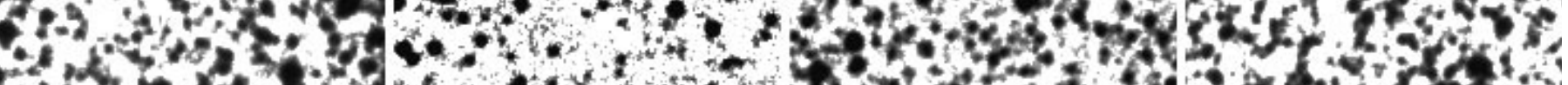

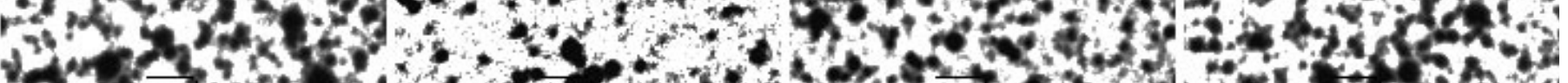
D.t.

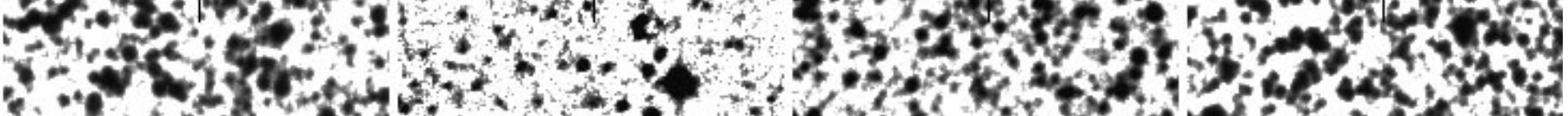

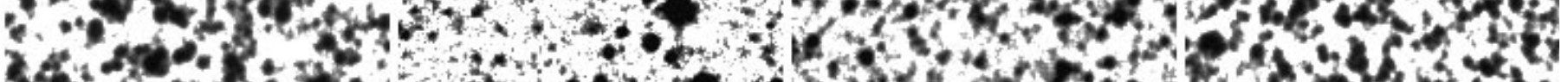

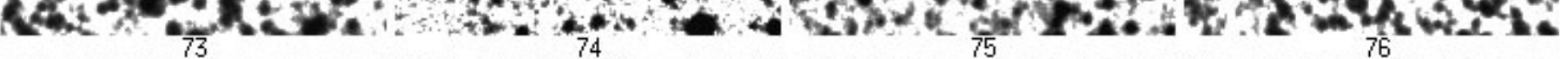

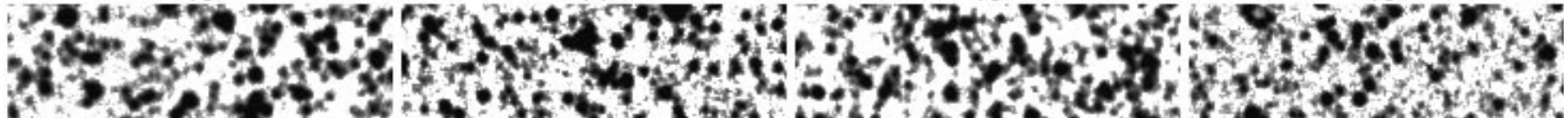

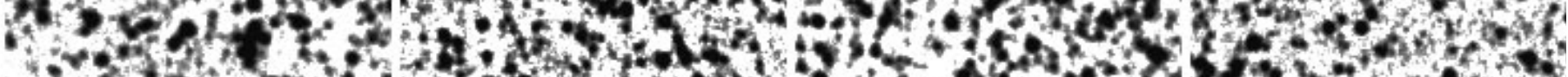

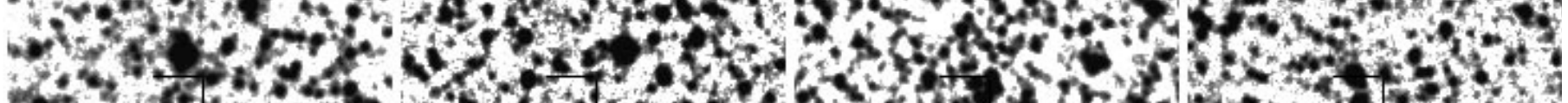

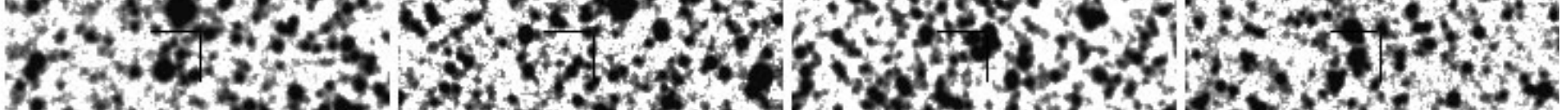

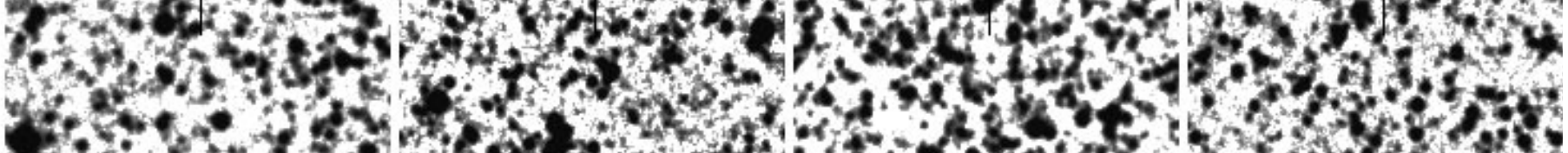
C. 


\section{$\mathbf{N}$}

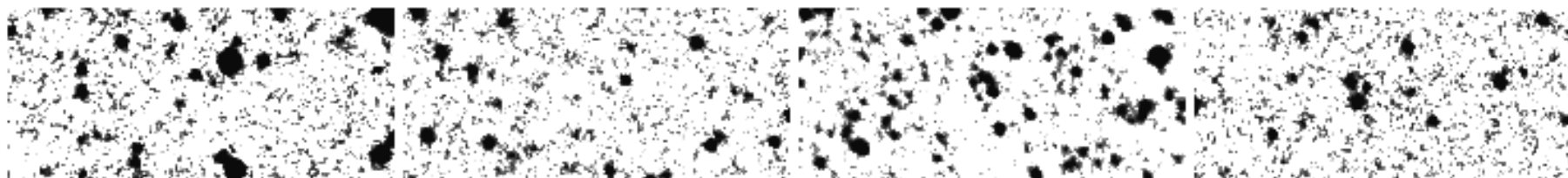

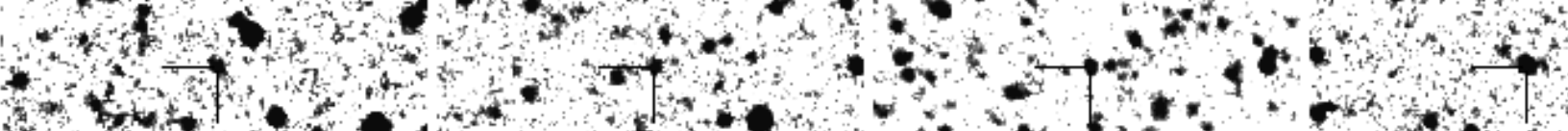

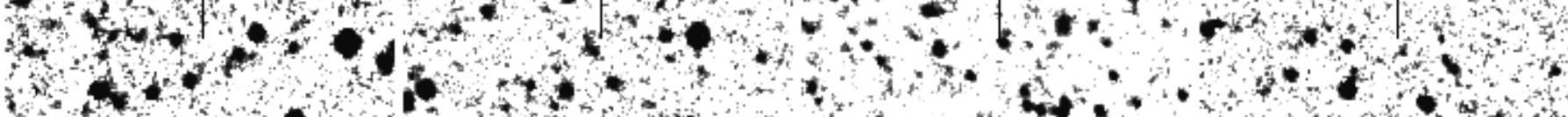

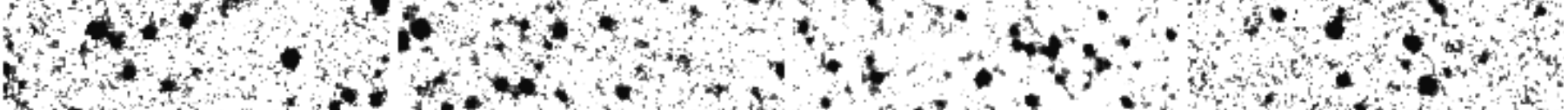

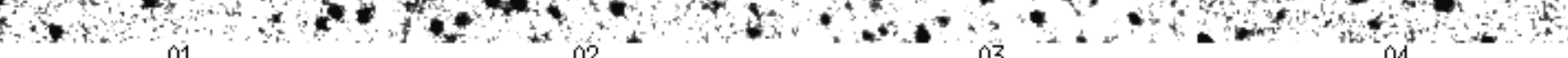

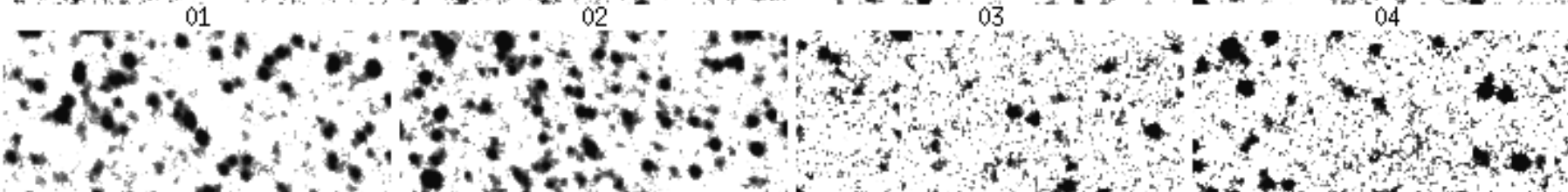

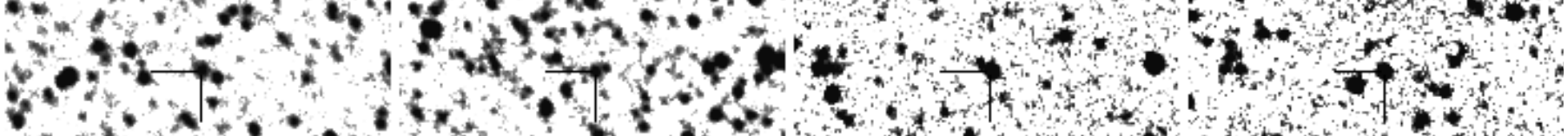

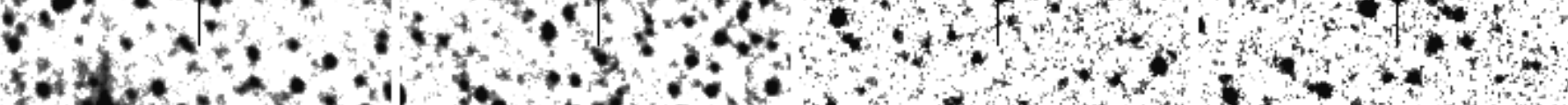

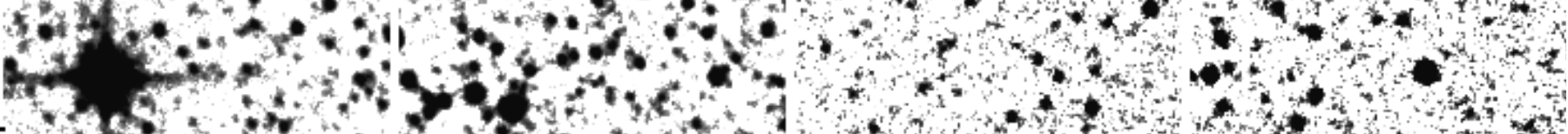

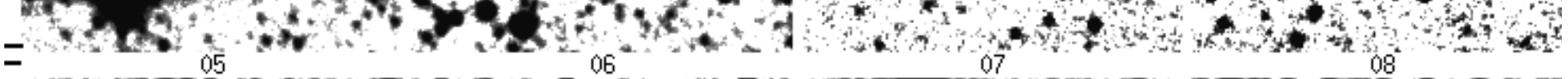

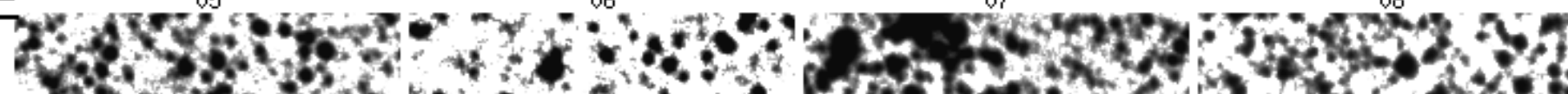

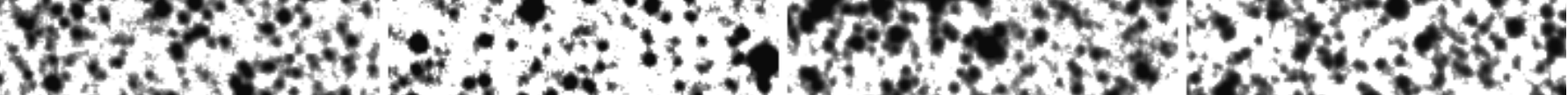

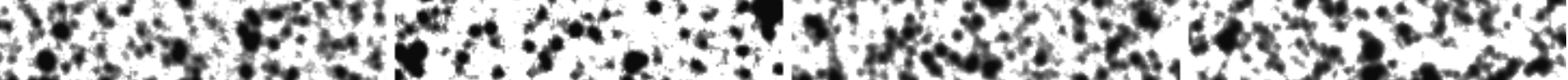

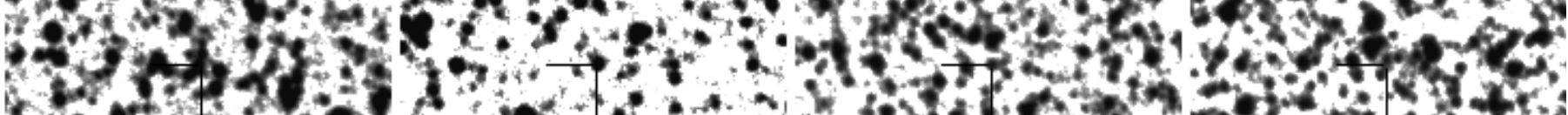

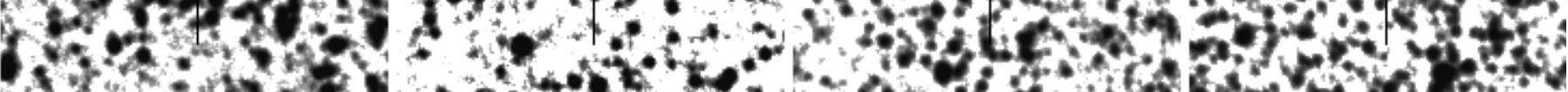

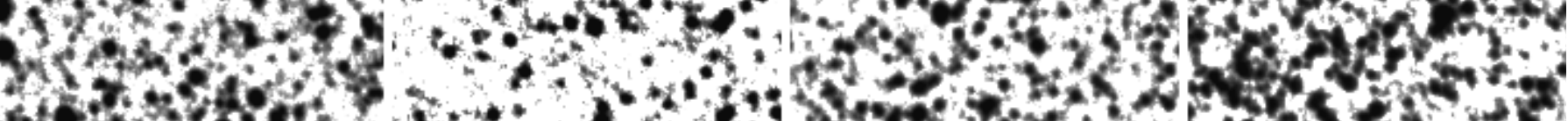

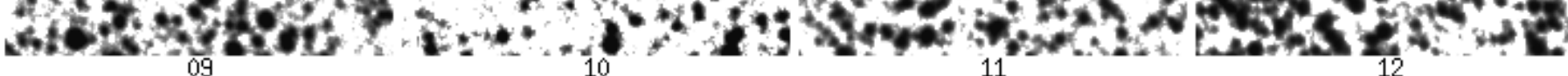

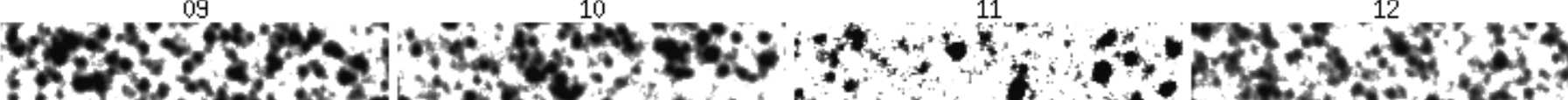

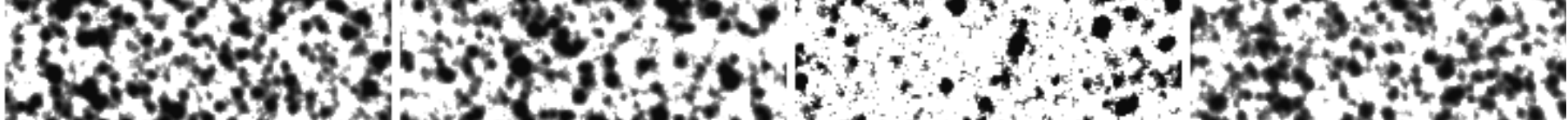

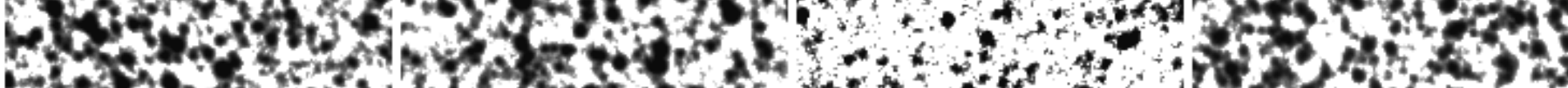

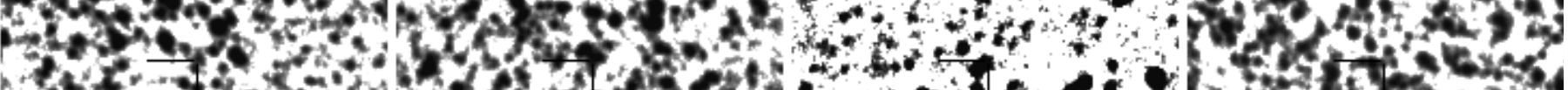

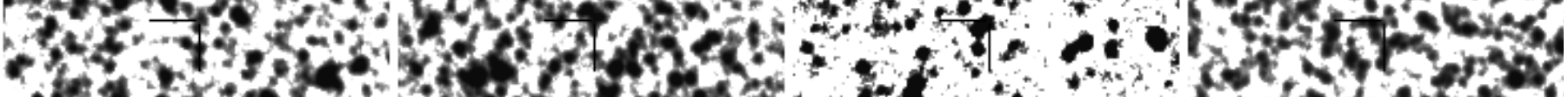

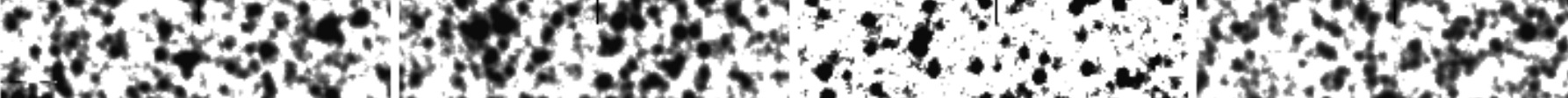

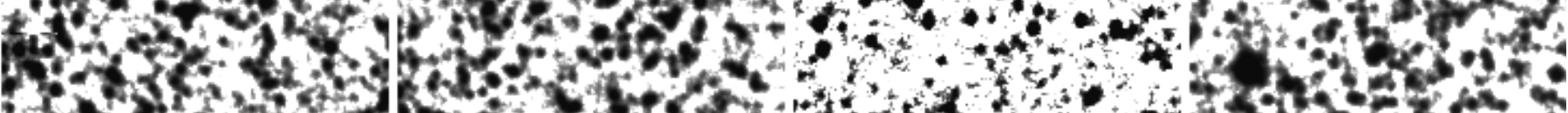

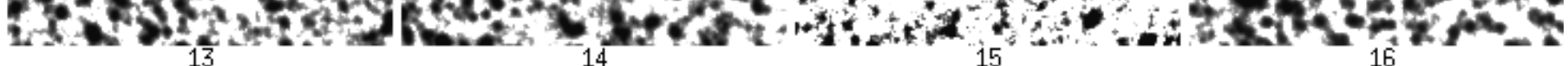


$\mathrm{N}$

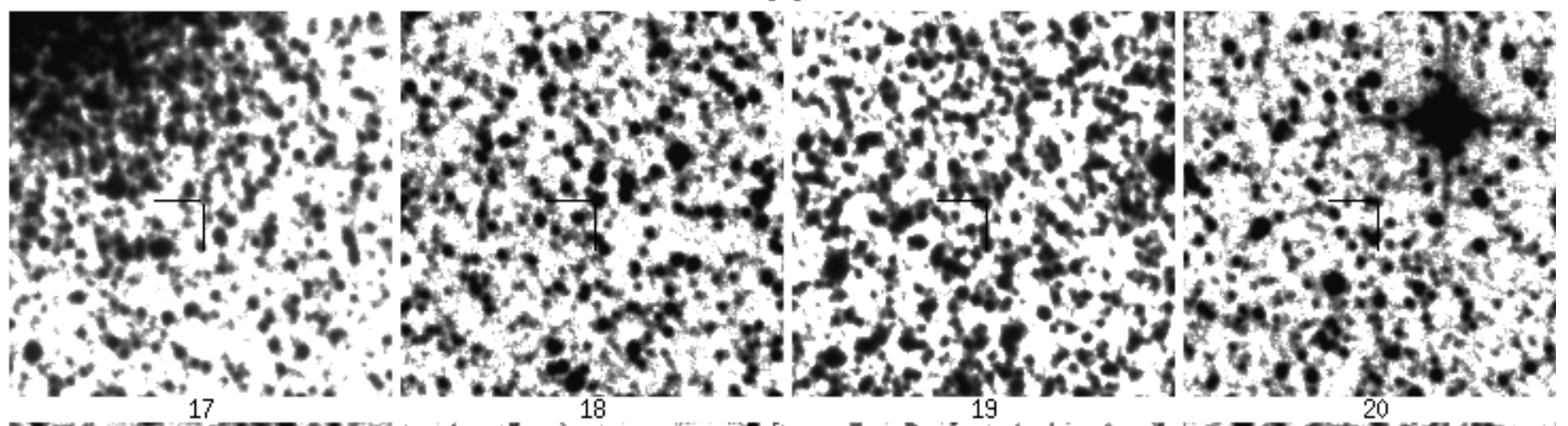

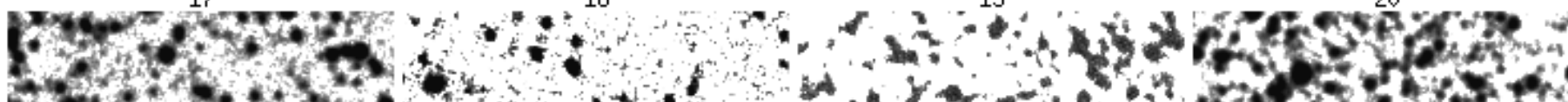

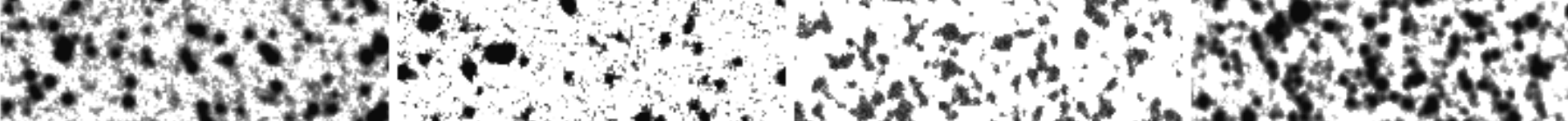

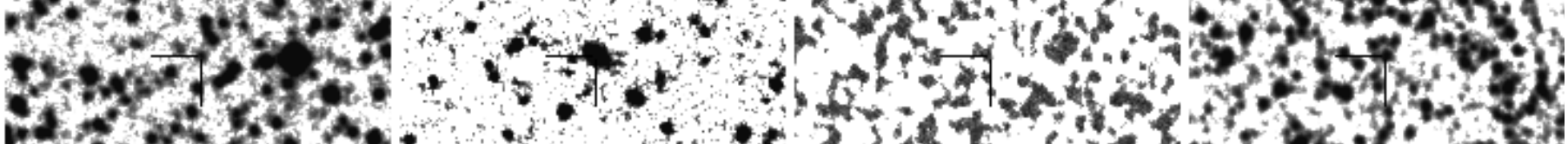

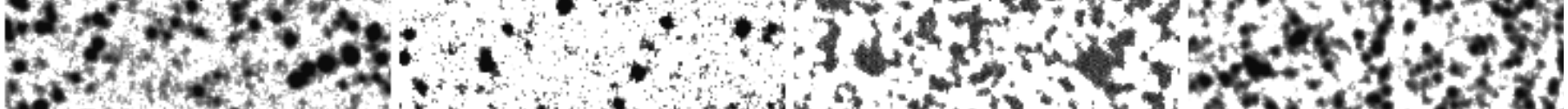

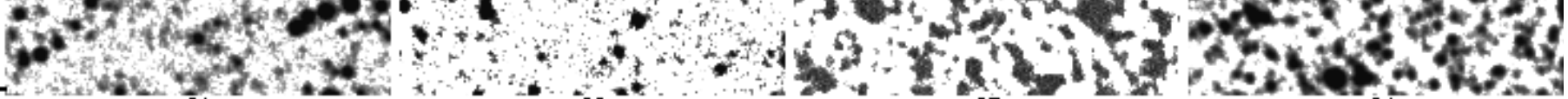

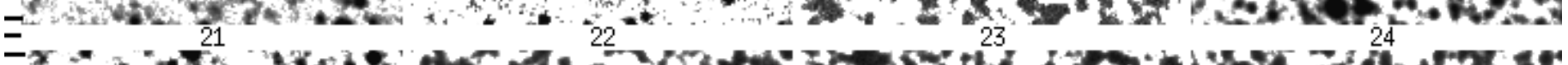
1.0.

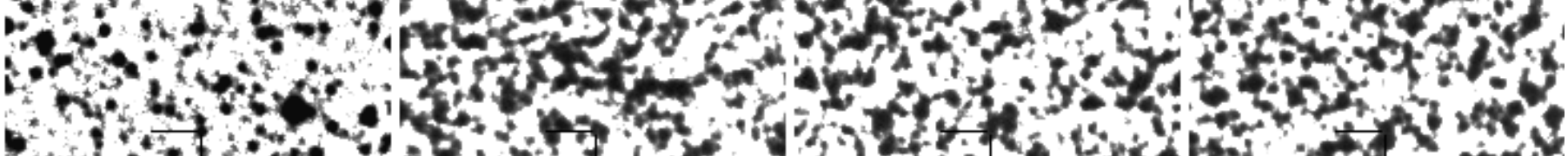
p.

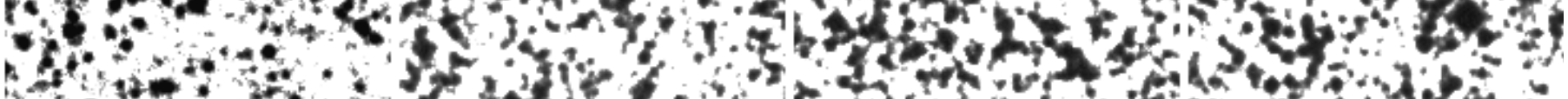

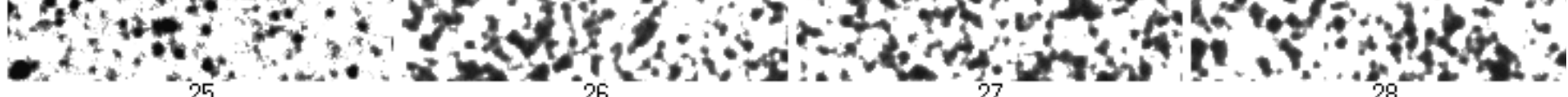

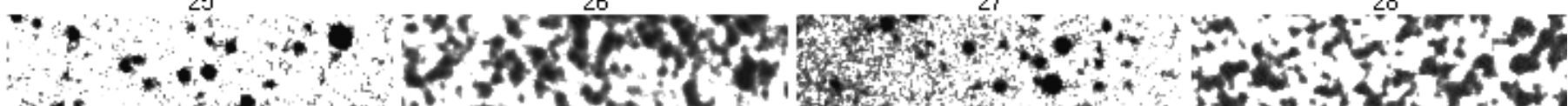

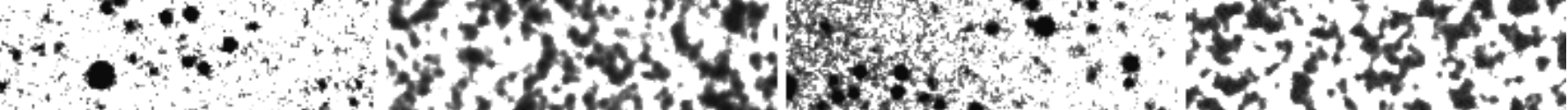

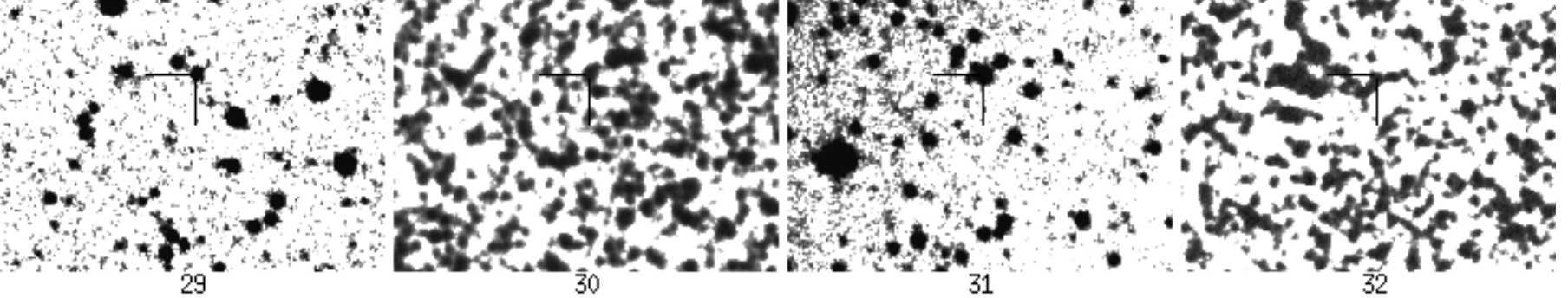


N

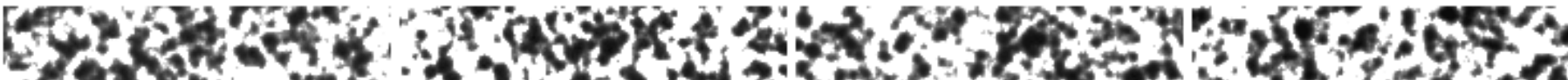

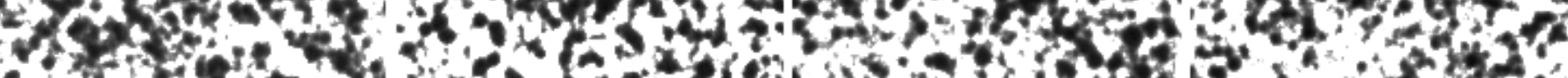

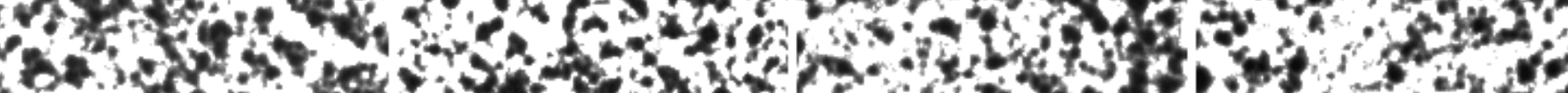

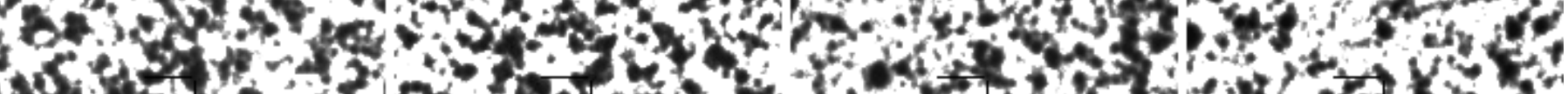

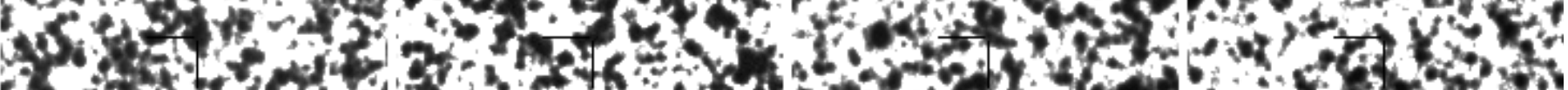
F.

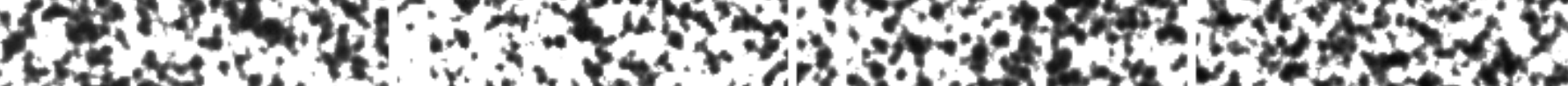

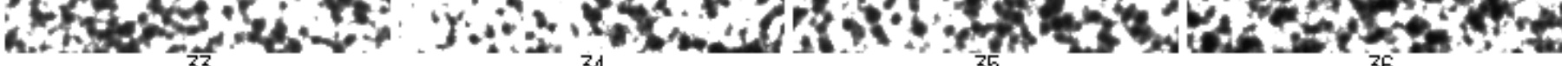

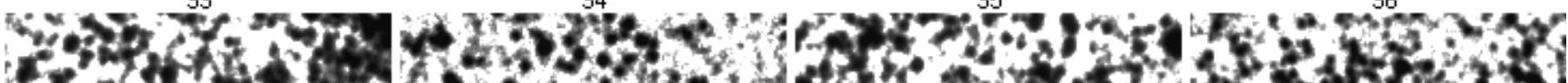

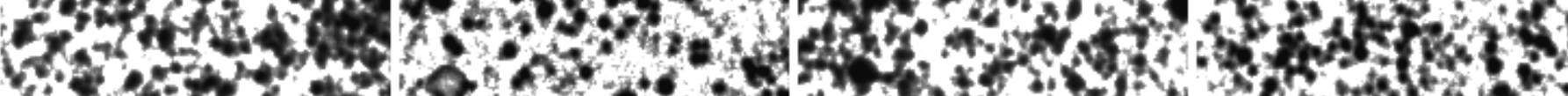

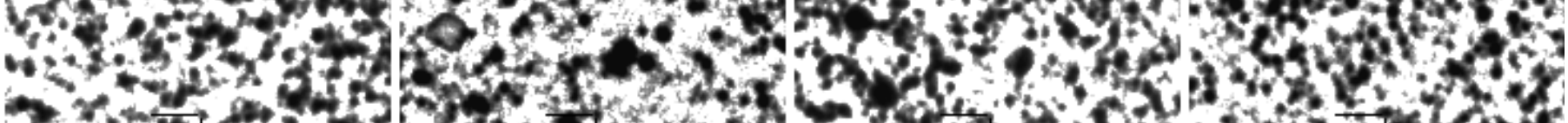

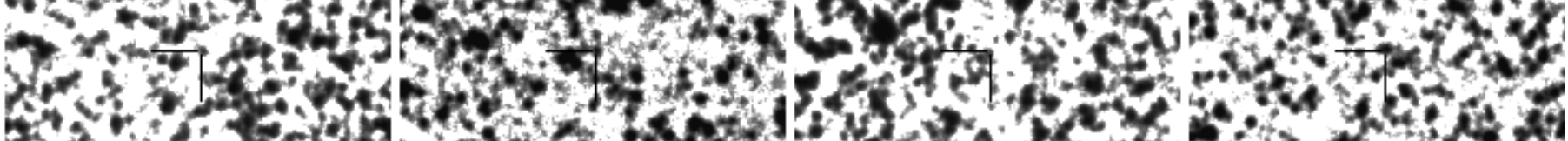

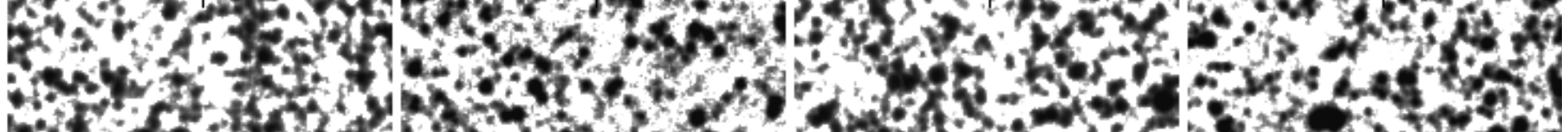

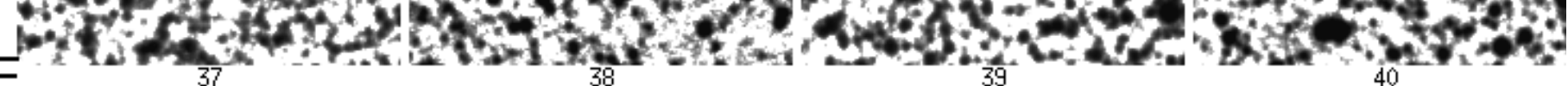

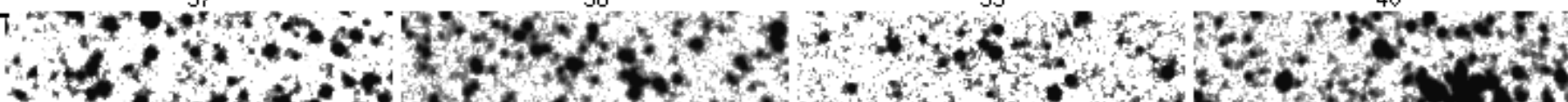

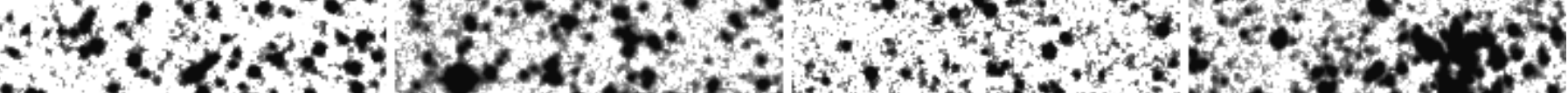

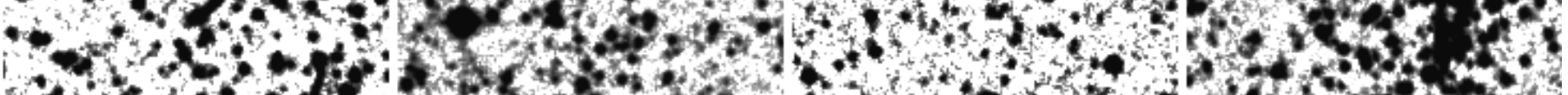

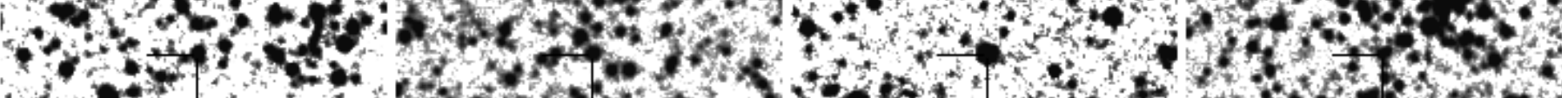

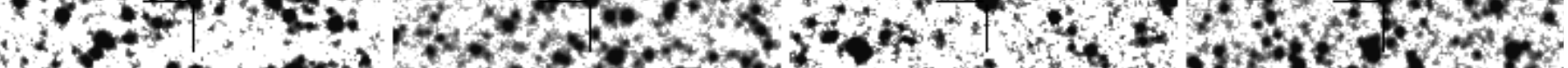

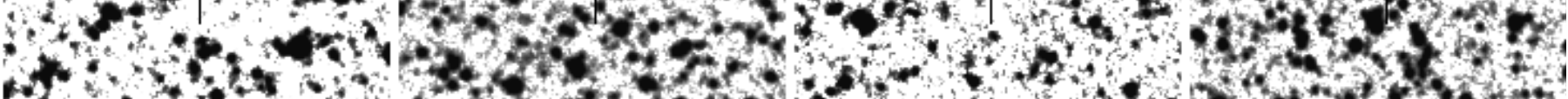

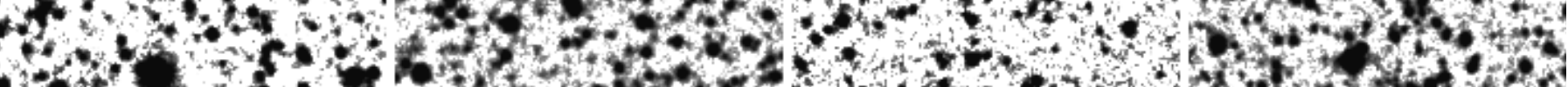

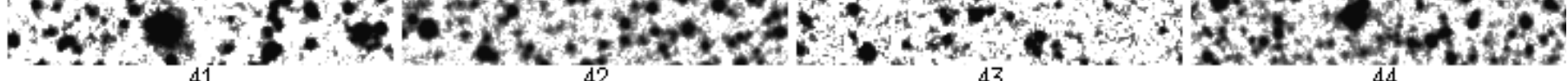

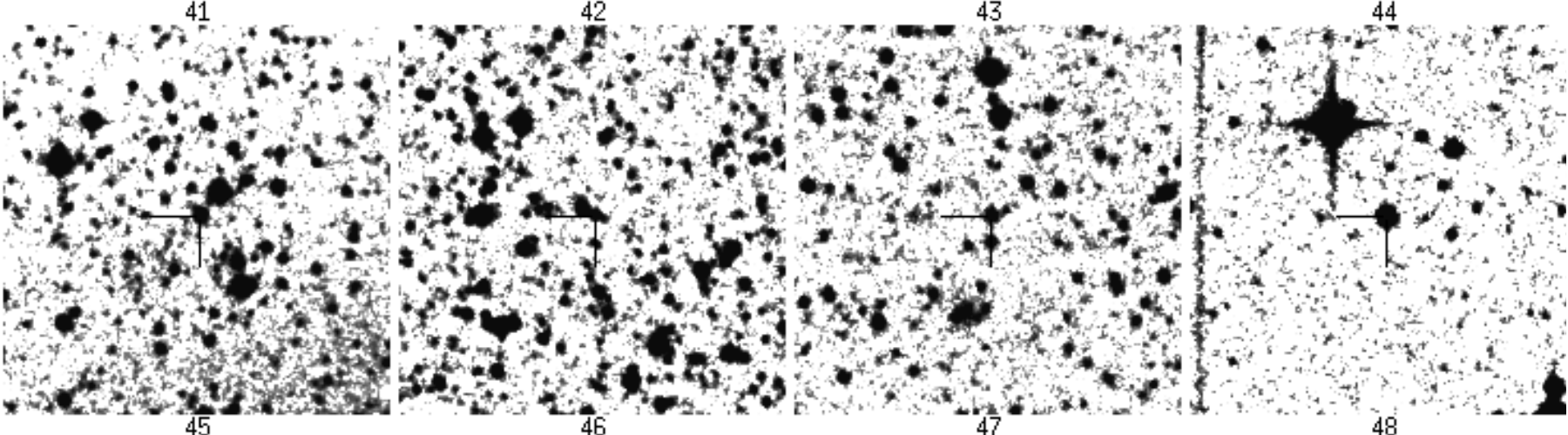




\section{$\mathbf{N}$}

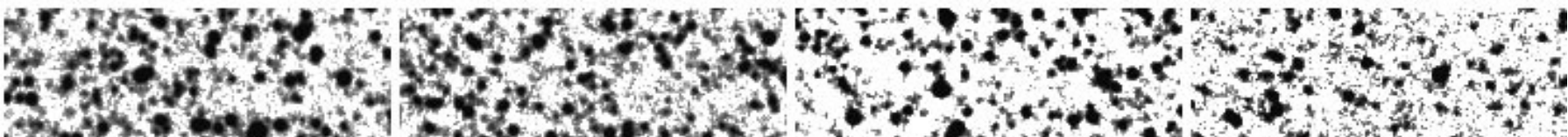

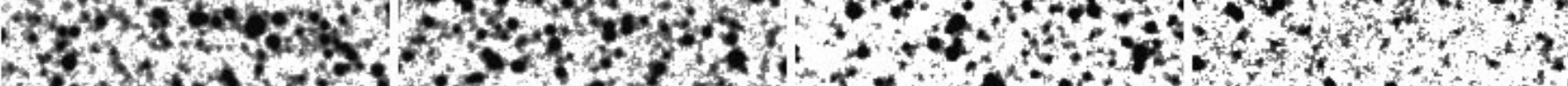

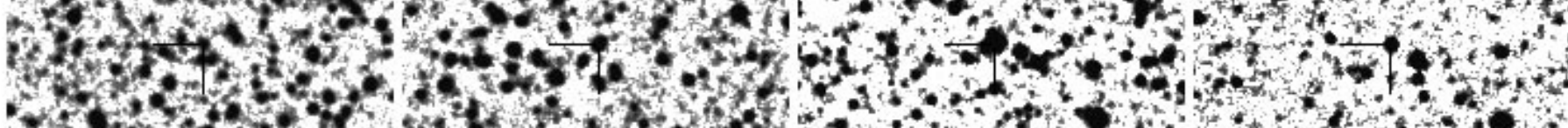

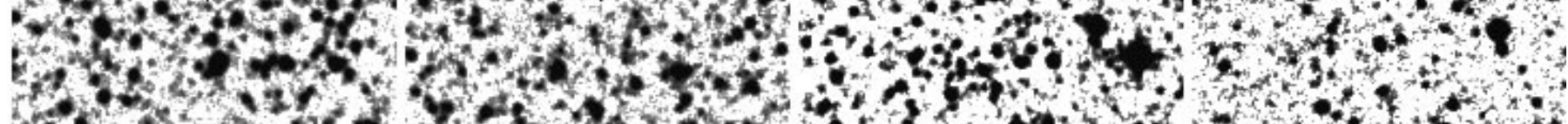

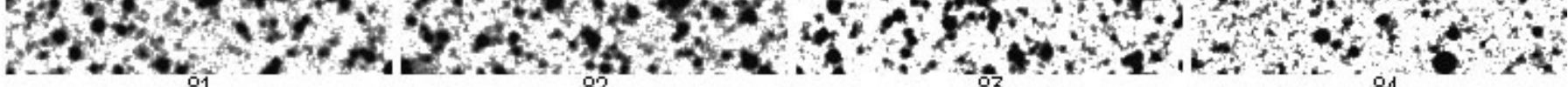

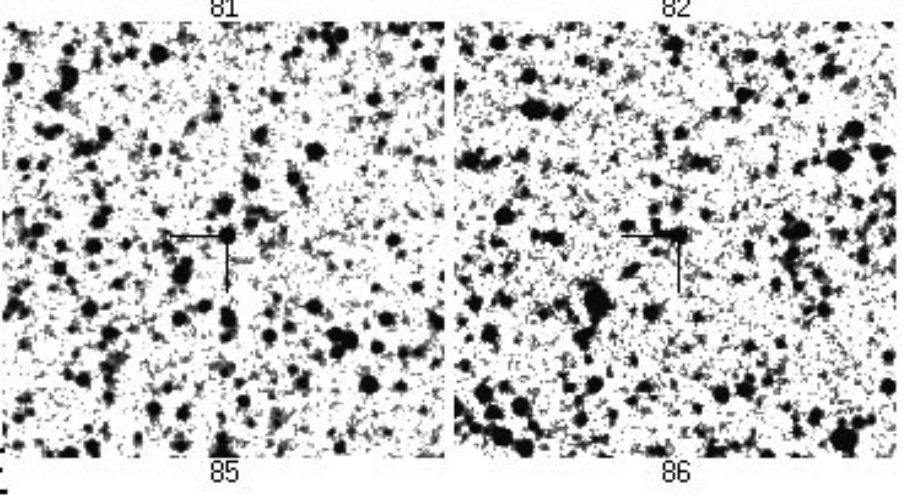

Fig. 20. Finding charts Morgan \& Good

\section{$\mathbf{N}$}
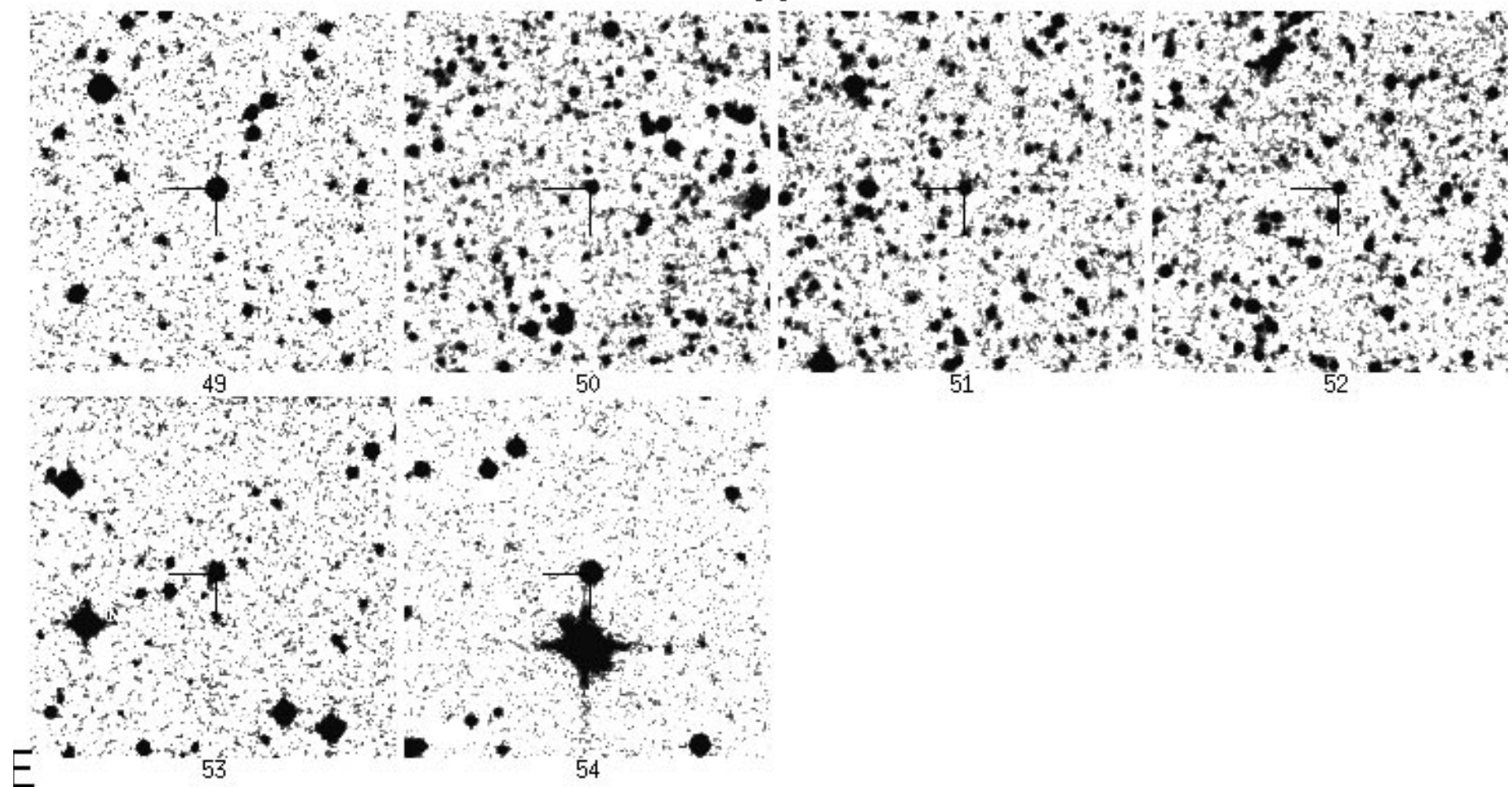

Fig. 24. Finding charts Morgan 